\title{
ANÁLISE ELASTODINÂMICA DE PLACAS ATRAVÉS DO MÉTODO DOS ELEMENTOS DE CONTORNO COM INTERAÇÃO SOLO-ESTRUTURA
}

\section{SAULO FARIA ALMEIDA BARRETTO}

Tese apresentada à Escola de Engenharia de São Carlos, da Universidade de São Paulo, como parte dos requisitos para obtenção do Título de Doutor em Engenharia de Estruturas.

ORIENTADOR: Prof. Dr: Wilson Sérgio Venturini

São Carlos

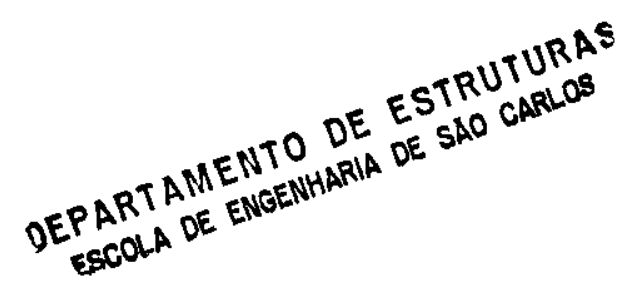

1995 


\section{Barretto, Saulo Faria Almeida}

B268a Análise elastodinâmica de placas, através do método dos elementos de contorno, com interação solo-estrutura / Saulo Faria Almeida Barretto. -- São Carlos, 1995. 130p.

Tese (Doutorado) -- Escola de Engenharia de São CarlosUniversidade de São Paulo, 1995.

Orientador: Prof.Dr. Wilson Sergio Venturini

1. Placas (Estruturas) - Análise elastostática. 2. Placas

(Estruturas) - Análise elastodinâmica. 3. Método dos elementos de contomo. 4. Interação solo-estrutura. I. Título. 
Aos meus pais,

Paulo e Dilma F. A. Barretto 


\section{AGRADECIMENTOS}

Ao Professor e amigo Wilson Sérgio Venturini, pela prestimosa orientaçāo e apoio dados a mim neste trabalho. Com certeza fica, desta etapa em que trabalhamos juntos, um respeito e uma admiração que me acompanharão para sempre.

Ao Professor e amigo Humberto Breves Coda, pela inestimável ajuda que me deu, colocando sempre a sua experiência e o seu conhecimento ao meu dispor. Da mesma forma, fica também um grande respeito e uma grande gratidão.

Ao Professor e amigo Heinz Antes, pela contribuição dada ao trabalho e pela forma como me recebeu na Alemanha, tornando a minha estadia nesse País não só mais agradável como também inesquecível.

Às pessoas abaixo relacionadas, que tiveram uma importante e também inesquecivel participação no desenvolvimento deste trabalho:

Renata Piazzalunga

Prof. Dr. João Batista de Paiva

Luttgardes de Oliveira Neto

João Ricardo Maia de Magalhães

Norberto Costardi 


\section{SUMÁRIO}

LISTA DE SIGLAS E SÍMBOLOS

RESUMO

ii

ABSTRACT

iii

1 INTRODUÇÃO

2 TEORIA BÁSICA, EQUAÇÕES INTEGRAIS E MÉTODO DOS ELEMENTOS DE CONTORNO APLICADOS A PLACAS

2.1 INTRODUÇÃO

2.2 EQUAÇÕES BÁSICAS 8

2.3 SOLUÇÃO FUNDAMENTAL DE PLACAS 16

2.4 EQUAÇŐES INTEGRAIS DE PLACAS 20

2.5 MÉTODO DOS ELEMENTOS DE CONTORNO

2.5.1 SOLUÇÃO DAS EQUAÇÕES INTEGRAIS 28

2.5.2 DEFINIÇÃO DO ELEMENTO 32

2.5.3 EQUAÇÕES MATRICIAIS PARA O ELEMENTO QUADRÁTICO 35

2.5.4 MONTAGEM DAS MATRIZES $H$ E $G$

2.5.5 INTEGRAL DE DOMINIO PARA MONTAGEM DO VETOR $F \quad 40$

2.5.6 CONDIÇÕES DE CONTORNO 42

2.5.7 MONTAGEM E SOLUÇĀO DO SISTEMA FINAL 43

2.6 EXEMPLOS NUMÉRICOS 47 
3 MÉTODO DOS ELEMENTOS DE CONTORNO APLICADOS

A PLACAS

3.1 INTRODUÇÃO 50

3.2 EQUAÇÃO INTEGRAL ELASTODINÂMICA

3.3. MONTAGEM DA MATRIZ DE MASSA $M$

3.4 SOLUÇĀO DO SISTEMA DINÂMICO 56

3.5 EXEMPLOS NUMÉRICOS 58

4 ANÁlISE DA ASSOCIAÇÃO PLACA-SOLO, APLICANDO EM AMBOS A FORMULAÇÃO DO MEC

4.1 INTRODUÇÃO 68

4.2 TRATAMENTO DO MEIO TRIDIMENSIONAL (SOLO) 69

4.2.1 SOLUÇÕES FUNDAMENTAIS

4.2.2 EQUAÇÕES INTEGRAIS 78

4.2.3 ASPECTOS NUMÉRICOS $\quad 77$

4.3 ACOPLAMENTO PLACA-SOLO 81

4.4 EXEMPLOS NUMÉRICOS 83

5 ANÁLISE DO COMPORTAMENTO ELASTODINÂMICO DE PLACAS UTILIZANDO-SE SOLUÇÃO FUNDAMENTAL TRANSIENTE, NO DOMINIO DO TEMPO

5.1 INTRODUÇÃO 94

5.2 EQUAÇÃO INTEGRAL TRANSIENTE DA PLACA 95

5.3 SOLUÇÃO FUNDAMENTAL NO DOMINIO DO TEMPO

5.3.1 EQUAÇÃO DIFERENCIAL TRANSFORMADA 99

5.3.2 SOLUÇÃO DA EQUAÇÃO INTEGRAL TRANSFORMADA 101

5.4 EQUAÇÃO INTEGRAL ESCRITA PARA OS PONTOS DE 103 CARREGAMENTO

5.5 INTEGRAÇĀO NO TEMPO

5.5.1 CONVOLUÇÃO DAS INTEGRAIS 105 
5.5.2 INTERVALOS DE INTEGRAÇÃO

106

5.6 MONTAGEM E RESOLUÇÃO DO SISTEMA

5.7 EXEMPLO NUMÉRICO

6 CONCLUSÕES

BIBLIOGRAFIA

APÊNDICE A 
$\pi=2 .{ }^{\star} \operatorname{ATAN2}(1,0$.

$\gamma=0.57721566 . . \quad$ (constante de Euler)

$x, y$ - eixo de coordenadas

$\mathrm{n}, \mathrm{s}$ - eixo de coordenadas

$\xi, \eta$ - eixo de coordenadas isoparamétricas

$\sigma$ - tensāo normal

$\tau$ - tensão de cisalhamento

$\varepsilon$ - deformação

$\gamma$ - deformaçāo transversal

$U$ - energia de deformação

w* - solução fundamental da placa

m - momento fletor

$q$ - força cortante

$\mathrm{V}$ - força cortante equivalente

$\mathrm{R}$ - raio de curvatura

$g$ - carregamento transversal

$\beta$ - ângulo que o eixo $\mathbf{n}$ forma com o vetor $\boldsymbol{r}$

$\theta$ - ângulo que o vetor $r$ forma com o eixo $x$

$\alpha$ - ângulo que o eixo $n$ forma com o eixo $x$

$\eta_{x}, \eta_{y}$ - cossenos diretores com relaçāo aos eixos $x$ e $y$

$\delta_{\mathrm{ij}}$ - Delta de Kronecker

$\delta_{(q, p)}$ - função Delta de Dirac

$\Gamma$ - contorno de um corpo

$\Omega$ - domínio de um corpo 
In - logaritmo neperiano

$|J|$ - jacobiano

$\phi_{(1)}$ - funções interpoladoras

$v$ - coeficiente de Poisson

E - módulo de elasticidade longitudinal

$\mathrm{G}=\frac{E}{2(1+v)} \quad$ (módulo de elastcidade transversal)

$\lambda=\frac{2 G v}{1-2 v} \quad$ (constante de Lamé)

$\mathrm{D}=\frac{E \cdot h^{3}}{12\left(1-v^{2}\right.} \quad$ (rigidez à flexão)

$\Delta=\left\{\frac{\partial^{2}}{\partial x^{2}}+\frac{\partial^{2}}{\partial y^{2}}\right\} \quad$ (operador de Laplace)

$\mathrm{Si}(\mathrm{x})=\int_{0}^{x} \frac{\operatorname{sen} \xi}{\xi} d \xi \quad$ (integral de seno)

$\mathrm{Ci}(\mathrm{x})=\int_{x}^{\infty} \frac{\cos \xi}{\xi} d \xi \quad$ (integral de cosseno)

MDF - Método das Diferenças Finitas

MEF - Método dos Elementos Finitos

MEC - Método dos Elementos de Contorno

$\mathrm{NN}$ - número de nós do contorno

NTOT - número de variáveis do contorno (2*NN)

NCAN - número de cantos da placa

NVARN - NTOT + NCAN

$\mathrm{NNI}$ - número de pontos internos

NVTT - número total de variáveis (NVARN + NNI) 


\section{RESUMO}

BARRETTO, Saulo F. A. Análise elastodinâmica de placas, através do método dos elementos de contorno, com interação solo-estrutura. São Carlos, 1995. 115p. Tese (Doutorado) - Escola de Engenharia de São Carlos, Universidade de São Paulo.

A combinação do Método dos Elementos de Contorno e do Método dos Elementos Finitos é o procedimento usualmente empregado na análise da flexão de placas interagindo com o solo. Usando-se da associação de ambos os métodos pode-se tirar vantagens de cada um deles $e$, consequentemente, chegar a uma técnica methorada para tratar com problemas práticos. Contudo, a formulação do MEF não representa bem as tensōes e os esforços concentrados ao longo do contorno, que podem ocorrer devido à maior rigidez da placa quando comparada com o meio solo, como a formulação do MEC faz. Por isso, a flexão de placas sobre base elástica é aqui proposta utilizando-se apenas das formulaçōes do MEC, ou seja, tanto os problemas tridimensionais quanto os problemas de placas são tratados pela formulação de contorno para casos elastostáticos e elastodinâmicos. Duas diferentes formas de tratar problemas de flexão elastodinâmica de placas são discutidas, enfatizando possiveis instabilidades numéricas que as duas técnicas podem exibir. Finalmente, depois de propor a combinação dos problemas tridimensional e de placas, os resultados de exemplos numéricos apresentados mostram as vantagens e desvantagens da técnica proposta.

Palavras-chave: Placas (Estruturas) - Análise elastostática; Placas (Estruturas) - Análise elastodinâmica; Método dos Elementos de Contorno; Interação solo-estrutura. 


\section{ABSTRACT}

BARRETTO, Saulo F. A. Elastodynamic analysis of plates, using the Boundary Element Method, with soil-structure interaction. São Carlos, $1995.115 \mathrm{p}$. Tese (Doutorado) - Escola de Engenharia de São Carlos, Universidade de São Paulo.

The combination of the boundary element and the finite element methods is the usually employed procedure to analyse plates in the bending interacting with the supporting soil. By using the association of both methods one can take the advantage of each method and consequently reach an improved technique to deal with practical problems. However, the FEM formulation can not represent well the stress and effort concentrations along the boundary, that may occur due to the higher plate stiffness when compared with the soil media, as the BEM technique does. Therefore, the plate bending on elastic foundation is proposed here using only BEM formulations, i.e. both the three-dimensional and the plate problems are formulated by boundary formulations for the elastostatic and elastodynamic cases. Two different ways to deal with the elastodynamic plate bending problem are discussed, emphasizing possible numerical instabilities that those techniques may exhibit. Finally, after proposing the combination of the three-dimensional and plate problems, results of numerical examples presented to show the advantages and disadvantages of the proposed technique.

Keywords: Plates (Structures) - Elastostatic analysis; Plates (Structures) Elastodynamic analysis; Boundary Element Method; Soil-structure interaction. 


\section{CAPÍTULO I}

\section{INTRODUÇÃO}

O desenvolvimento dos chamados métodos numéricos deveu-se ao fato de que as soluções analíticas dos diversos problemas da engenharia sempre ficavam limitadas,já que para estas soluções eram adotadas hipóteses simplificadoras, restringindo, assim, a análise de problemas particulares. Assim sendo, com o intuito de ampliar o leque de problemas a serem resolvidos por uma mesma solução, surgiram os métodos aproximados e, consequentemente, os métodos numéricos. Com o aperfeiçoamento dos computadores, resultando na sua maior capacidade de memória e velocidade de operação, os métodos numéricos foram ganhando cada vez mais importância na engenharia estrutural.

O primeiro método numérico a surgir foi o Método das Diferenças Finitas (MDF) e, segundo TIMOSHENKO (1980), a sua primeira aplicação na elasticidade è devida a C. Runge, em 1908. Este método consiste em aplicar, ponto a ponto, o operador diferença sobre a equação diferencial. Com o surgimento e desenvolvimento dos computadores, a aplicação e pesquisa dos métodos numéricos foi crescendo e surgiu o Método dos Elementos Finitos 
Sua idéia consiste em subdividir o domínio em "elementos", os quais sāo definidos pelos seus "nós", e é nestes elementos que são assumidas aproximações.

Na história do MEC um passo importante para a sua formulação foi a demonstração do teorema da reciprocidade por BETTI (1872), o qual permitiu que se escrevessem as equações integrais partindo-se da aplicação de um carregamento chamado fundamental. Em se tratando do método, especificamente, as primeiras formulaçōes que surgiram foram apresentadas por KUPRADZE (1965) e por RIZZO (1967). A primeira utilizou a técnica indireta, onde as variáveis empregadas não eram as variáveis físicas do problema, enquanto a segunda formulou o método de forma direta.

Com respeito à formulação elastodinâmica, a primeira formulação usando a técnica direta foi escrita por CRUSE (1968) e por CRUSE e RIZZO (1968). Essa formulação empregava a transformada de Laplace na análise de problemas elastodinâmicos. Posteriormente surgiu uma forma mais versátil e facilitada para resolver estes problemas, introduzido por NARDINI e BREBBIA (1982) e (1983), empregando o conceito de matriz de massa. Essa formulação se destaca pela simplicidade e por poder ser aplicada a diversos tipos de problemas, mas tem o incoveniente de provocar um aumento do trabalho e das dimensōes computacionais, já que a presença da integral de domínio do termo inercial exige uma discretizaçāo de todo o domínio. Neste campo de pesquisa deve-se considerar os trabalhos de CODA e VENTURINI (1990), LOEFFLER (1988), ANTES (1988), ESTORF et alli (1992) e CARRER (1991).

No caso específico da análise de placas, a aplicação do MEC ainda tem pouco desenvolvimento, sobretudo no que se refere a formulações e soluções fundamentais. O trabalho que é considerado o marco inicial na análise de placas via MEC é o de JASWON et alli (1967). Posteriormente BÉZINE (1978) e STERN (1979) desenvolveram a formulação direta para os problemas 
de placas finitas, com quaisquer condições de contorno. BÉZINE é o pesquisador que mais tem se dedicado ao estudo de placas através do MEC. Dele podemos citar trabalhos como análise de flexāo de placas cujas condiçōes de contorno estão no domínio (1981), substituiçāo da segunda equação integral (produto da derivada do deslocamento) por uma equação alternativa, baseada nas propriedades de similaridade geométrica da placa (1981), análise da flambagem de placas levando em conta apenas as condições de contorno no dominio (1985), etc.

O estudo elastodinâmico transiente de placas tem como referência o trabalho de BÉZINE (1982), que apresenta uma solução fundamental dinâmica transiente, no domínio do tempo. Ele se baseou no trabalho de SNEDDON (1944), o qual apresenta diversas soluções analiticas para casos de placas circulares, com raios infinitos ou nāo.

Com relação ao acoplamento solo-estrutura, após o surgimento do Método dos Elementos de Contorno a pesquisa passou a se concentrar na combinação do MEC com o MEF, visando explorar as vantagens de cada um, na análise das diferentes regiöes. No caso o solo, por ser o meio de domínio ilimitado, é tratado via BEM enquanto que a estrutura é tratada via MEF. Nessa direção pode-se citar o trabalho de ZIENKIEWICZ (1977) e o de ATLURI e GRANNELL (1978) como sendo os precursores deste tipo de análise. Posteriormente outros trabalhos foram surgindo em torno da idéia, entre os quais se referencia aqui os de MITSUl et alli (1985), que se introduz um elemento de contato entre as subregiōes para facilitar a ligaçāo quando existir diferenças das constantes físicas das subregiōes, GANGMING (1989), que simetriza a matriz de rigidez obtida através do BEM, e MESSAFER (1989), que trata da ligação placa-solo. No caso de ligação solo-estrutura em que ambas as regiōes sejam tratadas via BEM, pode-se citar o trabalho apresentado por 
PAIVA e BUTTERFIELD (1995), que trata de análise elastostática de ligação placa-solo.

O objetivo deste trabalho consiste em utilizar o Método dos Elementos de Contorno (MEC) para a análise estática e dinâmica transiente de placas, e em apresentar o comportamento destas quando ligadas ao solo. Neste trabalho será apresentada a formulação elastostática e elastodinâmica da placa utilizando-se da mesma solução fundamental, independente da variável tempo, para em seguida tratar da ligação solo-estrutura.

A solução fundamental adotada é a apresentada por DANSON (1979) e o tratamento elastodinâmico das placas foi feito utilizando-se da forma alternativa, via "matriz de massa". Algum comentário sobre essa forma de se resolver um problema dinâmico já foi feito anteriormente. e exemplos de diferentes discretizaçōes do dominio, para o mesmo tipo de placas, são apresentados. A intenção desta comparação entre os exemplos é mostrar a eficiência e a versatilidade da formulação.

Uma outra formulação para a análise dinâmica de placas é apresentada a partir da solução fundamental do BÉZINE (1982). Com esta formulação, um exemplo de placa circular é analisado $e$, através dos resultados, pode-se observar os problemas de instabilidade que surgem. $A$ intenção que se teve em mostrar os problemas da formulação é a de provocar uma discussão em torno dos mesmos, na busca de caminhos para solucionálos.

Para a interaçāo solo-estrutura utilizou-se do programa numérico para análise tridimensional desenvolvido por CODA (1993), tanto para a análise elastostática quanto elastodinâmica. O acoplamento numérico dos dois programas foi feito através da técnica de subregiöes [VENTURINI (1983), LACHAT (1975a) e LACHAT (1975b)]. Esta técnica consiste em aplicar 
separadamente as equações correspondentes de cada região e acoplá-las, utilizando-se de condições de compatibilidade cinemática e de equilibrio.

\section{Descrição dos capítulos}

No capítulo II é feita uma revisão das equações básicas que regem o comportamento de placas. Esta revisão é voltada para as equaçōes que são diretamente utilizadas na formulação do MEC. Em seguida é apresentado o desenvolvimento da solução fundamental de placa que será utilizada tanto na análise elastostática quanto na elastodinâmica. Definida a solução fundamental, parte-se para a formulação das equações integrais. Finalmente a formulaçāo Método dos Elementos de Contorno para a determinação estática de placas é apresentada. Nele se define o tipo de elemento adotado, as técnicas de integraçāo do dominio, a montagem das equaçōes matriciais, a montagem das matrizes $H$ e $G$, bem como o vetor de carregamento $\boldsymbol{F}$. Em seguida, são apresentadas as condições de contorno e a solução do sistema. Finalmente emprega-se a formulaçăo na análise de placas retangulares.

No capítulo III é apresentada a formulação elastodinâmica de placas partindo-se do conceito de matriz de massa. A solução fundamental é a mesma do capítulo anterior e é desenvolvida a integração no domínio do termo inercial, a partir do tipo de célula adotada. Uma vez obtida a matriz de massa, o sistema é resolvido através do método de Newmark [ WARBURTON (1964) ]. Os mesmos exemplos de placas analisados no capítulo II são também tratados neste capítulo, agora em sua versão dinâmica, variando-se apenas a discretização do domínio. 
O capitulo IV trata da ligação solo-estrutura. Inicialmente apresenta-se a formulação empregada para tratar do solo e, em seguida, demonstra-se de que forma o acoplamento foi obtido para, finalmente, serem apresentados os exemplos numéricos, comparados com soluções já obtidas em outras referências.

No capítulo $\mathrm{V}$ apresenta-se o desenvolvimento empregado por Bézine na obtenção de sua solução fundamental transiente de placas bidimensionais. Neste capítulo se discute os problemas de estabilização que a solução apresenta.

No capítulo VI são feitas as considerações gerais sobre o trabalho desenvolvido e apresentadas sugestōes no sentido de aperfeiçoar os resultados obtidos. 
TEORIA BÁSICA, EQUAÇÕES INTEGRAIS E MÉTODO DOS ELEMENTOS DE CONTORNO APLICADOS A PLACAS

- ELASTOSTÁTICA -

\section{1 - Introdução}

Como sabemos, placas são elementos estruturais de superfície definidos por dois planos paralelos (que podem ter pequena curvatura) e distantes entre si da espessura que os define. Essa espessura tem dimensão sempre pequena, se comparada com as outras duas.

No presente trabalho somente serāo consideradas as placas delgadas, isótropas ( com propriedades iguais em todas as direções), com carregamento aplicado sempre na direção transversal ao plano médio e que obedeçam a teoria de pequenos deslocamentos.

Dentro dessa especificidade valem as seguintes hipóteses simplificadoras: 
1. o material da placa é elasto-linear;

2. os deslocamentos transversais ao plano médio são pequenos;

3. as deformações do plano médio são consideradas desprezíveis;

4. as tensōes normais na direção transversal são consideradas despreziveis;

5. as seções planas, perpendiculares à superfície média antes do carregamento continuam planas, após o carregamento.

\section{2 - Equações básicas}

Considerando-se que as deduçōes que nos levam às equações básicas, as quais regem o comportamento de placas, estão amplamente repetidas em várias referências ( PAIVA (1990), OLIVEIRA NETO (1991), etc.) optou-se aqui por abstrai-las. Assim sendo, apenas as expressões que entram nas equações integrais do método serão descritas, e de forma a mais suscinta possível. Para um acompanhamento mais detalhado do que será apresentado a seguir, recomenda-se TIMOSHENKO (1970).

Convém lembrar, inicialmente, que o comportamento elasto-linear da placa é regido pelas seguintes equações:

- relação tensão-deformação, obtidas a partir da Lei de Hooke

$$
\sigma_{x x}=\frac{E}{1-v^{2}}\left(\varepsilon_{x x}+v \varepsilon_{y y}\right)
$$




$$
\begin{aligned}
& \sigma_{y y}=\frac{E}{1-v^{2}}\left(\varepsilon_{y y}+v \varepsilon_{x x}\right) \\
& \tau_{x y}=G \gamma_{x y}
\end{aligned}
$$

- relação deformaçāo-deslocamento (observando-se a teoria dos pequenos deslocamentos)

$$
\begin{aligned}
& \varepsilon_{x x}=-z \frac{\partial^{2} w}{\partial x^{2}} \\
& \varepsilon_{y y}=-z \frac{\partial^{2} w}{\partial y^{2}} \\
& \gamma_{x y}=-2 z \frac{\partial^{2} w}{\partial x \partial y}
\end{aligned}
$$

As equações integrais do MEC, como será visto mais adiante, estão expressas em função do deslocamento transversal, da rotação, da força cortante e do momento fletor, estando as expressōes desses três últimos parâmetros em função do primeiro. Assim sendo, torna-se necessário obter estas expressões, escritas em função do deslocamento $\boldsymbol{w}$. Para tanto deve-se analisar, inicialmente, o equilíbrio de momentos e forças cortantes em um elemento infinitesimal de placa. A partir desse equilíbrio escrevem-se as expressões dos momentos e forças cortantes das placas, em função de $w$.

Isto pode ser feito representando-se os esforços que atuam no elemento de placa através de suas resultantes aplicadas na superficia média do mesmo, como nas figuras 01 e 02 a seguir. Considerando-se o carregamento transversal $(g)$ que atua no sentido positivo do eixo $z$, 


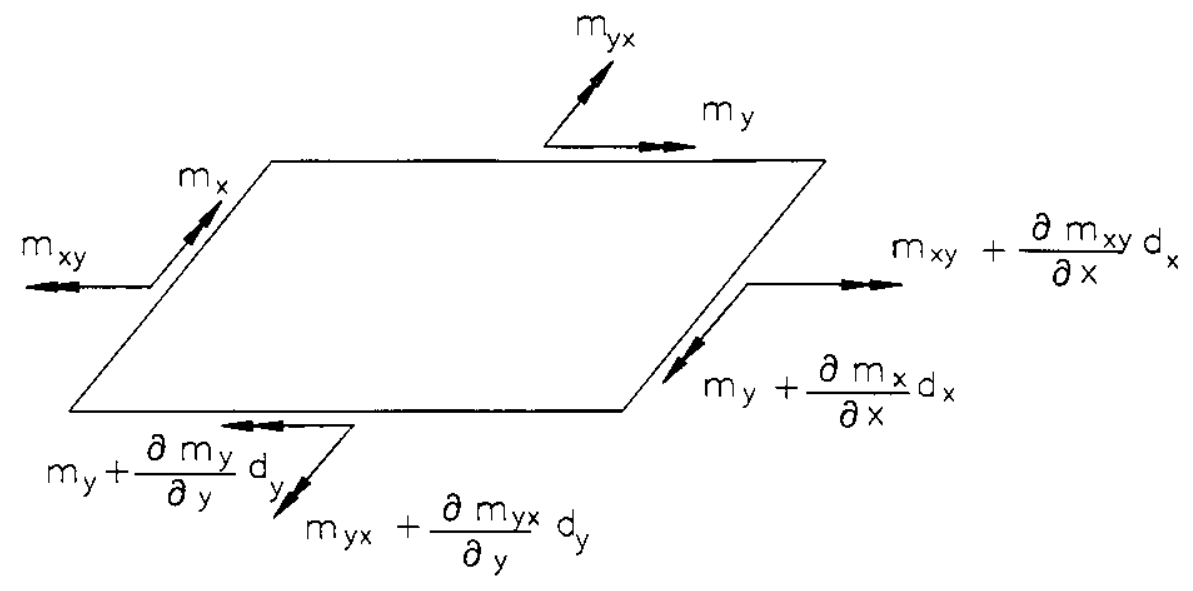

FIGURA 01

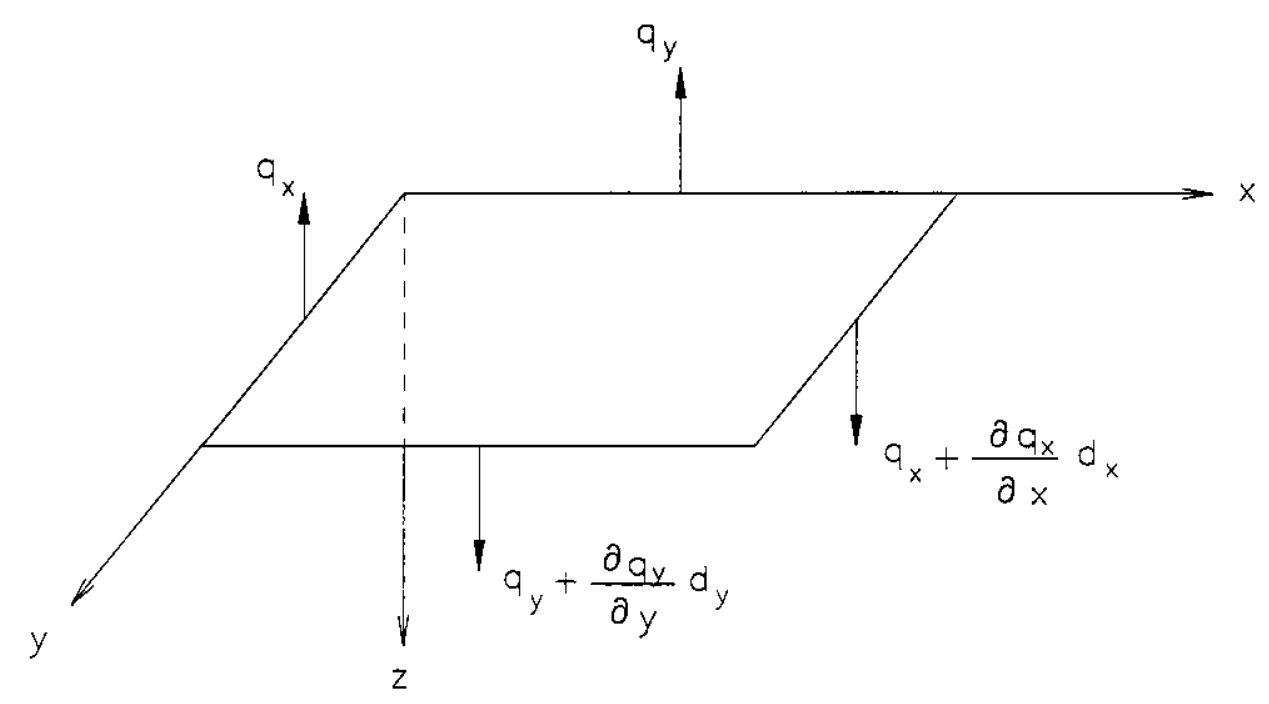

FIGURA 02

obtém-se a equação de equilíbrio da placa, as expressões dos momentos e forças cortantes, assim como a equação de equilíbrio em função do deslocamento $w$. 


$$
\begin{aligned}
& \frac{\partial^{2} m_{x}}{\partial x^{2}}+\frac{\partial^{2} m_{y}}{\partial y^{2}}+2 \frac{\partial^{2} m_{x y}}{\partial x \partial y}=-g \\
& m_{x}=-D\left(\frac{\partial^{2} w}{\partial x^{2}}+v \frac{\partial^{2} w}{\partial y^{2}}\right) \\
& m_{y}=-D\left(\frac{\partial^{2} w}{\partial y^{2}}+v \frac{\partial^{2} w}{\partial x^{2}}\right) \\
& m_{x y}=m_{y x}=-D(1-v) \frac{\partial^{2} w}{\partial x \partial y} \\
& q_{x}=-D \frac{\partial}{\partial x}\left(\frac{\partial^{2} w}{\partial x^{2}}+\frac{\partial^{2} w}{\partial y^{2}}\right) \\
& q_{y}=-D \frac{\partial}{\partial y}\left(\frac{\partial^{2} w}{\partial y^{2}}+\frac{\partial^{2} w}{\partial x^{2}}\right)
\end{aligned}
$$

e, finalmente, substituindo-se II.4, II.5 e II.6 em II.3, obtém-se a expressão diferencial da placa em função do deslocamento transversal $\boldsymbol{w}$.

$$
\frac{\partial^{4} w}{\partial x^{4}}+2 \frac{\partial^{4} w}{\partial x^{2} \partial y^{2}}+\frac{\partial^{4} w}{\partial y^{4}}=\frac{g}{D}
$$


Quando se pretende analisar um problema qualquer, torna-se conveniente reescrever as expressões anteriores, deixando-as referidas não mais aos eixos cartesianos $\mathrm{x}$ e y, mas a um eixo genérico de coordenadas $n$ e $s$, o qual forma um ângulo $\alpha$ com semi-eixo positivo $x$. Para isto, deve-se desenhar o equilíbrio em um novo elemento infinitesimal de placa, levando-se em conta o novo eixo de coordenadas. Desta forma, pode-se obter as relações geométricas que refrenciam um eixo ao outro.

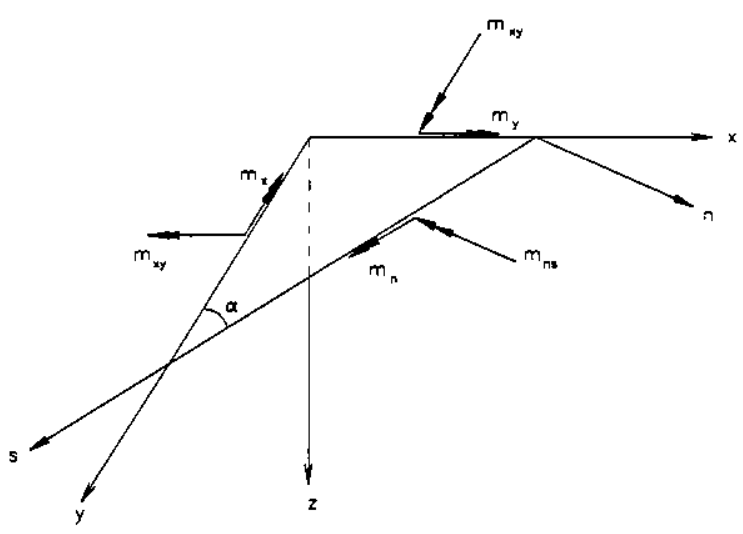

FIGURA 03

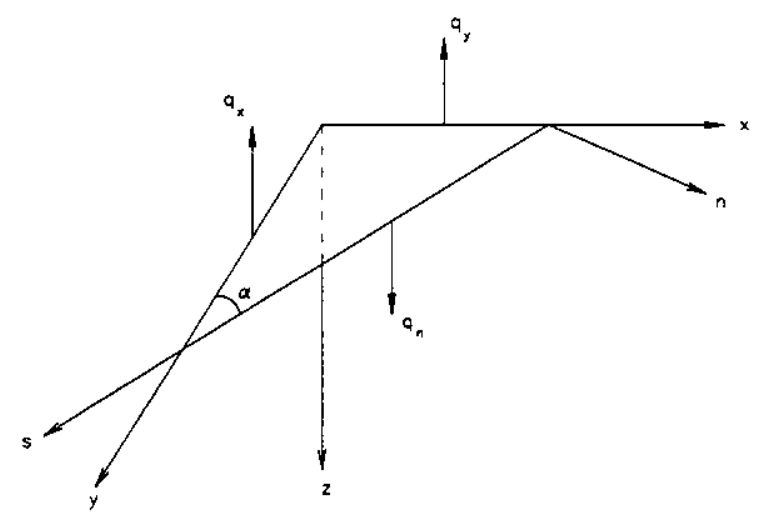

FIGURA 04 
Fazendo-se novamente 0 equilíbrio do elemento, pode-se escrever as expressões de $\boldsymbol{m}_{n}, \boldsymbol{m}_{n s}, \boldsymbol{q}_{n} \in \boldsymbol{q}_{s}$ em função dos momentos e cortantes referidos ao sistema global e do ângulo $\alpha$.

$$
\begin{aligned}
& m_{n}=m_{x} \cos ^{2} \alpha+m_{y} \operatorname{sen}^{2} \alpha+2 m_{x y} \operatorname{sen} \alpha \cos \alpha \\
& m_{n s}=\left(m_{y}-m_{x}\right) \operatorname{sen} \alpha \cos \alpha+m_{x y}\left(\cos ^{2} \alpha-\operatorname{sen}^{2} \alpha\right) \\
& q_{n}=q_{x} \cos \alpha+q_{y} \operatorname{sen} \alpha
\end{aligned}
$$

Quando se pretende resolver uma equação diferencial da placa, faz-se necessário a imposição de condições de contorno. No caso, essas condições podem ser o deslocamento tranversal $w$, a rotação normal $\partial w / \partial n$ e os esforços $m_{n}, m_{n s}$ e $q_{n}$. Sendo a equação diferencial de $4^{\text {a }}$ ordem, exeistem 4 valores de contorno que devem ser associados a cada ponto. Assim, apenas dois dos esforços podem ser considerados. KIRCHHOFF (1850) demonstrou que as duas últimas condições de contorno podem ser agrupadas em uma única, denominada de força cortante equivalente, cuja expressão é:

$$
V_{n}=q_{n}+\frac{\partial m_{n s}}{\partial s}
$$

Finalmente, como as equações integrais do Método dos Elementos de Contorno estāo escritas em coordenadas polares, torna-se útil reescrever as expressões anteriores, referentes aos eixos $n$ e $s$, deixando-as em coordenadas polares. 


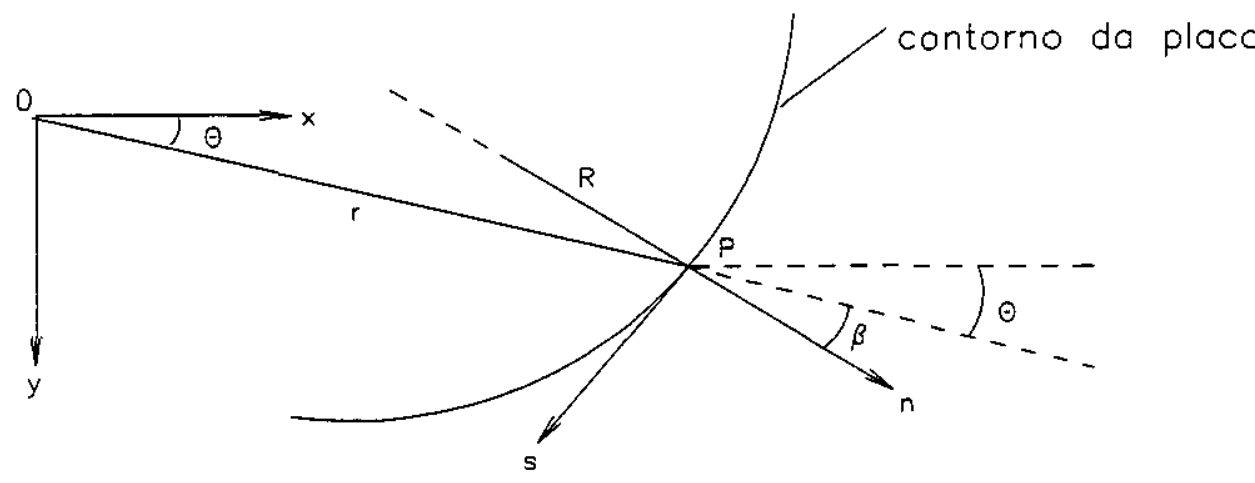

$\alpha=\Theta+\beta$

FIGURA 05

$$
\begin{aligned}
& x=r \cos \theta \\
& y=r \operatorname{sen} \theta \\
& r^{2}=x^{2}+y^{2} \\
& \theta=\operatorname{arctg} \frac{y}{x}
\end{aligned}
$$

De posse destas relações, as expressões (II.10), (II.11), (II.12), (II.13) e a equação diferencial da placa (II.9) podem ser escritas em coordenadas polares.

$\frac{\partial w}{\partial n}=\frac{\partial w}{\partial r} \cos \beta$ 


$$
\begin{aligned}
m_{n}= & -D\left[\left(\cos ^{2} \beta+v \operatorname{sen}^{2} \beta\right) \frac{\partial^{2} w}{\partial r^{2}}+\left(\operatorname{sen}^{2} \beta+v \cos ^{2} \beta\right)\left(\frac{1}{r} \frac{\partial w}{\partial r}+\frac{1}{r^{2}} \frac{\partial^{2} w}{\partial \theta^{2}}\right)\right. \\
& \left.+2(1-v) \operatorname{sen} \beta \cos \beta \frac{\partial}{\partial r}\left(\frac{1}{r} \frac{\partial w}{\partial \theta}\right)\right] \\
m_{n s}= & -D(1-v)\left[\operatorname{sen} \beta \cos \beta\left(\frac{1}{r} \frac{\partial w}{\partial r}+\frac{1}{r^{2}} \frac{\partial^{2} w}{\partial \theta^{2}}-\frac{\partial^{2} w}{\partial r^{2}}\right)+\left(\cos ^{2} \beta-\operatorname{sen}^{2} \beta\right)\right. \\
& \left.\frac{\partial}{\partial r}\left(\frac{1}{r} \frac{\partial w}{\partial \theta}\right)\right] \\
q_{n}= & -D\left(\frac{\partial}{\partial r} \Delta w \cos \beta+\frac{1}{r} \frac{\partial}{\partial \theta} \Delta w \operatorname{sen} \beta\right) \\
V_{n}= & \left(-D \frac{\partial}{\partial r} \Delta w+\frac{1}{r} \frac{\partial m_{n s}}{\partial \theta}\right) \cos \beta-\left(D \frac{1}{r} \frac{\partial}{\partial \theta} \Delta w+\frac{\partial m_{n s}}{\partial r}\right) \operatorname{sen} \beta \\
& +\left(\frac{1}{R}-\frac{\cos \beta}{r}\right) \frac{\partial m_{n s}}{\partial \beta}
\end{aligned}
$$

Ficando a equação diferencial da seguinte forma:

$$
\left(\frac{\partial^{2}}{\partial r^{2}}+\frac{1}{r} \frac{\partial}{\partial r}+\frac{1}{r^{2}} \frac{\partial^{2}}{\partial \theta^{2}}\right)\left(\frac{\partial^{2} w}{\partial r^{2}}+\frac{1}{r} \frac{\partial w}{\partial r}+\frac{1}{r^{2}} \frac{\partial^{2} w}{\partial \theta^{2}}\right)=\frac{g}{D}
$$




\section{3 - Solução fundamental da placas}

Solução fundamental de um problema é a resposta obtida em um ponto $\boldsymbol{p}$ (denominado ponto de campo ou "field point'), qualquer, de um domínio, em geral infinito, consequente de uma carga unitária aplicada num ponto $\boldsymbol{q}$ (denominado ponto de carregamento ou "load point"), também pertencente ao mesmo domínio.

No caso de placas a resposta a ser obtida é o deslocamento transversal $w^{*}$ em $p$, de coordenadas $x(p)$ e $y(p)$, devido à aplicação da carga unitária em $q$, de coordenadas $x(q)$ e $y(q)$. Desta forma, a solução fundamental de placas é obtida pela resolução da equação (II.22), onde o carregamento é a função Delta de Dirac $(\delta(q, p))$, a qual tem as seguintes propriedades:

$$
\begin{array}{ll}
\delta(q, p)=0 & \text { se } \quad p \neq q \\
\delta(q, p)=\infty & \text { se } p=q \\
\int_{\Omega} \delta(q, p) d \Omega=1 &
\end{array}
$$

onde a função $\psi(p)$ é uma função qualquer do domínio $\Omega$.

Assim sendo, pode-se afirmar que:

$$
\int_{\Omega} \psi(p) \delta(q, p) d \Omega=\psi(q)
$$

o que significa dizer que a integral da função Delta de Dirac no domínio é igual à carga unitária. 
Aplicando-se esta função no lugar da carga na equação diferencial da placa tem-se:

$$
\Delta w^{*}=\frac{\delta(q, p)}{D}
$$

Agora pode-se obter a solução fundamental de uma placa, resolvendo a equaçāo anterior a todos os pontos do domínio, desconsiderando-se uma pequena região em torno do "load point" $\boldsymbol{q}$. Assim, a equação é sempre igual a zero. Escrevendo-se a equação diferencial (1l.22) em coordenadas polares, com origem em $\boldsymbol{q}$, e levando-se em conta a simetria, o que a deixa independente de $\theta$, tém-se:

$$
\left[\frac{d^{2}}{d r^{2}}+\frac{1}{r} \frac{d}{d r}\left[\frac{d^{2} w^{*}}{d r^{2}}+\frac{1}{r} \frac{d w^{*}}{d r}\right]=0\right.
$$

A solução desta equação é conhecida e é da forma:

$$
w^{*}=C 1 \cdot \ln r+C 2 \cdot r^{2} \ln r+C 3 \cdot r^{2}+C 4
$$

Duas das condições de contorno, necessárias para resolver II.27, são obtidas analisando-se o domínio finito da placa. Pela simetria considerada em II.26 pode-se afirmar que:

$$
\frac{d w^{*}}{d r}=0 \quad \text { para } \mathrm{r}=0
$$


Em seguida podemos afirmar também que, admitindo-se um círculo de raio $r$ e com o centro em $\boldsymbol{q}$, existirá uma cortante equivalente $V_{n}$, aplicada em todo o contorno do círculo como indicado na figura abaixo,
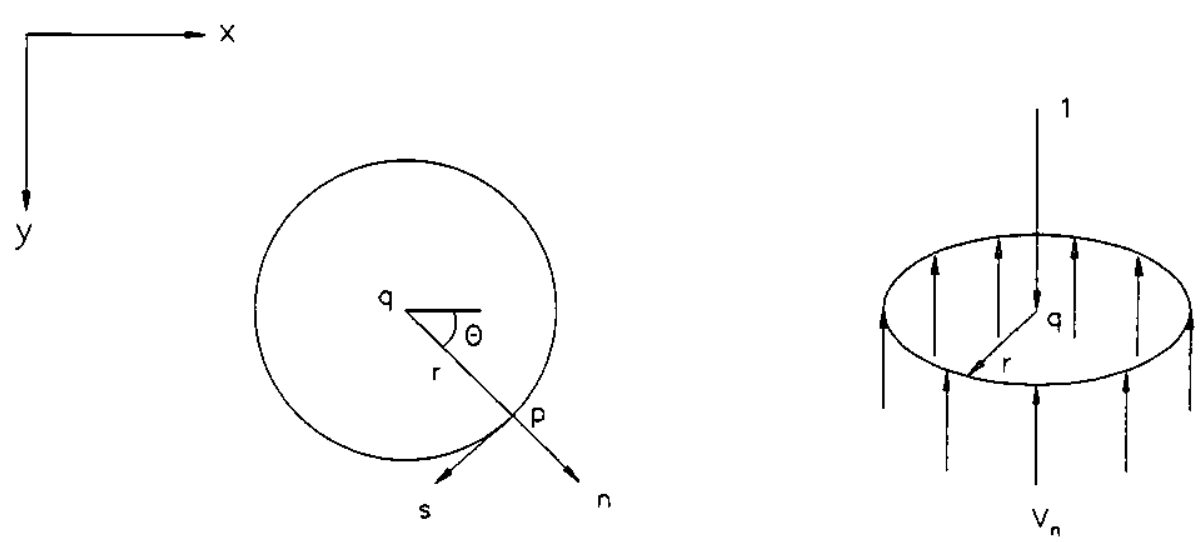

FIGURA 06

cujo equilíbrio é expresso por:

$$
V_{n}=-\frac{1}{2 \pi r}
$$

A expressão da cortante equivalente, escrita em função do deslocamento $w_{p}^{*}$, fica apenas em função de $r$ e com $\circ \beta=0$. Desta forma:

$$
V_{n}=-D \frac{d}{d r} \Delta w^{*}=-\frac{1}{2 \pi r}
$$


Aplicando-se as condições de contorno (II.28) e (II.30) na expressão (II.27) obtém-se as constantes C1 e C2 .

$$
C 1=0 \quad \text { e } \quad C 2=\frac{1}{8 \pi D}
$$

Substituindo-se as constantes acima na expressão (II.27), a equação do deslocamento passa a ser da seguinte forma:

$$
w^{*}=\frac{1}{8 \pi D} r^{2} \ln r+C 3 . r^{2}+C 4
$$

As constantes C3 e C4 podem ser obtidas aplicando-se as condiçōes de contorno do problema da placa a ser analisada. No caso da placa de raio infinito que está sendo aqui analisada, estas condições podem ser quaisquer.

Em DANSON [1979], o autor adota

$$
C 3=-\frac{1}{16 \pi D} \quad \text { e } \quad C 4=0
$$

o que resulta na seguinte equação do deslocamento fundamental $w^{*}$

$$
w^{*}=\frac{1}{8 \pi D} r^{2}\left(\ln r-\frac{1}{2}\right)
$$

Esta é a solução fundamental elastostática de placas, que será utilizada neste trabalho. A partir dela teremos as expressōes de deslocamentos e esforços fundamentais do ponto $\boldsymbol{p}$. Isto se consegue substituindo-se a 
equação acima (II.34) nas expressōes (II.17), (II.18), (II.19) e (II.21). Assim, as expressōes ficam escritas da seguinte forma:

$$
\begin{aligned}
& \frac{\partial w^{*}}{\partial n}=\frac{r}{4 \pi D} \ln r \cos \beta \\
& m_{n}^{*}=-\frac{1}{4 \pi}\left[(1+v) \ln r+(1-v) \cos ^{2} \beta+v\right] \\
& m_{n s}^{*}=\frac{1-v}{8 \pi} \operatorname{sen} 2 \beta \\
& V_{n}^{*}=\frac{\cos \beta}{4 \pi r}\left[2(1-v) \operatorname{sen}^{2} \beta-3+v\right]+\frac{1-v}{4 \pi R} \cos 2 \beta
\end{aligned}
$$

onde:

$$
r=\sqrt{(x(p)-x(q))^{2}+(y(p)-y(q))^{2}}
$$

\section{4 - Equações integrais de placas}

O Método dos Elementos de Contorno se caracteriza pela representação do comportamento de variáveis do domínio através de valores do contorno da placa. Nesse sentido, torna-se necessário obter uma equação integral que relacione o deslocamento(a partir do qual os esforços são escritos) 
de um ponto qualquer pertencente ao domínio $(w(p))$ com os deslocamentos e esforços do contorno.

Esta equação pode ser obtida analisando-se uma placa qualquer, cujo contorno está indicado por $\Gamma$ e o domínio por $\Omega$, que se encontra contida em uma placa de domínio e contorno infinitos, indicados, respectivamente, por $\Gamma_{\infty}$ e por $\Omega_{\infty}$, como pode-se observar na figura 07 abaixo.

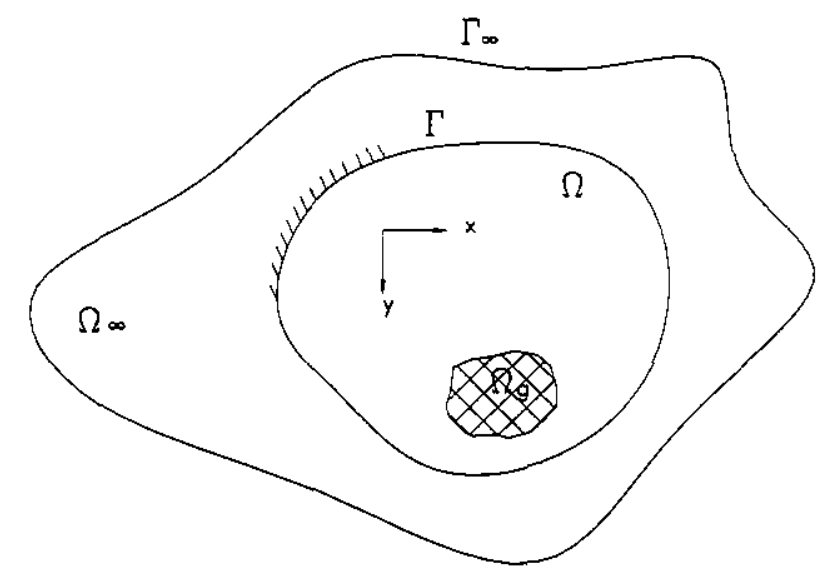

FIGURA 07

Agora considere-se que a placa acima está submetida a dois carregamentos distintos e não simultâneos $\mathbf{g}$ e $\mathbf{g}^{*}$. O primeiro atua no domínio que está indicado na figura acima por $\Omega_{\mathrm{g}}$, enquanto que o segundo é o carregamento correspondente à soluçāo fundamental. Em se considerando estes dois estados de carregamento para a placa, podemos associar à mesma deslocamentos, tensões e deformações distintas. Estes seriam indicados por: $w$ , $\sigma_{i j}$ e $\varepsilon_{i j}$, para o primeiro carregamento, e $w^{*}, \sigma_{i j}^{*}$ e $\varepsilon_{i j}^{*}$, para o carregamento fundamental. Assim sendo, a seguinte igualdade pode ser escrita, de acordo com o teorema de Betti: 


$$
\int_{V} \sigma_{i j}^{*} \varepsilon_{i j} d V=\int_{V} \sigma_{i j} \varepsilon_{i j}^{*} d V \quad 1, \mathrm{j}=1,2,3, \ldots
$$

A partir da expressão acima pretende-se chegar à equação integral desejada. Para tanto, deve-se encontrar uma forma de transformar as integrais de volume, da expressão referida, em integrais de contorno. Para facilitar, primeiramente isso será feito em apenas um dos termos da igualdade.

Tomando-se o termo da direita, e estendendo-se o somatório, podese escrevê-lo como:

$$
\int_{V} \sigma_{i j} \varepsilon_{i j}^{*} d V=\int_{V}\left[\sigma_{x x} \varepsilon_{x x}^{*}+\sigma_{y y} \varepsilon_{y y}^{*}+\sigma_{z z} \varepsilon_{z z}^{*}+\tau_{x y} \gamma_{x y}^{*}+\tau_{x z} \gamma_{x z}^{*}+\tau_{y z} \gamma_{y z}^{*}\right] d V
$$

Esta expressão deve ser agora reescrita levando-se em conta, conforme adotado como hipótese simplificadora, que as tensões relativas à direção normal z são desprezadas.

$$
\int_{V} \sigma_{i j} \varepsilon_{i j}^{*} d V=\int_{V}\left[\sigma_{x} \varepsilon_{x}^{*}+\sigma_{y} \varepsilon_{y}^{*}+\tau_{x y} \gamma_{x y}^{*}\right] d V
$$

Substituindo-se as expressões das tensões e das deformações que se conhece da teoria básica de placas - II.1 e II.2 - na expressão II.42, conseguese deixar a integral apenas em função das derivadas do deslocamento $\boldsymbol{w}$, em relação a $x$ e $y$.

$$
\begin{gathered}
\int_{V} \sigma_{i j} \varepsilon_{i j}^{*} d V=\int_{V}\left[\frac{E}{1-v^{2}}\left(\frac{\partial^{2} w}{\partial x^{2}}+v \frac{\partial^{2} w}{\partial y^{2}}\right) \frac{\partial^{2} w^{*}}{\partial x^{2}} z^{2}+\frac{E}{1-v^{2}}\left(\frac{\partial^{2} w}{\partial y^{2}}+v \frac{\partial^{2} w}{\partial x^{2}}\right) \frac{\partial^{2} w^{*}}{\partial y^{2}} z^{2}\right. \\
\left.+4 G \frac{\partial^{2} w}{\partial x \partial y} \frac{\partial^{2} w^{*}}{\partial x \partial y} z^{2}\right] d V
\end{gathered}
$$


Integrando-se a expressão ao longo da espessura, sendo que esta varia de $-h / 2$ a $+h / 2$, consegue-se transformar a integral de volume acima (II.43) numa integral de domínio bidimensional.

$$
\begin{gathered}
\int_{V} \sigma_{i j} \varepsilon_{i j}^{*} d V=\int_{\Omega}\left[D\left(\frac{\partial^{2} w}{\partial x^{2}}+v \frac{\partial^{2} w}{\partial y^{2}}\right) \frac{\partial^{2} w^{*}}{\partial x^{2}}+D\left(\frac{\partial^{2} w}{\partial y^{2}}+v \frac{\partial^{2} w}{\partial x^{2}}\right) \frac{\partial^{2} w^{*}}{\partial y^{2}}\right. \\
\left.+2 D(1-v) \frac{{ }^{2} w}{\partial x \partial y} \frac{\partial^{2} w^{*}}{\partial x \partial y} z^{2}\right] d \Omega
\end{gathered}
$$

Lembrando-se agora das expressōes (II.4), (II.5) e (II.6), esta integral de domínio pode ser reescrita em função dos momentos e derivadas segundas com relação a $x \in y$.

$$
\int_{V} \sigma_{i j} \varepsilon_{i j}^{*} d V=\int_{\Omega}\left[-m_{x} \frac{\partial^{2} w^{*}}{\partial x^{2}}-m_{y} \frac{\partial^{2} w^{*}}{\partial y^{2}}-2 m_{x y} \frac{\partial^{2} w^{*}}{\partial x \partial y}\right] d \Omega
$$

A redução da integral de volume foi o primeiro passo para se obter a integral de contorno que será utilizado na formulação do MEC. Num segundo passo devem ser levadas em consideração as relaçōes provenientes do teorema geral de Gauss, que são as seguintes:

$$
\begin{aligned}
& \int_{\Omega} \frac{\partial}{\partial x} f(x, y) d x d y=\int_{\Gamma} f(x, y) \eta_{x} d \Gamma \\
& \int_{\Omega} \frac{\partial}{\partial y} f(x, y) d x d y=\int_{\Gamma} f(x, y) h_{y} d \Gamma
\end{aligned}
$$


onde $\eta_{x}$ e $\eta_{y}$ são os cossenos diretores do versor normal ao contorno nas direçōes $x$ e $y$, respectivamente.

De posse destas relações, integra-se por partes o primeiro termo da expressão (II.45), e obtém-se:

$$
-\int_{\Omega} m_{x} \frac{\partial^{2} w^{*}}{\partial x^{2}} d \Omega=-\int_{\Gamma} m_{x} \frac{\partial w^{*}}{\partial x} \cos \alpha d \Gamma+\int_{\Omega} \partial^{w^{*}} \frac{\partial m_{x}}{\partial x} d \Omega
$$

Integrando-se por partes a segunda parcela da expressão anterior (II.47) pode-se escrever boa parte do primeiro termo da expressão (II.45) sob a forma de integrais de contorno.

$$
-\int_{\Omega} m_{x} \frac{\partial^{2} w^{*}}{\partial x^{2}} d \Omega=\int_{\Gamma}\left[-m_{x} \frac{\partial w^{*}}{\partial x} \cos \alpha+\frac{\partial m_{x}}{\partial x} w^{*} \cos \alpha\right] \Gamma-\int_{\Omega} \frac{\partial^{2} m_{x}}{\partial x^{2}} w^{*} d
$$

Seguindo-se o mesmo roteiro, adotado para se chegar à expressão anterior, pode-se escrever o segundo e o terceiro termo de (II.45).

$-\int_{\Omega} m_{y} \frac{\partial^{2} w^{*}}{\partial y^{2}} d \Omega=\int_{\Gamma}-\left[m_{y} \frac{\partial w^{*}}{\partial y} \operatorname{sen} \alpha+\frac{\partial m_{y}}{\partial y} w^{*} \operatorname{sen} \alpha\right] d \Gamma-\int_{\Omega} \frac{\partial^{2} m_{y}}{\partial y^{2}} w^{*} d \Omega$

$$
\begin{gathered}
-2 \int_{\Omega} m_{x y} \frac{\partial^{2} w^{*}}{\partial x \partial y} d \Omega=\int_{\Gamma}-m_{x y} \frac{\partial w^{*}}{\partial y} \cos \alpha-m_{x y} \frac{\partial w^{*}}{\partial x} \operatorname{sen} \alpha+\frac{\partial m_{x y}}{\partial x} w^{*} \operatorname{sen} \alpha \\
\left.+\frac{\partial m_{x y}}{\partial y} w^{*} \cos \alpha\right] d \Gamma-\int_{\Omega} 2 \frac{\partial m_{x y}}{\partial y} w^{*} d
\end{gathered}
$$


Estes três termos voltam a ser agrupados para reescrever II.45, que agora já está em parte representado por integrais de contorno. A expressão fica escrita da seguinte forma:

$$
\begin{aligned}
\int_{V} \sigma_{i j} \varepsilon_{i j}^{*} d V & =-\int_{\Gamma}\left[m_{x} \frac{\partial w^{*}}{\partial x} \cos \alpha+m_{y} \frac{\partial w^{*}}{\partial y} \operatorname{sen} \alpha+m_{x y} \frac{\partial w^{*}}{\partial y} \cos \alpha+m_{x y} \frac{\partial w^{*}}{\partial x} \operatorname{sen} \alpha\right] \Gamma \\
& +\int_{\Gamma}\left[\left(\frac{\partial m_{x}}{\partial x}+\frac{\partial m_{x y}}{\partial y}\right) \cos \alpha+\left(\frac{\partial m_{y}}{\partial y}+\frac{\partial m_{x y}}{\partial x}\right) \operatorname{sen} \alpha\right] w^{*} d \Gamma-\int\left[\frac{\partial^{2} m_{x}}{\partial x^{2}}\right. \\
& \left.+\frac{\partial^{2} m_{y}}{\partial y^{2}}+2 \frac{\partial^{2} m_{x y}}{\partial x \partial y}\right] w^{*} d \Omega
\end{aligned}
$$

Essa expressão pode ainda ser simplificada caso se utilize a relação (II.3). Além disso ser feito, deve-se escrevê-la com referência aos semieixos $n$ e s. Para tanto deve-se relacionar as derivadas de $w$ em relação a $x e$ $y$ com as mesmas só que em relação ao novo sistema de referência. Isto é simples de ser feito, bastando observar as seguintes igualdades:

$$
\begin{aligned}
& \frac{\partial w^{*}}{\partial x}=\frac{\partial w^{*}}{\partial n} \cos \alpha-\frac{\partial w^{*}}{\partial s} \operatorname{sen} \alpha \\
& \frac{\partial w^{*}}{\partial y}=\frac{\partial w^{*}}{\partial n} \operatorname{sen} \alpha+\frac{\partial w^{*}}{\partial s} \cos \alpha
\end{aligned}
$$

Uma vez feita a substituição das relações escritas em (II.52) na expressão 11.51 , pode-se simplificá-la lembrando-se das expressōes 11.10 a II.12. 


$$
\int_{V} \sigma_{i j} \varepsilon_{i j}^{*} d V=-\int_{\Gamma}\left[m_{n} \frac{\partial w^{*}}{\partial n}+m_{n s} \frac{\partial w^{*}}{\partial s}-q_{n} \cdot w^{*}\right] d \Gamma+\int_{\Omega} g w^{*} d \Omega
$$

Integrando-se por partes o termo que contém $m_{n s}$, tém-se que

$$
\int_{\Gamma} m_{n s} \frac{\partial w^{*}}{\partial s} d \Gamma=\left.m_{n s} w^{*}\right|_{\Gamma_{1}} ^{\Gamma_{2}}-\int_{\Gamma} \frac{\partial m_{n s}}{\partial s} w^{*} d \Gamma
$$

onde $\Gamma_{1}$ e $\Gamma_{2}$ são os limites de integração ao longo de s. Se a integração é feita numa placa de contorno fechado e que não contenha cantos, a primeira parcela se anula, já que não há descontinuidade de $\boldsymbol{n}$ e $\boldsymbol{s}$, sendo, portanto, o $\boldsymbol{m}_{n s}$ contínuo. No caso de haver canto na placa, o resultado da aplicação dos diferentes limites de integraçāo será a reação $R_{c}$, referente a esse canto. Se a placa tem vários cantos, esta parcela é o somatório de todas as reações $\boldsymbol{R}_{c}$. Para melhor ilustrar o acima descrito, deve-se acompanhar a demonstraçāo feita a partir da figura 08 .

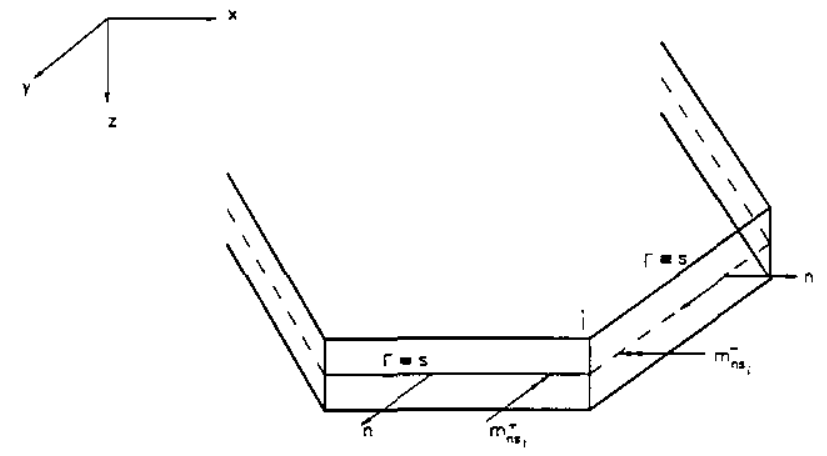

FIGURA 08 
Escrevendo-se o somatório para todos os cantos da placa onde se aplicam os limites de integração, tém-se:

$$
\left.\sum_{l=1}^{N l} m_{n s_{i}} w_{l}^{*}\right|_{\Gamma_{t l}} ^{\Gamma_{i 2}}=-\sum_{i=1}^{N c_{c}}\left(m_{n s_{i}}^{+}-m_{n s_{i}}^{-}\right) w_{c_{i}}^{*}=-\sum_{i=1}^{N c_{c}} R_{c_{i}} w_{c_{i}}^{*}
$$

sendo $N_{l}$ e $N_{c}$ os números de lados e cantos da placa, respectivamente, $w_{c i}{ }^{*} \circ$ deslocamento fundamental, relativo ao canto $i$, e $\Gamma_{11}$ e $\Gamma_{12}$ os limites de integração referentes a cada lado da placa.

Substituindo-se, primeiramente, (II.55) em (II.54), e o resultado disso em (II.53), e levando-se em conta a relação (II.13), obtém-se a expressão final de 11.41 .

$$
\left.\int_{V} \sigma_{i j} \varepsilon_{i j}^{*} d V=\int_{\Gamma^{2}} V_{n} w^{*}-m_{n} \frac{\partial w^{*}}{\partial n}\right] d \Gamma+\sum_{i=1}^{N c} R_{c_{i}} w_{c_{i}}^{*}+\int_{\Omega_{g}} g w^{*} d \Omega_{g}
$$

Fazendo-se, de forma análoga, todos os passos para o termo da esquerda da igualdade advinda do teorema de Betti pode-se afirmar que:

$$
\int_{V} \sigma_{i j}^{*} \varepsilon_{i j} d V=\int_{\Gamma}\left[V_{n}^{*} w-m_{n}^{*} \frac{\partial w}{\partial n}\right] d \Gamma+\sum_{i=1}^{N_{c_{c}}} R_{c_{i}}^{*} w_{c_{i}}+\int_{\Omega} g^{*} w d \Omega
$$

Assim, a igualdade (II.40) pode ser reescrita deixando de ficar expressa em integrais de volume e passando a ser expressa em integrais de contorno, a menos das integrais de domínio referentes aos carregamentos $\mathrm{g}$ e $g^{*}$. Sabe-se também que os deslocamentos e os esforços referentes ao carregamento fundamental $g^{*}$ são funçōes que dependem do "load point" $q$ e 
do ponto de deslocamento $\boldsymbol{p}$, ao passo que os referentes ao carregamento $\boldsymbol{g}$ são funçōes que dependem apenas de $p$, que é o ponto onde os efeitos da carga são determinados. Assim sendo:

$$
\begin{aligned}
& \int_{\Gamma}\left[V_{n}^{*}(q, p) w(p)-m_{n}^{*}(q, p) \frac{\partial w(p)}{\partial n} d \Gamma_{(p)}+\sum_{i=1}^{N_{c}} R_{c_{i}}^{*}(q, p) w_{c_{i}}(p)\right. \\
& +\int_{\Omega_{s}} g^{*}(q, p) w(p) d \Omega_{g_{(p)}}=\int_{\Gamma}\left[V_{n}(p) w^{*}(q, p)-m_{n}(p) \frac{\left.\partial w^{*}(q, p)\right]}{\partial n}\right] \Gamma_{(p)} \\
& +\sum_{i=1}^{N_{c}} R_{c_{i}}(p) w_{c_{i}}^{*}(q, p)+\int_{\Omega_{s}} g(p) w^{*}(q, p) d \Omega_{g_{(p)}}
\end{aligned}
$$

Esta é a equação integral de placas que será utilizada na formulação do Método dos Elementos de Contorno, como se verá no ítem a seguir. O tratamento das duas integrais de domínio remanescentes, relativas aos dois tipos de carregamento, também será visto no próximo ítem.

\section{5 - Método dos elementos de contorno}

\subsection{1 - Solução das equações integrais}

O Método dos Elementos de Contorno consiste em transformar a equação integral $11.58 \mathrm{em}$ uma equação algébrica, resolvendo-a 
numericamente. A opção em resolvê-la numericamente se deve ao fato de ser impossivel a sua resolução analítica.

Para se fazer essa transformação, deve-se discretizar o contorno da placa em "elementos". É sobre esses elementos que os deslocamentos e esforços da equação integral serão aproximados, utilizando-se funções interpoladoras. Essas funções aproximam os valores de $w, \partial w / \partial n, V_{n} \in m_{n}, a$ partir de valores nodais e das funçōes aproximadoras adotadas.

Estas funçōes são definidas pelo tipo de elemento que está sendo utilizado. No caso de elementos com geometria linear, os tipos são: elemento constante (com aproximação constante), linear (aproximação linear, necessitando de 2 pontos nodais para definir as funções), quadrático (aproximação quadrática, necessitando de 3 pontos nodais) entre outros.

A discretização das equações algébricas conduz a um sistema de equaçōes lineares onde as incógnitas nodais são os deslocamentos $e$ os esforços. No caso de placas, duas condições de contorno são conhecidas para cada nó, restando duas incógnitas nodais. Desta forma, torna-se necessário que se escrevam duas equaçōes para cada nó, para que se tenha um número de incógnitas igual ao número de equações. Comumente, PAIVA (1990), OLIVEIRA NETO (1991), escreve-se a equação do deslocamento $w$ e uma equação referente à derivada de $\boldsymbol{w}$ em relação à coordenada $\boldsymbol{m}$, de um sistema de eixos cartesianos $\boldsymbol{m}$ e $\boldsymbol{u}$, com origem no ponto $\boldsymbol{p}$ (ponto de deslocamento). No presente trabalho optou-se por escrever duas equaçōes para o deslocamento $w$, escritas a partir de dois pontos situados fora do domínio [TEJERINA CALDERÓN (1991)]. Esses pontos estão situados a uma distância que corresponde à metade do comprimento do elemento, para o primeiro ponto, seguindo uma direçāo normal ao elemento, e igual ao comprimento do elemento, para o segundo ponto. Essas distâncias podem variar sem provocar alteraçōes na precisão dos resultados. Essa alternativa adotada apresenta a 
vantagem de não se ter os problemas de singularidade, que costumam acorrer nos casos onde os pontos fonte se encontram no contorno. Uma outra opção para resolver o problema das integrais singulares é utilizar-se da integração numérica via algoritmo de Kutt [KUTT (1975)], porém a simplicidade de se colocar os pontos fora do domínio justificou a adoção dessa forma de resolver as integrais singulares. Os resultados obtidos, usando esta alternativa, se mostram bastante satisfatórios.

Assim têm-se dois tipos de equações: uma escrita para os pontos situados no domínio, pontos internos, e outra escrita para os pontos situados fora do domínio, pontos externos, associados aos nós. Estas equações diferem entre si pela forma com que a integral de domínio, referente ao carregamento fundamental $\boldsymbol{g}^{*}$, é resolvida.

Sabe-se que a carga fundamental adotada é a função Delta de Dirac. Observando-se as propriedades desta função (II.23), pode-se reescrever as integrais de domínio, referentes a $g^{*}$.

Para o caso de pontos internos:

$$
\int_{\Omega} g^{*}(q, p) w(p) d \Omega_{(p)}=w(q)
$$

ficando a equação integral da seguinte forma:

$$
w(q)+\int_{\Gamma}\left[V_{n}^{*}(q, p) w(p)-m_{n}^{*}(q, p) \frac{\partial w(p)}{\partial n}\right] d \Gamma_{(p)}+\sum_{i=1}^{N_{c}} R_{c_{i}}^{*}(q, p) w_{c_{i}}(p)
$$


31

$$
\begin{aligned}
& =\int_{\Gamma}\left[V_{n}(p) w^{*}(q, p)-m_{n}(p) \frac{\partial w^{*}(q, p)}{\partial n}\right] d \Gamma_{(p)}+\sum_{i=1}^{N_{c}} R_{c_{i}}(p) w_{c_{i}}^{*}(q, p) \\
& +\int_{\Omega_{s}} g(p) w^{*}(q, p) d \Omega_{g_{(p)}}
\end{aligned}
$$

Para os pontos externos, a integral acima passa a ser escrita para um ponto fonte a (situado fora do domínio), e isto faz com que a integral de domínio se anule,

$$
\int_{\Omega} g^{*}(q, p) w(p) d \Omega_{(p)}=0
$$

ficando a equação integral reduzida a:

$$
\begin{aligned}
& \int_{\Gamma}\left[V_{n}^{*}(a, p) w(p)-m_{n}^{*}(a, p) \frac{\partial w(p)}{\partial n}\right] d \Gamma_{(p)}+\sum_{i=1}^{N_{c}} R_{c_{i}}^{*}(a, p) w_{c_{i}}(p) \\
& =\int_{\Gamma}\left[V_{n}(p) w^{*}(a, p)-m_{n}(p) \frac{\partial w^{*}(a, p)}{\partial n}\right] d \Gamma_{(p)}+\sum_{i=1}^{N c_{c}} R_{c_{i}}(p) w_{c_{t}}^{*}(a, p) \\
& +\int_{\Omega_{\varepsilon}} g(p) w^{*}(a, p) d \Omega_{g_{(p)}}
\end{aligned}
$$




\subsection{2 - Definição do elemento}

O tipo de elemento adotado é o elemento quadrático contínuo. Consequentemente, conforme já definido anteriormente, as funçōes aproximadoras são quadráticas, tornando-se necessário a definição de 3 nós por elemento. Nas figuras que se seguem pode-se observar um exemplo de discretização do contorno de uma placa quadrada em elementos quadráticos (fig. 09.a) e um exemplo da aproximaçāo do deslocamento $w$ ao longo de um elemento (fig. 09.b). Nestas figuras os nós duplos, além do próprio nó de canto, têm as mesmas coordenadas. Embora eles sugiram a existência de elementos descontínuos, esta forma de representá-los foi escolhida para ressaltar a existência de diferentes normais para cada nó duplo.

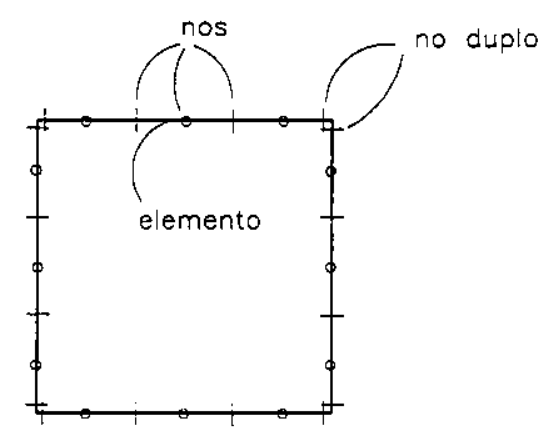

(a)

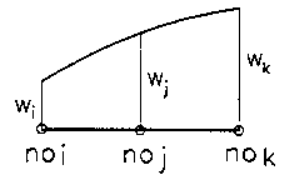

(b)

FIGURA 09

Com a colocação desses nós duplos nos cantos permite-se que cada um deles assuma valores nodais diferentes, apesar de terem as mesmas coordenadas. Na figura 10, que se segue, estão representados os pontos de carregamento, ou pontos fonte, dos nós representados na figura 09 anterior. 
Nota-se que a colocação desses ponto segue a orientação descrita no item anterior.

Como será escrita uma equação, separadamente, para cada canto, os pontos fonte ficam localizados a uma distância correspondente à metade do elemento, na direção da bissetriz formada pelos dois elementos que o geram.

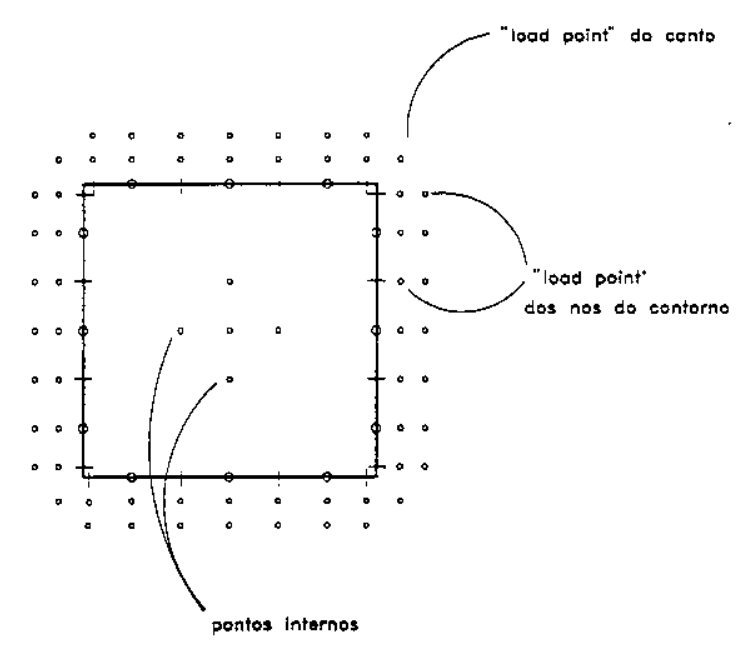

FIGURA 10

Assim, escrevem-se duas equações para cada nó do contorno, além de uma para cada canto. Com isto são cinco equações escritas para cada canto, sendo duas referentes a cada um dos nós duplos e uma do canto. No caso dos pontos de domínio já se sabe que os pontos de carregamento coincidem com os mesmos.

A partir da figura (09.b) definem-se as funções interpoladoras do elemento quadrático. Com estas funções é possivel obter quaisquer valores (coordenadas, deslocamentos, etc.) ao longo do elemento, em função dos valores assumidos pelos nós do elemento. Ao longo deste trabalho estas 
funçōes serão sempre reconhecidas pelo símbolo $\phi$. No caso do elemento quadrático continuo, estas funçōes são:

$$
\begin{aligned}
& \phi_{1}=\frac{1}{2} \xi(\xi-1) \\
& \phi_{2}=(1-\xi)(1+\xi) \\
& \phi_{3}=\frac{1}{2} \xi(\xi+1)
\end{aligned}
$$

As funções $\phi$ têm a propriedade de serem iguais a 1 nos respectivos nós e zero nos demais. De posse destas, conforme já foi dito no parágrafo anterior, pode-se escrever que

$$
w=\left[\phi_{i}\right]\left\{w_{i}\right\}_{\Gamma_{m}}
$$

sendo que $i$ indica o número do nó, e $\boldsymbol{m}$ indica o número do elemento.

Da mesma forma os outros valores que estão presentes na equação integral das placas podem ser aproximados. Estes valores, reescritos em função dos valores nodais, serão substituídos na equação integral transformando-a numa equação algébrica que será escrita de forma matricial. Os valores restantes são:

$$
\frac{\partial w}{\partial \eta}=\left[\phi_{i}\right]\left\{\left(\frac{\partial w}{\partial n}\right)_{i}\right\}_{\Gamma_{m}}
$$




$$
\begin{aligned}
& m_{n}=\left[\phi_{i}\right]\left\{m_{n_{i}}\right\}_{\Gamma_{m}} \\
& V_{n}=\left[\phi_{i}\right]\left\{V_{n_{i}}\right\}_{\Gamma_{m}}
\end{aligned}
$$

\subsection{3 - Equações matriciais para o elemento quadrático}

Inicialmente convém lembrar que a placa está dividida em $N_{0}$ elementos de contorno, conforme ilustrado na figura (09.a), e que os valores incógnitos encontrados nas equações integrais (II.60) e (II.62) podem ser escritos da forma mostrada em (II.64), (II.65), (II.66) e (II.67). Assim sendo uma equação algébrica, para um ponto qualquer $\boldsymbol{q}$, pode ser escrita a partir da equações integrais, bastando que estas passem a estar escritas em função dos valores nodais. Esta transformação das integrais de contorno para integrais em função dos valores nodais, nada mais é do que o somatório destas, escrito para todos os elementos da placa.

$$
\begin{aligned}
& \sum_{m=1}^{N c_{\Gamma_{m}}}\left\{\left\{[\phi(p)]\left\{w_{i}\right\}_{\Gamma_{*}} V_{n}^{*}(q, p)-[\phi(p)]\left\{\left(\frac{\partial w}{\partial n}\right)_{i}\right\}_{\Gamma_{\sigma_{*}}} m_{n}^{*}(q, p)\right\} d \Gamma_{(p)}+\sum_{i=1}^{N_{c_{i}}} R_{c_{i}}^{*}(q, p) w_{c_{i}}(p)\right.
\end{aligned}
$$

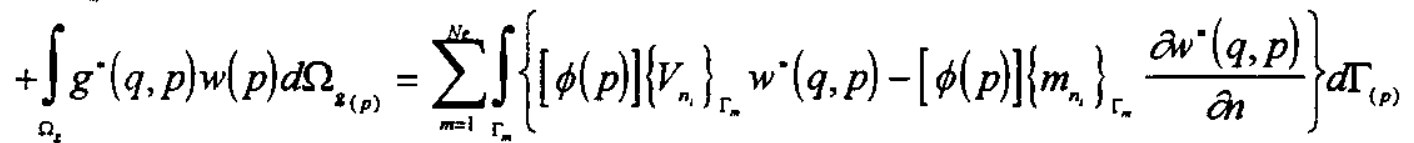

$$
\begin{aligned}
& +\sum_{i=1}^{N c} w_{c_{i}}^{*}(q, p) R_{c_{i}}(p)+\int_{\Omega_{s}} g(p) w^{*}(q, p) d \Omega_{g_{(p)}}
\end{aligned}
$$

onde $N_{\mathrm{e}}$ significa o número total de elementos. 
36

A equação anterior deve agora ser definida para os dois tipos de pontos a partir dos quais ela será escrita, extendendo a sua forma para o caso do tipo de elemento adotado.

No caso dos pontos externos:

$$
\begin{aligned}
& \sum_{m=1}^{N_{c}}\left\{w_{i} \int_{\Gamma_{m}} \phi_{1}(p) V_{n}^{*}(q, p) d \Gamma_{m(p)}+w_{j} \int_{\Gamma_{m}} \phi_{2}(p) V_{n}^{*}(q, p) d \Gamma_{m(p)}+w_{k} \int_{\Gamma_{m}} \phi_{3}(p) V_{n}^{*}(q, p) d \Gamma_{m(p)}\right. \\
& \left.-\frac{\partial w_{i}}{\partial n} \int_{\Gamma} \phi_{1}(p) m_{n}^{*}(q, p) d \Gamma_{m(p)}-\frac{\partial w_{j}}{\partial n} \int_{\Gamma_{m}} \phi_{2}(p) m_{n}^{*}(q, p) d \Gamma_{m(p)}-\frac{\partial w_{k}}{\partial n} \int_{\Gamma_{m}} \phi_{3}(p) m_{n}^{*}(q, p) d \Gamma_{m(p)}\right\} \\
& +\sum_{i=1}^{R_{c}} R_{c_{i}}^{*}(q, p) w_{c_{i}}(p)=\sum_{m=1}^{N_{e}}\left\{V_{n_{i}} \int_{\Gamma_{m}} \phi_{1}(p) w^{*}(q, p) d \Gamma_{m(p)}+V_{n_{j}} \int_{\Gamma_{m}} \phi_{i}(p) w^{*}(q, p) d \Gamma_{m(p)}\right. \\
& +V_{n_{k}} \int_{\Gamma_{m}} \phi_{3}(p) w^{*}(q, p) d \Gamma_{m(p)}-m_{r_{i}} \int_{\Gamma_{m}} \phi_{1}(p) \frac{\partial w^{*}(q, p)}{\partial n} d \Gamma_{m(p)}-m_{n_{j}} \int_{\Gamma_{m}} \phi_{2}(p) \frac{\partial w^{*}(q, p)}{\partial n} d \Gamma_{m(p)} \\
& \left.-m_{n_{k}} \int_{\Gamma_{m}} \phi_{3}(p) \frac{\partial w^{*}(q, p)}{\partial n} d \Gamma_{m(p)}\right\}+\sum_{i=1}^{N c_{c}} w^{*}(q, p) R_{c_{i}}(p)+\int_{\Omega_{i}} g(p) w^{*}(q, p) d \Omega_{g_{(p)}}
\end{aligned}
$$

No caso dos pontos internos:

$$
\begin{aligned}
& w(q)+\sum_{m=1}^{N_{e}}\left\{w_{i} \int_{\Gamma_{m}} \phi_{1}(p) V_{n}^{*}(q, p) d \Gamma_{m(p)}+w_{j} \int_{\Gamma_{m}} \phi_{2}(p) V_{n}^{*}(q, p) d \Gamma_{m(p)}+w_{k} \int_{\Gamma_{m}} \phi_{3}(p) V_{n}^{*}(q, p) d \Gamma_{m(p)}\right. \\
& \left.-\frac{\partial w_{i}}{\partial n} \int_{\Gamma} \phi_{1}(p) m_{n}^{*}(q, p) d \Gamma_{m(p)}-\frac{\partial w_{j}}{\partial n} \int_{\Gamma_{m}} \phi_{2}(p) m_{n}^{*}(q, p) d \Gamma_{m(p)}-\frac{\partial w_{k}}{\partial n} \int_{\Gamma_{m}} \phi_{3}(p) m_{n}^{*}(q, p) d \Gamma_{m(p)}\right\}
\end{aligned}
$$




$$
\begin{aligned}
& +\sum_{i=1}^{R c_{c}} R_{c_{i}}^{*}(q, p) w_{c_{i}}(p)=\sum_{m=1}^{N_{e}}\left\{V_{n_{i}} \int_{\Gamma_{m}} \phi_{1}(p) w^{*}(q, p) d \Gamma_{m(p)}+V_{n_{j}} \int_{\Gamma_{m}} \phi_{2}(p) w^{*}(q, p) d \Gamma_{m(p)}\right. \\
& +V_{n_{k}} \int_{\Gamma_{m}} \phi_{3}(p) w^{*}(q, p) d \Gamma_{m(p)}-m_{n_{i}} \int_{\Gamma_{m}} \phi_{1}(p) \frac{\partial w^{*}(q, p)}{\partial n} d \Gamma_{m(p)}-m_{n_{j}} \int_{\Gamma_{m}} \phi_{2}(p) \frac{\partial w^{*}(q, p)}{\partial n} d \Gamma_{m(p)} \\
& \left.-m_{n_{n_{k}}} \int_{\Gamma_{m}} \phi_{3}(p) \frac{\partial w^{*}(q, p)}{\partial n} d \Gamma_{m(p)}\right\}+\sum_{l=1}^{N_{c}} w^{*}(q, p) R_{c_{i}}(p)+\int_{\Omega_{z}} g(p) w^{*}(q, p) d \Omega_{z_{(p)}}
\end{aligned}
$$

O número de equações escritas é igual ao número de pontos de carregamento que se tem no problema em questão. Em outras palavras, será igual a duas vezes o número de nós do contorno mais o número de cantos da placa mais o número de pontos internos. Como existem duas incógnitas para cada nó do contorno, uma para cada canto e uma para cada ponto interno, tém-se um número de equações igual ao número de incógnitas, e o sistema pode ser resolvido. Esse sistema pode ser escrito sob a forma matricial,

$$
\left[\begin{array}{ccc}
\underset{\sim \Gamma}{H} & \underset{\sim}{H} & \underset{\sim}{H}
\end{array}\right]\left[\begin{array}{l}
\underset{\sim}{w} \\
\underset{\sim}{w} \\
\underset{\sim}{w} \\
\underset{\sim}{w}
\end{array}\right\}=\left[\begin{array}{ll}
\underbrace{G}_{\Gamma} & \underset{\sim}{G}
\end{array}\right]\left\{\begin{array}{l}
\underset{\sim}{P} \\
\underset{\sim}{P}
\end{array}\right\}+\{F\}
$$

onde o sinal subscrito $\sim$, sob as letras, indicam que estas são sub-matrizes, no caso de $\boldsymbol{H}$ e de $\boldsymbol{G}$, ou sub-vetores, no caso de $\boldsymbol{w}$ e de $\boldsymbol{P}$. Os índices indicam que são referentes ao contorno, aos cantos ou aos pontos internos. $O$ vetor indicado por $\boldsymbol{F}$ é o vetor resultante do carregamento externo aplicado à placa.

Essas sub-matrizes e sub-vetores são as seguintes: 


$$
\begin{aligned}
& \underset{\sim \mathrm{r}}{w}=\left\{\begin{array}{l}
w(q) \\
\frac{\partial w(q)}{\partial n}
\end{array}\right\} \\
& \underset{\sim \Gamma}{P}=\left\{\begin{array}{l}
V_{n}(q) \\
m_{n}(q)
\end{array}\right\}
\end{aligned}
$$

As dimensōes de cada uma das sub-matrizes e sub-vetores está indicada a seguir.

$$
\begin{aligned}
& \underset{\sim \Gamma}{H}-\text { NVTT } \times \text { NTOT } \\
& \underset{\sim c}{H}-\text { NVTT } \times \text { NCAN } \\
& \underset{\sim}{H}-\text { NVTT } \times \text { NNI }
\end{aligned}
$$

as matrizes $\underset{\sim_{\Gamma}}{G} e \underset{\sim_{C}}{G}$ têm um número de colunas igual às respectivas matrizes $H$, sendo que o número de linhas é igual a NTOT+NCAN.

$\underset{\sim \Gamma}{w} \underset{\sim \Gamma}{P}-$ NTOT, e indicam o vetor de deslocamentos ( w e $\partial w / \partial n$ ) e de esforços $\left(V_{n}\right.$ e $\left.m_{n}\right)$

${\underset{\sim}{c}}_{c} e{\underset{\sim}{c}}_{\text {- }}$ - NCAN, e indicam o vetor de deslocamentos e das reações dos cantos

$\underset{\sim}{w}-\mathrm{NNI}, \mathrm{e}$ indicam o vetor de deslocamentos dos pontos internos 
Finalmente, aplicando-se as condiçōes de contorno obtém-se o resultado do sistema de equações e determinam-se os deslocamentos e esforços na placa.

Convém esclarecer, entretanto, que a inlcusão dos pontos internos na resolução do sistema não é a forma mais adequada, devido ao fato dela não ser muito "econômica" na hora da resolução. A melhor forma seria resolver primeiro o contorno da placa para, em seguida, calcular os valores dos pontos internos, com os resultados obtidos do contorno. A explicação para terse optado pela inclusão dos pontos internos se deve ao fato deles serem necessários na integração do domínio, como será visto no capítulo seguinte,

\subsection{4 - Montagem das matrizes $H$ e $G$}

Para melhor esclarecer como o sistema (Il.71) é formado, a partir das equações (II.69) e (II.70), procede-se pela representação de cada uma das sub-matrizes indicadas no ítem anterior, começando pelas sub-matrizes do contorno.

Estas são formadas pelo resultado das integrais indicadas em (II.69) e (II.70), as quais são resolvidas numericamente, empregando-se a Quadratura de Gauss. Assim sendo, 12 valores são calculados (6 para cada sub-matriz) para cada elemento podendo haver sobreposição de alguns deles, no caso de nós comuns que pertençam a elementos diferentes. Isto é feito a partir de cada um dos pontos de carregamento da placa, gerando um número de linhas igual a NVTT. O número de colunas será igual a duas vezes o número de pontos do contorno. 
A forma de empregar a Quadratura de Gauss para resolver numericamente as integrais está detalhada no apêndice $\mathbf{A}$.

As sub-matrizes referentes aos cantos são geradas calculando-se os valores presentes nos somatórios, a partir de cada um dos pontos de carregamento. Desta forma NVTT linhas de NCAN colunas são geradas. Quanto à sub-matriz referente aos pontos internos esta é uma matriz identidade de dimensões NNI. As colunas restantes, que ficam acima da identidade, são todas formadas de zeros.

Reescrevendo-se o sistema (II.71), de acordo com as explicações acima, tém-se que:

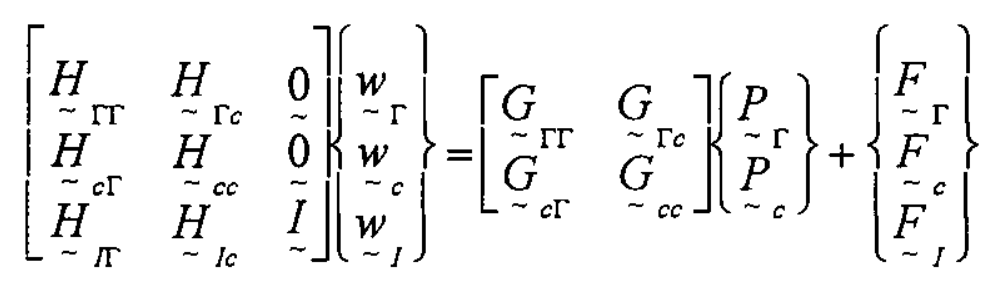

sendo que o primeiro índice indica a região onde está o ponto de carregamento $\boldsymbol{q}$ e o segundo indica a região onde está o ponto de deslocamento $\boldsymbol{p}$.

\subsection{5 - Integral de dominio para montagem do vetor $F$}

Das equações (II.69) e (II.70) resta determinar os valores da integral de domínio

$$
\int_{\Omega_{g}} g(p) w^{*}(q, p) d \Omega_{g(p)}
$$


No caso do carregamento distribuido isto também será feito numericamente através da quadratura de Gauss, e os detalhes desta resolução também estão no apêndice $\mathbf{A}$.

Da mesma forma como foi feito na integração sobre o elemento, na integração do domínio torna-se conveniente subdividí-lo em subdomínios. Com esta subdivisão consegue-se representar melhor o carregamento distribuído

Dividindo-se o domínio em sub-domínios, pode-se escrever o valor do carregamento distribuido, para um ponto de carregamento qualquer $q$, como sendo o somatório da forma:

$$
f d(q)=g(p) \sum_{k=1}^{N S U R} \sum_{j=1}^{N G i} \sum_{i=1}^{N G i} J\left(\xi_{i}\right) J\left(\eta_{j}\right) w_{i} w_{j} w^{*}(q, p)
$$

Para o caso de existirem cargas concentradas, a parcela correspondente a este tipo de carregamento pode ser calculada como sendo o produto da carga pelo valor do deslocamento fundamental, no ponto de aplicação da carga, ou seja:

$$
f d q)=g(p) w^{*}(q, p)
$$

$O$ vetor de cargas $\boldsymbol{F}$ será a soma dos vetores resultantes dos carregamentos distribuído e concentrado, e terá dimensão de NVTT. 


\subsection{6 - Condições de contorno}

No momento de se resolver o sistema de equações é necessário que se imponham as condições de contorno, características do problema. Conforme já foi comentado anteriormente, para cada nó do contorno duas condiçōes são conhecidas. Estas condições de contorno sāo as variáveis de deslocamento $w$ e $\partial w / \partial n$ e de esforços $\boldsymbol{V}_{n}$ e $\boldsymbol{m}_{n}$. No caso dos cantos e dos pontos internos apenas uma variável é conhecida já que estes pontos só têm duas incógnitas (deslocamento w e reação $R$ ).

As condições de contorno para os casos mais comuns de vinculações de placas são:

- Borda simplesmente apoiada

$$
\begin{array}{ll}
\text { we } \mathrm{m}_{\mathrm{n}} & \text { conhecidos } \\
\mathrm{V}_{\mathrm{n}} \mathrm{e} \frac{\partial w}{\partial n} & \text { desconhecidos }
\end{array}
$$

- Borda engastada

$$
\begin{array}{ll}
\text { we } \frac{\partial w}{\partial n} & \text { conhecidos } \\
v_{n} \text { e } m_{n} & \text { desconhecidos }
\end{array}
$$

- Borda livre

$$
\begin{array}{ll}
V_{n} \text { e } m_{n} & \text { conhecidos } \\
w e \frac{\partial w}{\partial n} & \text { desconhecidos }
\end{array}
$$




\subsection{7 - Montagem e solução do sistema final}

Depois de montadas as matrizes e o vetor de carregamento, témse o seguinte sistema de equações:

$$
\underset{\sim}{H} \underset{\sim}{U}=\underset{\sim}{G} \underset{\sim}{P}+\underset{\sim}{F}
$$

Como se sabe, $\boldsymbol{U}$ indica o vetor de deslocamentos ( $\boldsymbol{w}$ e $\partial w / \partial n$ ) enquanto $P$ indica o vetọr dos esforços $\left(V_{n}\right.$ e $\left.m_{n}\right)$. Com as condições de contorno do problema em questão, duas dessas variáveis (deslocamentos ou esforços) são conhecidas para cada nó. Assim sendo procede-se uma troca de posição entre as matrizes $H$ e $G$, da mesma forma que entre os vetores $\boldsymbol{U} \in \boldsymbol{P}$, resultando no seguinte sistema,

$$
\underset{\sim}{A} \underset{\sim}{X}=\underset{\sim}{Q}
$$

onde o vetor $X$ passa a ser o vetor que contém todas as incógnitas do sistema, e o vetor $\boldsymbol{Q}$ seria o resultado da soma do vetor $\boldsymbol{F}$ com o produto da nova matriz $G$ pelo vetor com as condições de contorno do problema. Uma vez resolvido o sistema acima, todas as variáveis passam a ser conhecidas e o problema está resolvido. 


\section{6 - Exemplos Numéricos}

Nesse ítem três exemplos de placas serão analisados com o intuito de mostrar o comportamento da formulaçāo empregada. Para cada exemplo serão apresentados dois gráficos. O primeiro mostra os valores adimensionais dos deslocamentos, medidos em pontos que vão do contorno ao centro da placa, ao longo de a até b. O segundo gráfico mostra os valores, também adimensionais, das reações normais, medidas em pontos do contorno, ao longo de $\mathbf{c}$ até $\mathbf{d}$.

Os detalhes das discretizações adotadas estão mostrados nas figuras representativas de cada exemplo. Em todos os casos analisados as placas são simplesmente apoiadas e os carregamento são uniformemente distribuídos. Os dados empregados nos exemplos são:

$$
\begin{array}{ll}
E=10000000 . & \\
h=0.5 & \\
v=0.3 & \text { (espessura da placa) } \\
q=300 . & \text { (carregamento distribuído) }
\end{array}
$$

Para mostra a eficiência do método, os resultados de deslocamentos são comparados com os obtidos em TIMOSHENKO (1968). Pela dificuldade em se diferenciar os dois resultados nos gráficos, a comparação dos valores de deslocamento é apresentada em tabelas, logo abaixo dos respectivos gráficos. 
exemplo 1 - Placa quadrada

$11=12=10$.

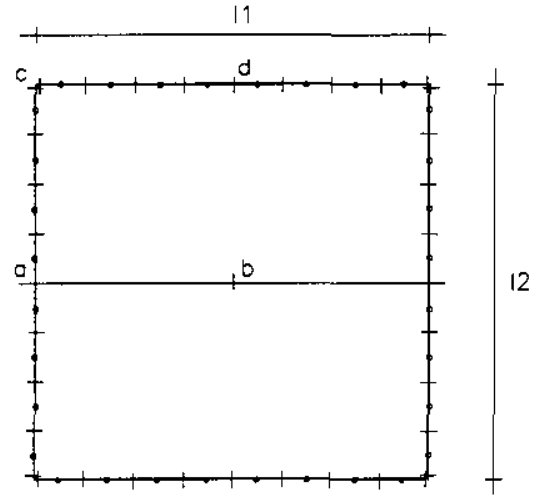

FIGURA 11

\begin{tabular}{|c|c|c|c|c|}
\hline$x(m)$ & 0.50 & 1.00 & 3.00 & 5.00 \\
\hline BEM & 0.071 & 0.129 & 0.165 & 0.177 \\
\hline TIMOSHENKO & 0.0709 & 0.128 & 0.1649 & 0.177 \\
\hline
\end{tabular}

TABELA 1 - Valores do deslocamento vertical [ w.E.h / (11.12.P) ]

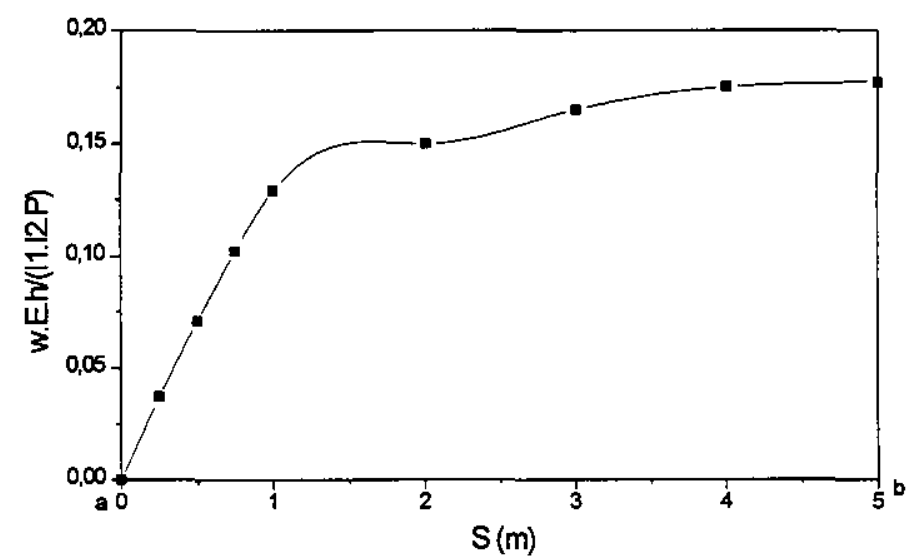

GRÁFICO 1 - Deslocamento vertical ao longo de a-b 


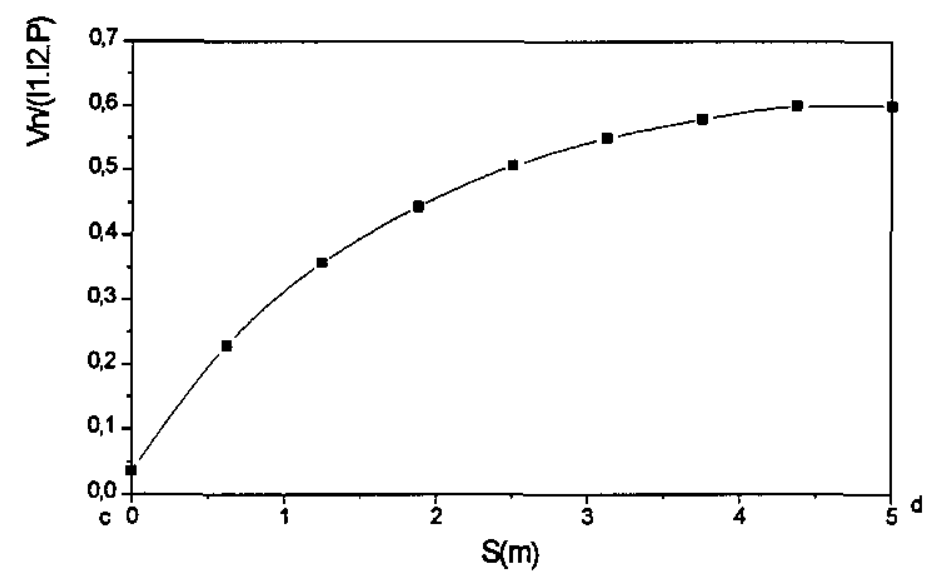

GRÁFICO 2 - Reação normal ao longo do contorno (c-d)

exemplo 2 - Placa retangular

$$
11=8 . \quad 12=4
$$

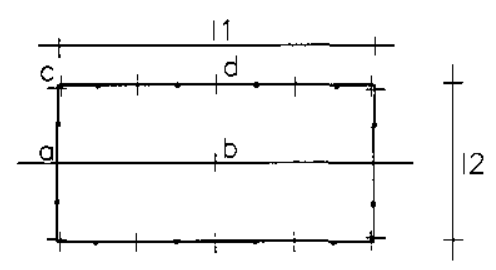

FIGURA 12

\begin{tabular}{|c|c|c|c|c|}
\hline$x(\mathrm{~m})$ & 0.50 & 1.00 & 3.00 & 4.00 \\
\hline BEM & 0.026 & 0.50 & 0.105 & 0.111 \\
\hline TIMOSHENKO & 0.0264 & 0.50 & 0.1046 & 0.1106 \\
\hline
\end{tabular}

TABELA 2 - Valores do deslocamento vertical [ w.E.h / (11.l2.P)] 


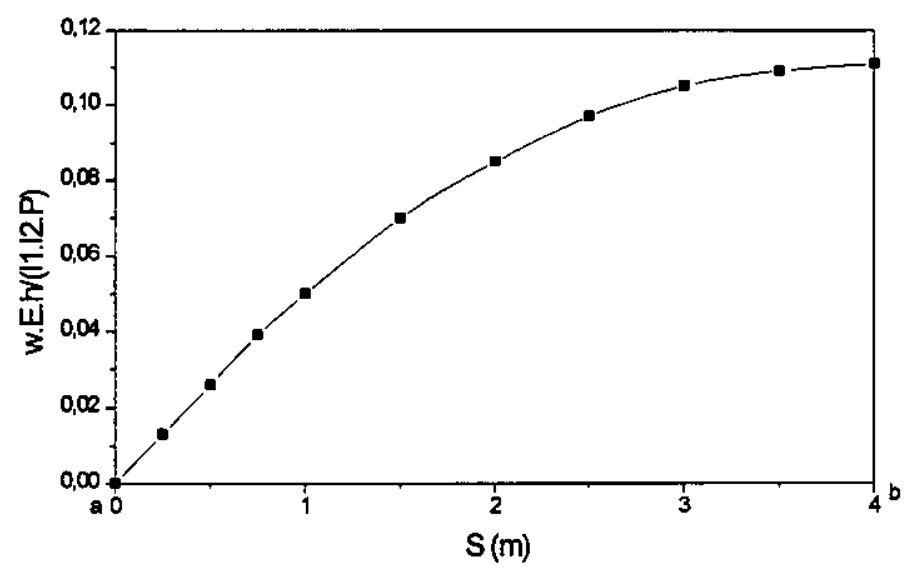

GRÁFICO 3 - Deslocamento vertical ao longo de a-b

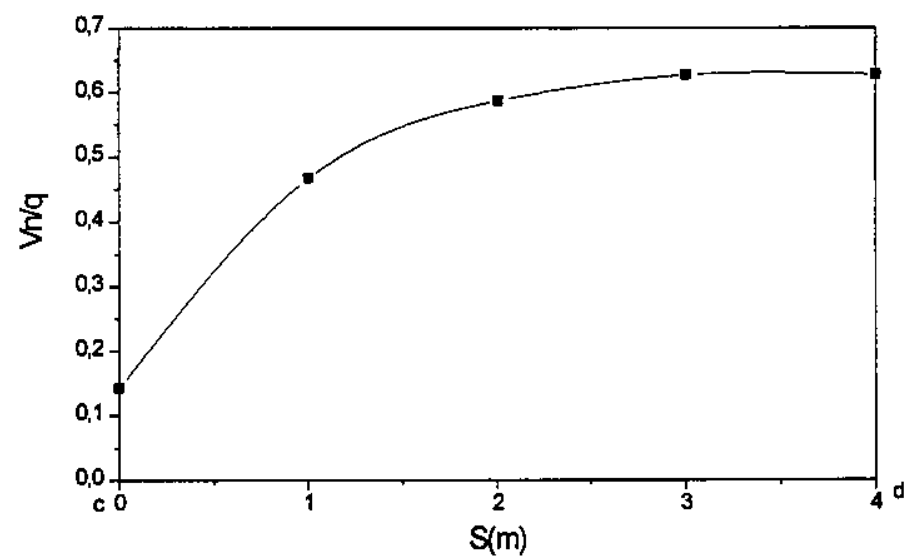

GRÁFICO 4 - Reação normal ao longo do contorno (c-d) 
exemplo 3 - Placa retangular

$$
11=16 . \quad 12=4 .
$$

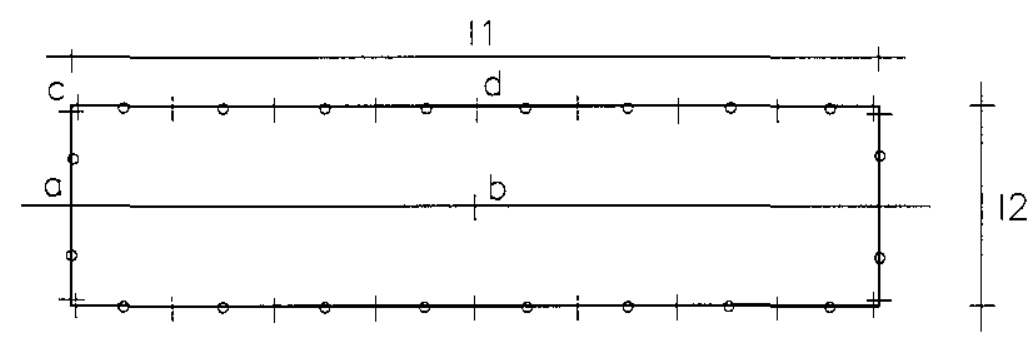

FIGURA 13

\begin{tabular}{|c|c|c|c|c|}
\hline $\mathrm{x}(\mathrm{m})$ & 0.50 & 1.00 & 4.00 & 8.00 \\
\hline BEM & 0.007 & 0.013 & 0.032 & 0.035 \\
\hline TIMOSHENKO & 0.0068 & 0.0129 & 0.0316 & 0.035 \\
\hline
\end{tabular}

TABELA 3 - Valores do deslocamento vertical [ w.E.h / (11.I2.P)]

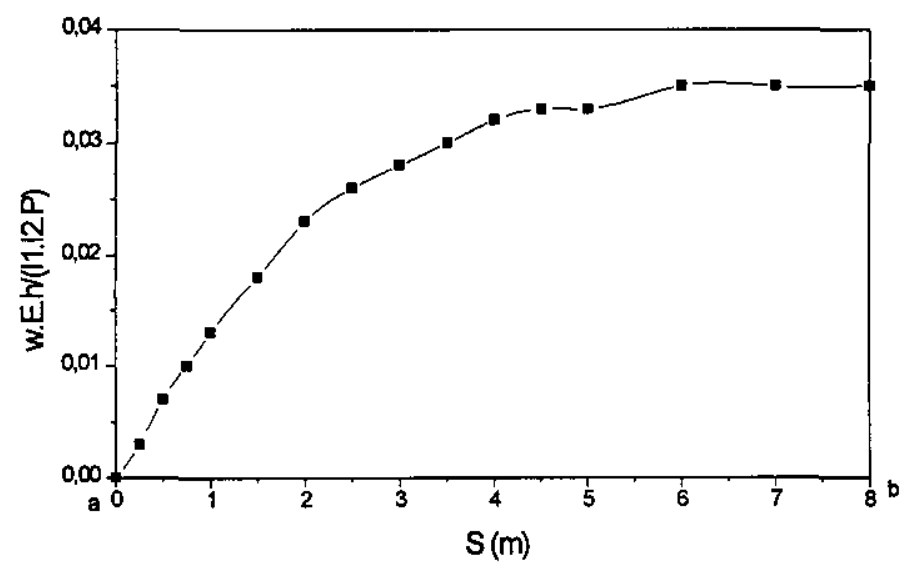

GRÁFICO 5 - Deslocamento vertical ao longo de a-b 


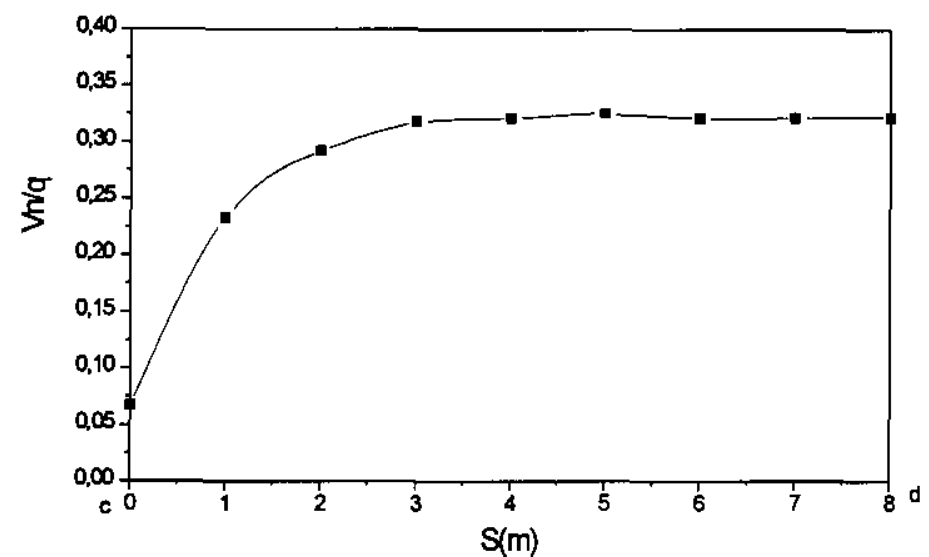

GRÁFICO 6 - Reação normal ao longo do contorno (c-d) 


\section{CAPÍTULO III}

\section{MÉTODO DOS ELEMENTOS DE CONTORNO APLICADO A PLACAS}

\section{- ELASTODINÂMICA -}

\section{1 - Introdução}

O tratamento elastodinâmico de placas foi feito utilizando-se do conceito de "matriz de massa", e foi adotado para este trabalho devido ao desempenho satisfatório que apresenta. Essa forma de tratar problemas elastodinâmicos consiste em incluir o termo inercial nas equaçōes integrais. Essa inclusão se dá em forma de uma integral de domínio, cuja resolução leva a uma matriz que é chamada de "matriz de massa" $\boldsymbol{M}$.

A solução fundamental aqui empregada é a mesma do capítulo anterior (II.34) e, uma vez montadas as matrizes, a resolução do novo sistema de equações é feita através do método da integração direta, utilizando-se do algoritmo de Newmark.

Desta técnica adotada, destacam-se as vantagens dela ser extremamente simples de ser empregada e de proporcionar uma enorme 
economia em termos de tempo de execução computacional, em se tratando de análise dinâmica. A desvantagem principal se refere ao fato da integral de domínio do termo inercial exigir uma discretizaçāo do domínio do problema.

Nesse capítulo o desenvolvimento das integrais é similar ao feito no capítulo anterior, a menos da parcela inercial. Desta forma serão obtidas as mesmas matrizes do capítulo, mais a matriz $\boldsymbol{M}$. Assim sendo, apenas o desenvolvimento odotada para a obtenção desta parcela vai ser detalhado, repetindo-se aqui os termos similares ao capítulo anterior.

\section{2 - Equação integral elastodinâmica}

No capítulo anterior, a equação de equilíbrio (estático) da placa está apresentada em II.3 da seguinte forma:

$$
\frac{\partial^{2} m_{x}}{\partial x^{2}}+\frac{\partial^{2} m_{y}}{\partial y^{2}}+2 \frac{\partial^{2} m_{x y}}{\partial x \partial y}=-g
$$

e, lembrando-se de II.9 ela pode ser reescrita como sendo:

$$
D \Delta \Delta w=g
$$

Quando se pensa no caso de dinâmica de placas, a equação acima passa a ter um termo inercial, função da aceleração, e é chamada de equação de movimento da placa. Essa equação é escrita como se segue,

$$
D \Delta \Delta w+\rho \ddot{w}=g
$$


onde o símbolo $\rho$ corresponde à densidade de massa, e a presença do trema sobre o deslocamento representa a segunda derivada deste em relação ao tempo.

Observando-se agora a equação integral II.58 nota-se a presença do termo de carregamento $g$. Este termo está contido na integral de domínio correspondente à última parcela da equação 11.58 e foi assim escrito substituindo-se a relação II.3 no último termo de II.51. Se, ao invés de II.3, a equação 11.51 receber a equação de movimento III.3, a equação integral da placa passa a ser também dependente de um termo inercial.

Inicialmente tém-se que

$$
\int_{\Omega}\left[\frac{\partial^{2} m_{x}}{\partial x^{2}}+\frac{\partial^{2} m_{y}}{\partial y^{2}}+2 \frac{\partial^{2} m_{x y}}{\partial x \partial y}\right] w^{*} d \Omega=\int_{\Omega}[g-\rho \ddot{w}] w^{*} d \Omega
$$

Desta forma a equação integral 11.58 passa a ser escrita como se segue:

$$
\begin{gathered}
\int_{\Gamma}\left[V_{n}^{*}(q, p) w(p)-m_{n}^{*}(q, p) \frac{\partial w(p)}{\partial n}\right] d \Gamma_{(p)}+\sum_{i=1}^{N c} R_{c_{i}}^{*}(q, p) w_{c_{i}}(p) \\
+\int_{\Omega_{g}} g^{*}(q, p) w(p) d \Omega_{g_{(p)}}=\int\left[V_{n}(p) w^{*}(q, p)-m_{n}(p) \frac{\partial w^{*}(q, p)}{\partial n}\right] d \Gamma_{(p)} \\
+\sum_{i=1}^{N c} R_{c_{i}}(p) w_{c_{i}}^{*}(q, p)+\int_{\Omega_{g}} g(p) w^{*}(q, p) d \Omega_{g_{(p)}}-\rho \int_{\Omega_{g}} \ddot{w}(p, t) w^{*}(q, p) d \Omega_{g_{(p, t)}}
\end{gathered}
$$


A integração da última parcela da equação anterior é que vai dar origem á matriz $\boldsymbol{M}$, que costuma ser chamada de "matriz de massa". Um outro aspecto que se deve observar é que as variáveis nodais passam também a serem escritas em função do tempo. A partir de II.5, e recordando-se o que foi feito no capítulo anterior, escreve-se o sistema matricial para um problema elastodinâmico.

$$
\underset{\sim}{H} \underset{\sim}{U}=\underset{\sim}{G} \underset{\sim}{P}-\underset{\sim}{M} \underset{\sim}{\ddot{U}}+\underset{\sim}{F}
$$

\section{3 - Montagem da matriz de massa $M$}

Conforme já foi dito anteriormente, matriz $\boldsymbol{M}$ é gerada a partir da integral de domínio

$$
\rho \int_{\Omega_{g}} \ddot{w}(p, t) w^{*}(q, p) d \Omega_{g_{(p, t)}}
$$

Seguindo-se a mesma forma de resolução das integrais de contorno, apresentada no capitulo anterior, a integral III.7 também será resolvida numericamente.

A forma adotada para integrar numericamente III.7 é a de utilizarse da técnica de integração por células. Esta técnica consiste em subdividir o domínio a ser analisado em pequenas superfícies, denominadas células, aplicando-se nestas o método da Quadratura de Gauss para efetuar a integração. Essas células nada mais sāo do que elementos finitos de superfície e, desta forma, existem vários tipos de células as quais diferem entre si pela 
forma e pelo número de nós que as definem. É justamente nessa subdivisão que reside a principal desvantagem do método, por exigir uma discretização do domínio. Essa discretização, por sua vez, depende do tipo de célula adotada, uma vez que será necessária a colocação de pontos internos para defini-la.

Da mesma forma que no caso da integração numérica no contorno, obtém-se valores que estão referidos aos nós que definem as células. Para tanto utilizam-se de funções interpoladoras, caracteristicas do tipo de célula adotada, que neste caso estão em função de duas coordenadas adimensionais $\xi$ e $\eta$. Neste trabalho optou-se pela integração utilizando-se uma célula quadrilátera de 8 nós, conforme figura 11, mostrada a seguir,
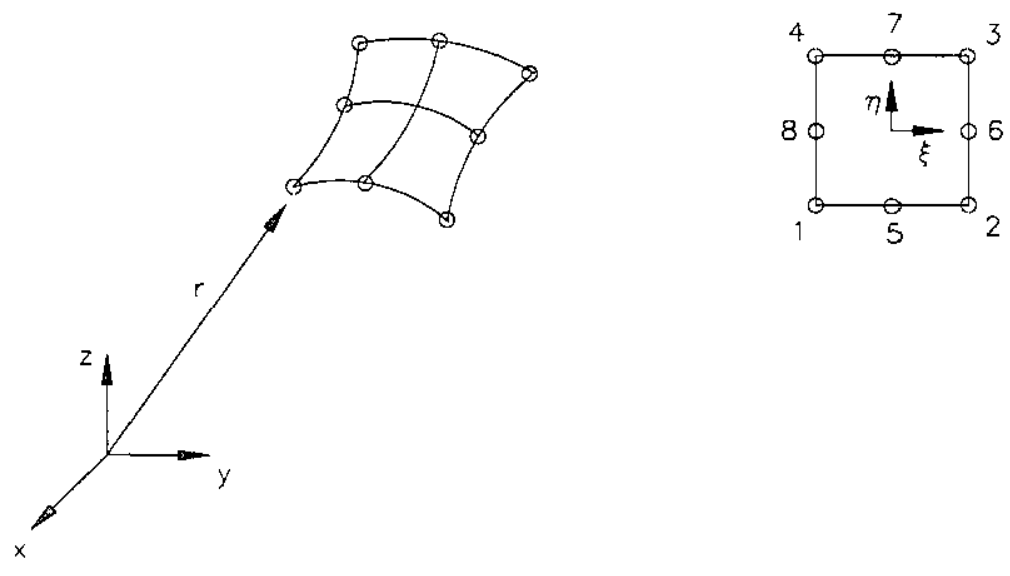

FIGURA 11

Os detalhes da integração numérica de III.7 estão apresentados no apêndice $\mathbf{A}$, incluindo-se a determinação do Jacobiano da célula quadrilátera adotada, cujas funçōes são: 


$$
\begin{aligned}
& \phi_{1}=\frac{1}{4}(1-\xi)(1-\eta)(-\xi-\eta-1) \\
& \phi_{2}=\frac{1}{4}(1+\xi)(1-\eta)(\xi-\eta-1) \\
& \phi_{3}=\frac{1}{4}(1+\xi)(1+\eta)(\xi+\eta-1) \\
& \phi_{4}=\frac{1}{4}(1-\xi)(1+\eta)(-\xi+\eta-1) \\
& \phi_{5}=\frac{1}{2}\left(1-\xi^{2}\right)(1-\eta) \\
& \phi_{6}=\frac{1}{2}\left(1-\eta^{2}\right)(1+\xi) \\
& \phi_{7}=\frac{1}{2}\left(1-\xi^{2}\right)(1+\eta) \\
& \phi_{8}=\frac{1}{2}\left(1-\eta^{2}\right)(1-\xi)
\end{aligned}
$$

Uma vez efetuada a integração em todo o domínio da placa, obtém-se a matriz $\boldsymbol{M}$. Deve-se notar, porém, que existirá uma diferença no número de colunas entre esta matriz e as matrizes $H \in G$, montadas no capítulo anterior. Nestas matrizes, para cada ponto do contorno, são escritas duas equaçōes, as quais correspondem aos dois pontos colocados externamente ao domínio. Para cada uma destas equações são escritos dois valores nodais que vão formar as matrizes $\boldsymbol{H}$ e $\mathbf{G}$. Estes valores são: o deslocamento e derivada deste com relação à normal, no caso da matriz $\mathbf{G}$, a cortante equivalente e o momento fletor normal, no caso da matriz $\boldsymbol{H}$. Como no caso da matriz $\boldsymbol{M}$ apenas a integral do termo do deslocamento é feita, o número de colunas fica sendo menor. Para tornar a matriz compataivel com o sistema de equações 11.75 , basta relocar as colunas referentes aos nós do contorno em colunas ímpares, criando colunas pares nulas. As colunas referentes aos cantos da placa e aos pontos internos passam a ocupar posições a partir da última coluna nula. Desta 
forma a matriz deixa de ter dimensões NVTT $\times(N N+N C A N+N N I)$ e passa a ter as mesmas dimensões de $\boldsymbol{H}$ e $\boldsymbol{G}$, ou seja, NVTT x NVTT, e o sistema passa a ser compativel.

\section{4 - Solução do sistema dinâmico}

O sistema Ill.9 será resolvido pelo método da integração direta, através do algoritmo de Newmark (WARBURTON (1964)). Neste algoritmo assume-se que os deslocamentos e as velocidades no final de um intervaio de tempo $\left(t_{s+1}\right)$ podem ser expressos em termos dos deslocamentos, das velocidades e das aceleraçōes no início deste intervalo $\left(t_{s}\right)$, e das velocidades no final $\left(t_{S+1}\right)$. Estas duas relações são as seguintes:

$$
\begin{aligned}
& \dot{\sim}_{S+1}^{\dot{U}}={\underset{\sim}{U}}_{S}+\frac{1}{2}(\Delta t)\left[{\underset{\sim}{U}}_{S}+{\underset{\sim}{U}}_{S+1}^{\ddot{U}}\right] \\
& {\underset{\sim}{S+1}}_{U_{S}}={\underset{\sim}{U}}+(\Delta t) \underset{\sim}{\dot{U}}+\left(\frac{1}{2}-\beta\right)(\Delta t)^{2} \underset{\sim}{\ddot{U}}+\beta(\Delta t)^{2}{\underset{\sim}{U}}_{S+1}^{\ddot{U}}
\end{aligned}
$$

O passo seguinte para encontrar o algoritmo de Newmark é escrever-se a equação III.9 para 3 intervalos sucessivos de tempo.

$$
\begin{aligned}
& \underset{\sim}{M} \ddot{\sim}_{S+1}^{\ddot{U}}+\underset{\sim}{H} \sim_{S+1}^{U}=\underset{\sim}{G} \sim_{S+1}^{P}+\sim_{S+1}^{F} \\
& \underset{\sim}{M} \underset{\sim S}{\ddot{U}}+\underset{\sim}{H U}{\underset{\sim}{U}}_{S}=\underset{\sim}{P} \underset{\sim S}{P}+\underset{\sim S}{F} \quad
\end{aligned}
$$




$$
\underset{\sim}{M} \underset{\sim}{\ddot{U}}+\underset{\sim}{H}{\underset{\sim}{S-1}}_{U_{S-1}}=\underset{\sim}{P} \underset{\sim S-1}{F}+{\underset{\sim}{S-1}}^{P}
$$

Efetuando-se agora uma manipulação algébrica nestas 3 equações, incluindo-se a substituição das relações III.12 no resultado obtido, pode-se escrever que:

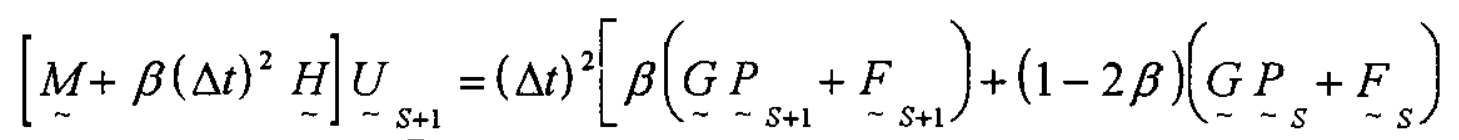

$$
\begin{aligned}
& \left.+\beta\left(\underset{\sim}{G} \underset{\sim}{P}+F_{\sim-1}^{P}\right)\right]+\left[2 \underset{\sim}{M-1}(\Delta t)^{2}(1-2 \beta) \underset{\sim}{H}\right] \underset{\sim}{U}-\left[\underset{\sim}{M}+\beta(\Delta t)^{2} \underset{\sim}{H}\right]_{\sim} \underset{S-1}{U}
\end{aligned}
$$

Com esta equação (III.14) pode-se obter o vetor de deslocamentos no instante $t_{s+1}$, a partir dos valores obtidos nos 2 instantes anteriores ( $t_{s} e t_{s-1}$ ), bem como dos valores conhecidos dos vetores de esforços e carregamento.

Para o caso do primeiro vetor a ser calculado, no primeiro instante, utiliza-se uma outra equação, diferente de III.14, obtida a partir das duas primeiras de III.13. Para este primeiro instante, a equação é:

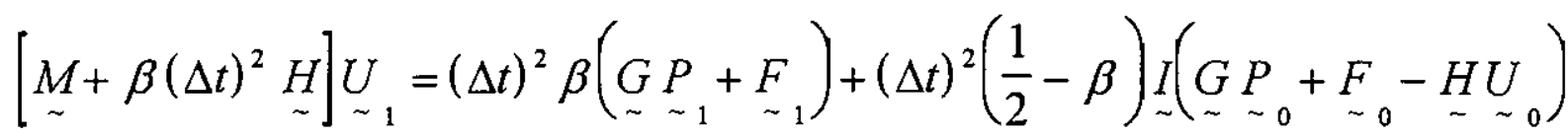

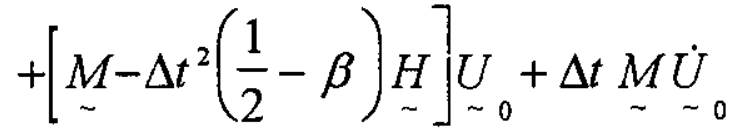




\section{5 - Exemplos numéricos}

Os exemplos apresentados a seguir são de placas retangulares simplesmente apoiadas e submetidas a um carregamento transversal (q) uniformemente distribuído e constante ao longo do tempo de análise. Em todos os exemplos esse tempo total de análise corrsponde a 150 passos de tempo.

Para um mesmo exemplo são apresentados os resultados obtidos para o deslocamento do nó central e para a reação no centro do contorno, variando apenas a discretização do domínio. Isso foi feito para mostrar a versatilidade da formulação, além da acuidade dos resultados, mesmo para discretizações mais "pobres" do dominio.

As propriedades do material e o carregamento aplicado estão descritos abaixo, enquanto que as dimensões das placas e o passo de tempo empregado em cada exemplo são dados no início dos mesmos.

$$
\begin{aligned}
E & =7.8 \times 10^{10} \mathrm{Kg} / \mathrm{m} / \mathrm{s}^{2} \\
v & =0.3 \\
\rho & =2500 \mathrm{Kg} / \mathrm{m}^{3} \\
h & =0.1 \mathrm{~m} \\
q & =300 . \mathrm{Kg}
\end{aligned}
$$




$$
\text { exemplo } 4-\begin{aligned}
11 & =10 . \mathrm{m} \\
12 & =10 . \mathrm{m} \\
\Delta t & =0.01 \mathrm{~s}
\end{aligned}
$$

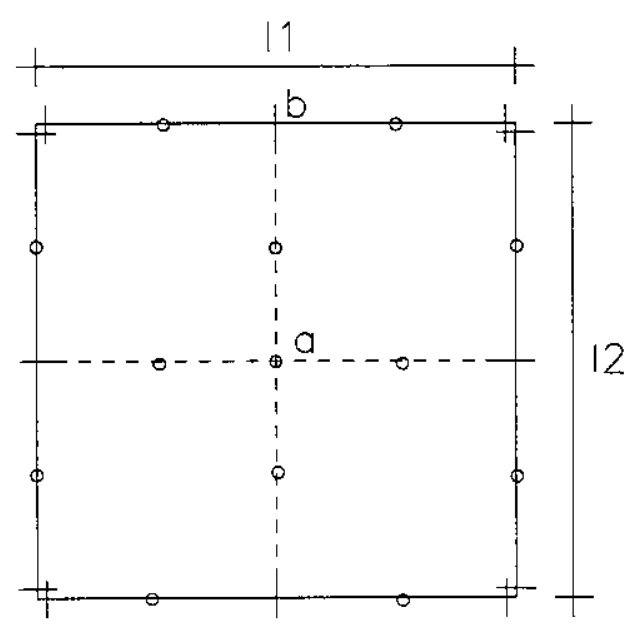

FIGURA 12

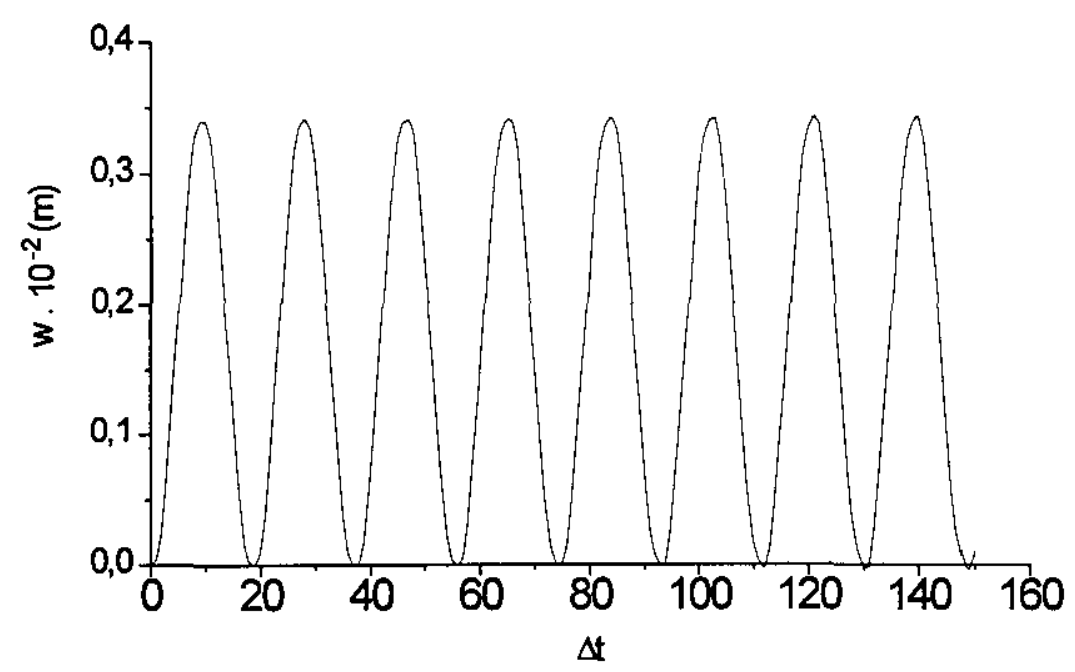

GRÁFICO 7 - variação do deslocamento do nó central (ponto a) 


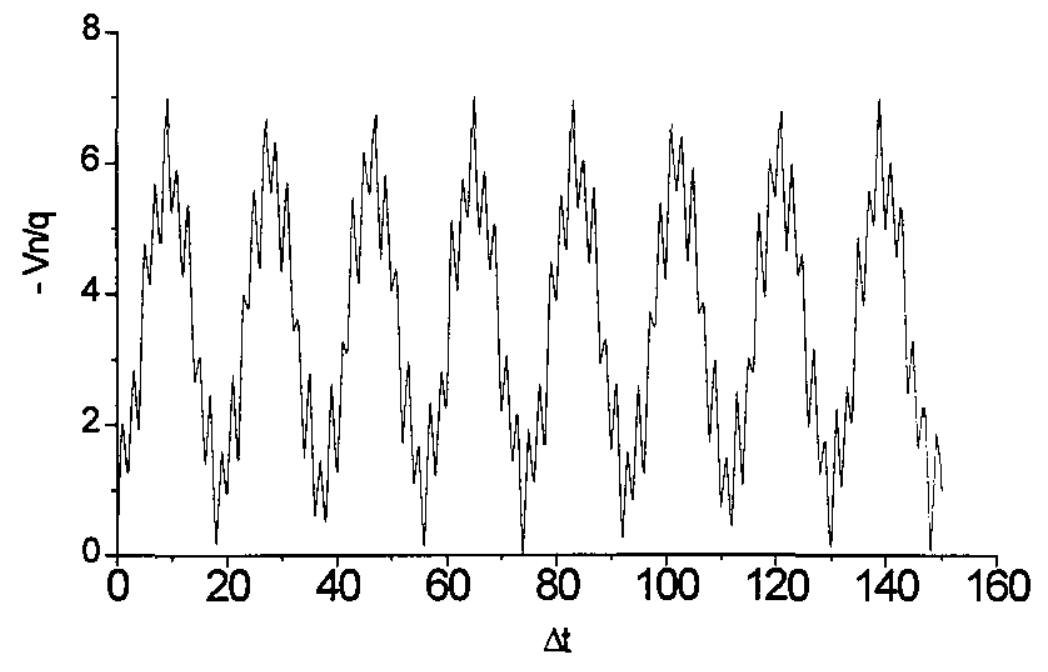

GRAFICO 8 - variação do reação $V_{n}$ (ponto b)

$$
\text { exemplo } 5-\begin{aligned}
11 & =10 . \mathrm{m} \\
12 & =10 . \mathrm{m} \\
\Delta t & =0.01 \mathrm{~s}
\end{aligned}
$$

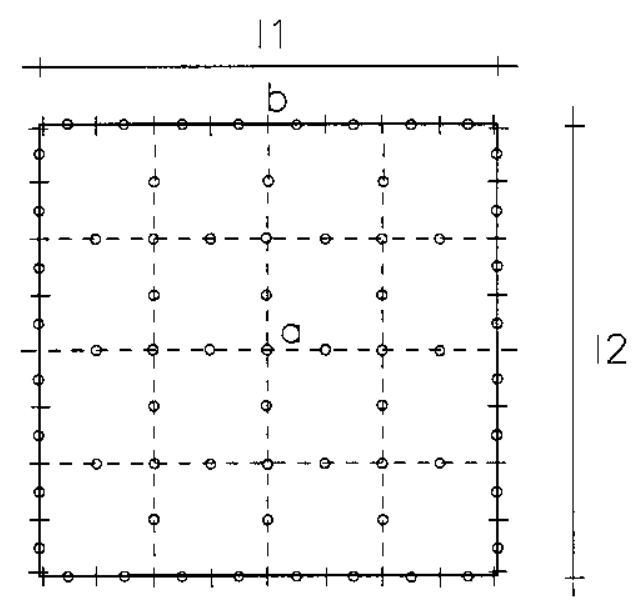

FIGURA 13 


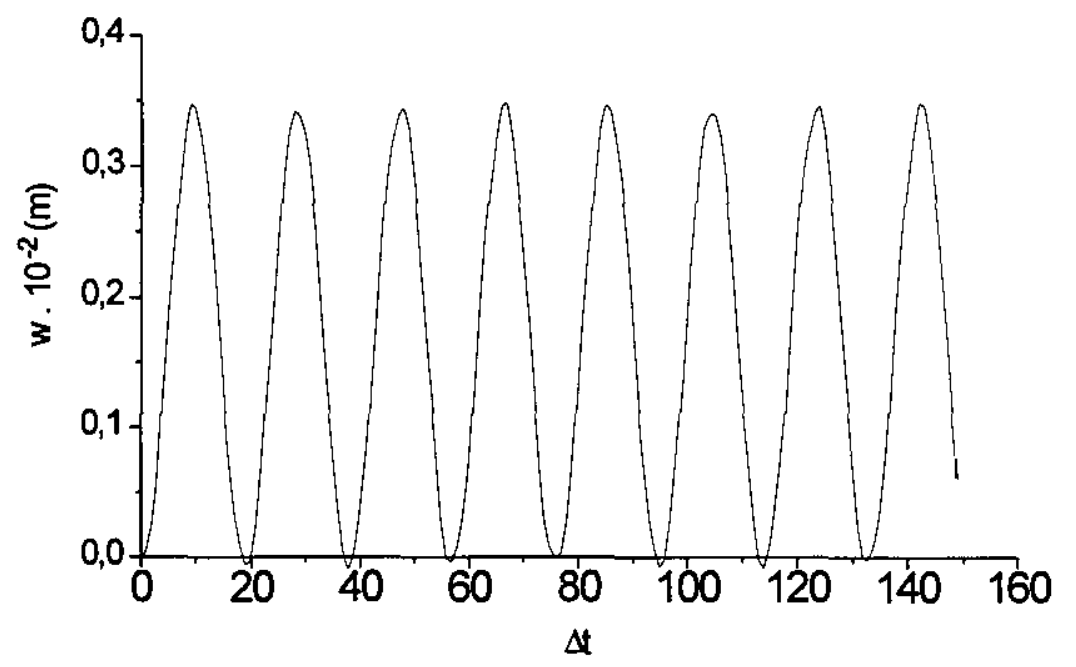

GRÁFICO 9 - variação do deslocamento do nó central (ponto a)

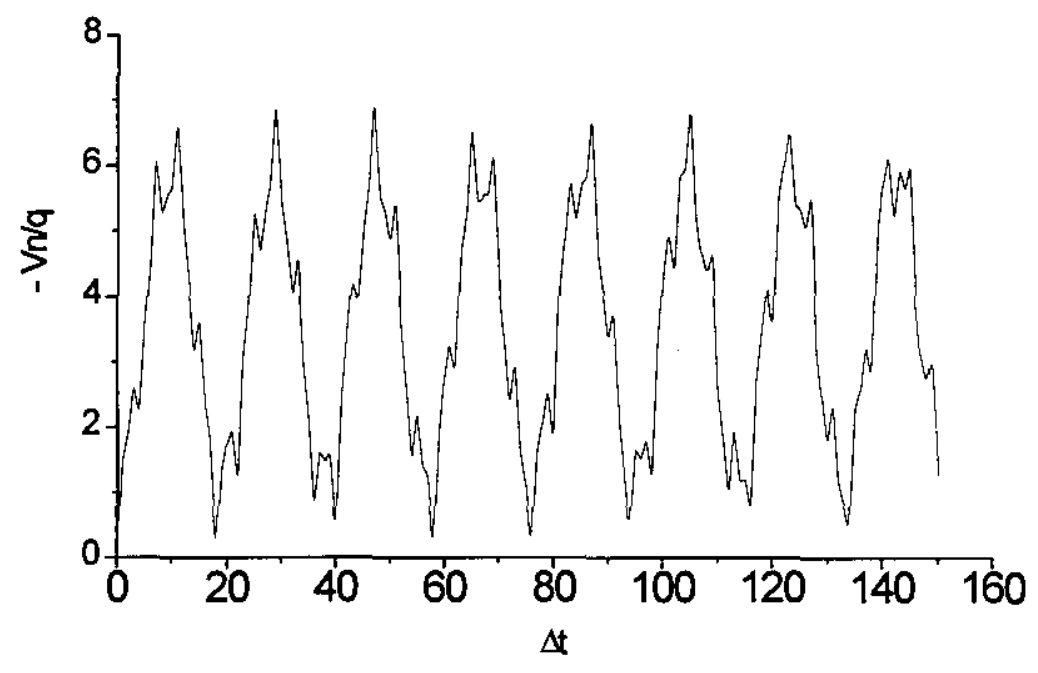

GRÁFICO 10 - variação do reação $V_{n}$ (ponto b) 
exemplo $6-11=8 . \mathrm{m}$

$12=4 . m$

$\Delta \mathrm{t}=0.0025 \mathrm{~s}$

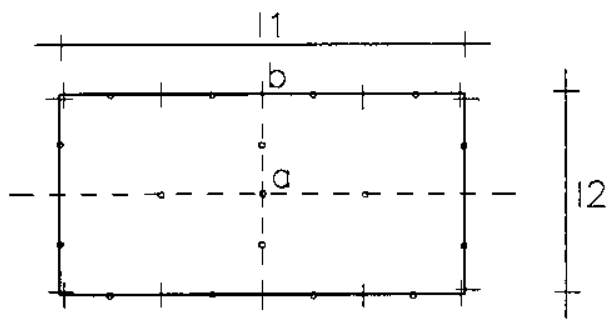

FIGURA 14

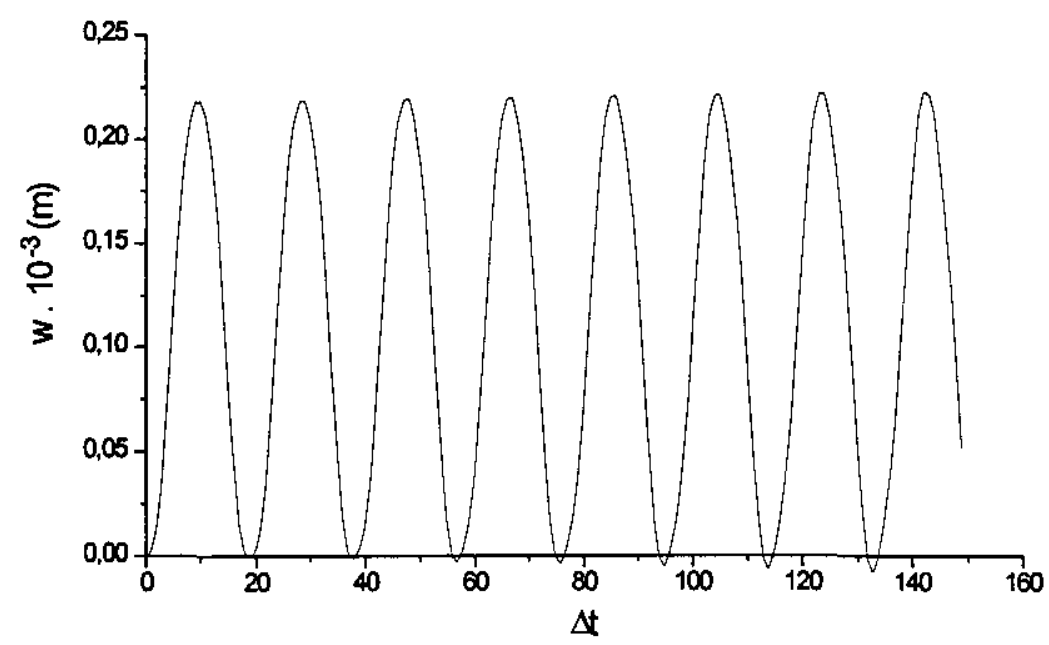

GRÁFICO 11 - variação do deslocamento do nó central (ponto a) 


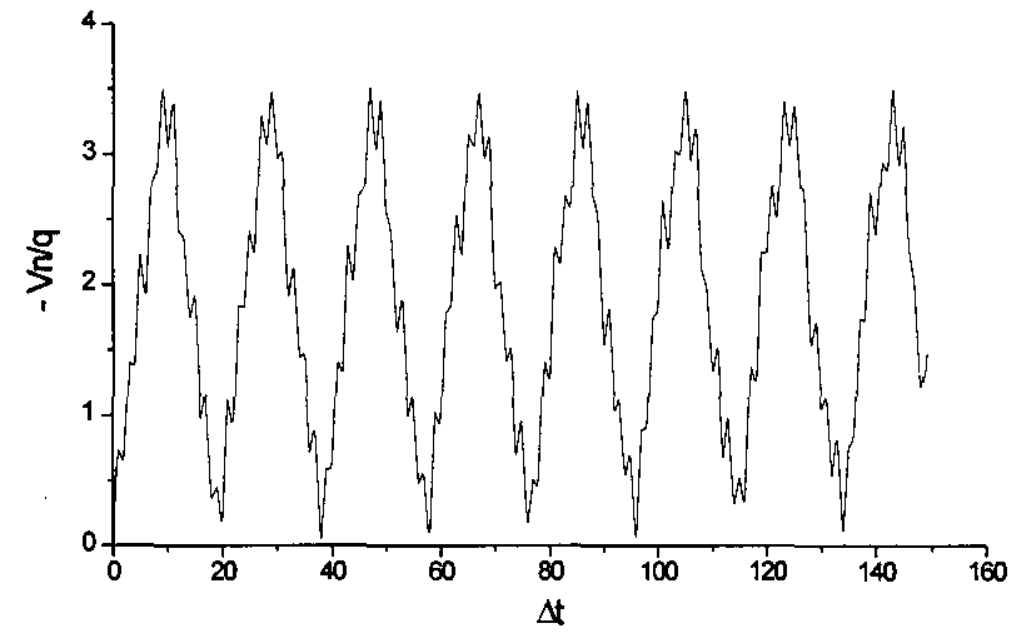

GRÁFICO 12 - variação do reação $V_{n}$ (ponto b)

exemplo $7-11=8 \cdot \mathrm{m}$

$12=4 . m$

$\Delta \mathrm{t}=0.0025 \mathrm{~s}$

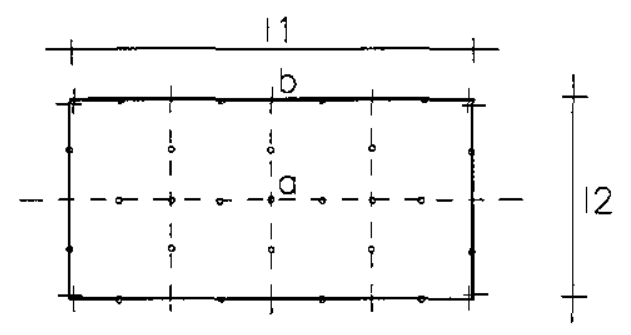

FIGURA 15 


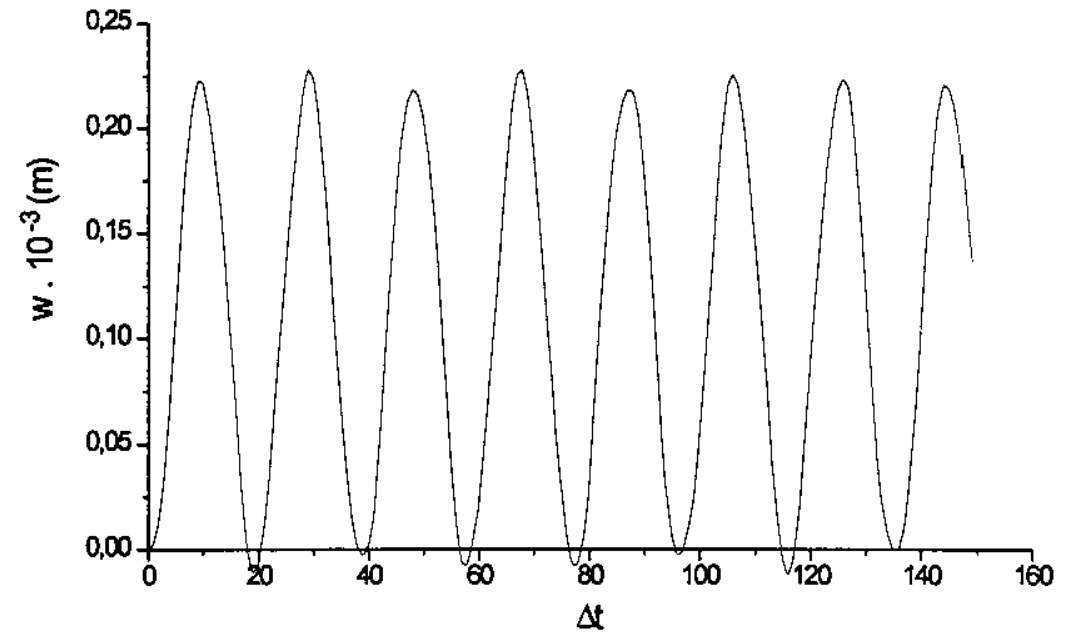

GRÁFICO 13 - variação do deslocamento do nó central (ponto a)

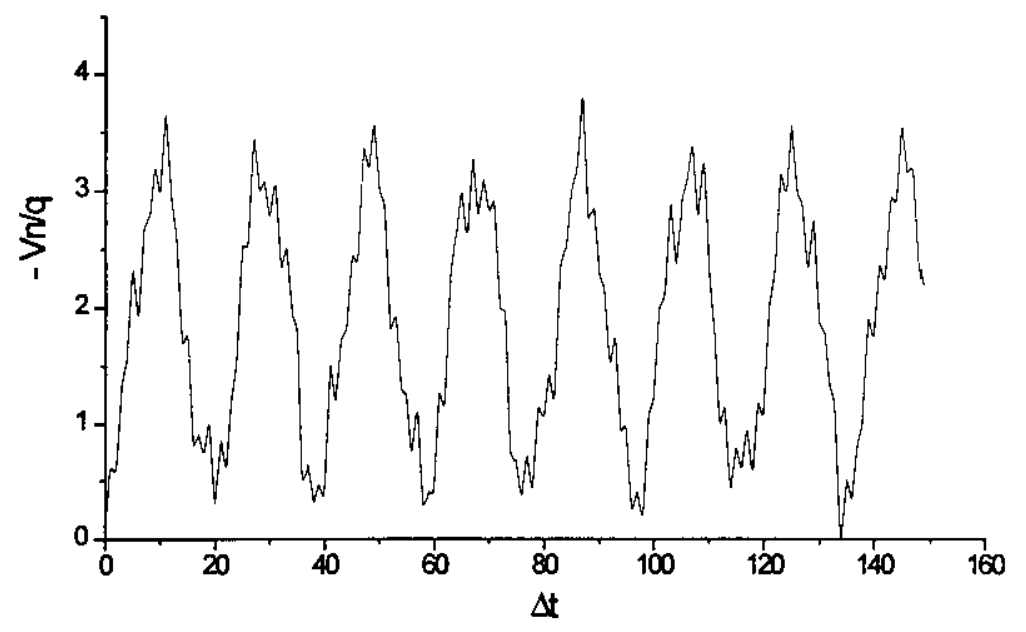

GRÁFICO 14 - variação do reação $V_{n}$ (ponto b) 
exemplo $8-11=16 . \mathrm{m}$

$12=4 . m$

$\Delta t=0.0033 \mathrm{~s}$

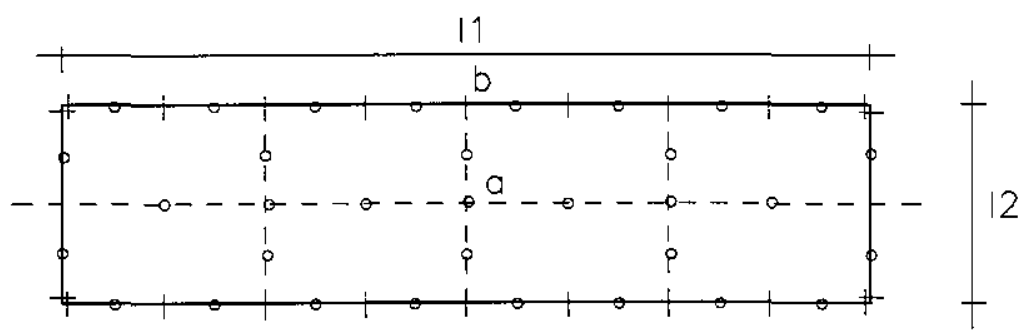

FIGURA 16

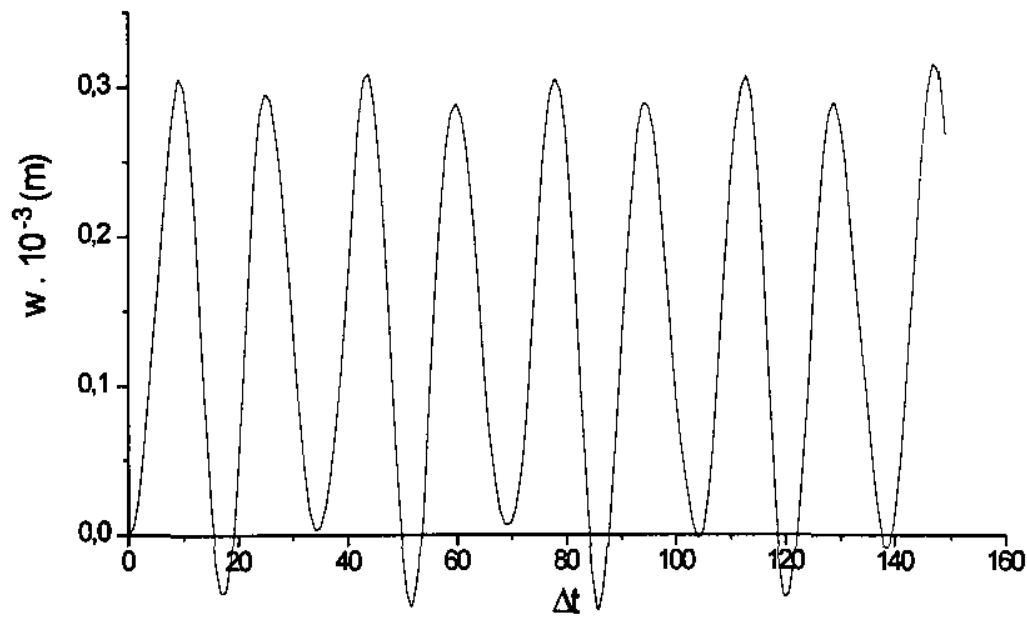

GRÁFICO 15 - variação do deslocamento do nó central (ponto a) 


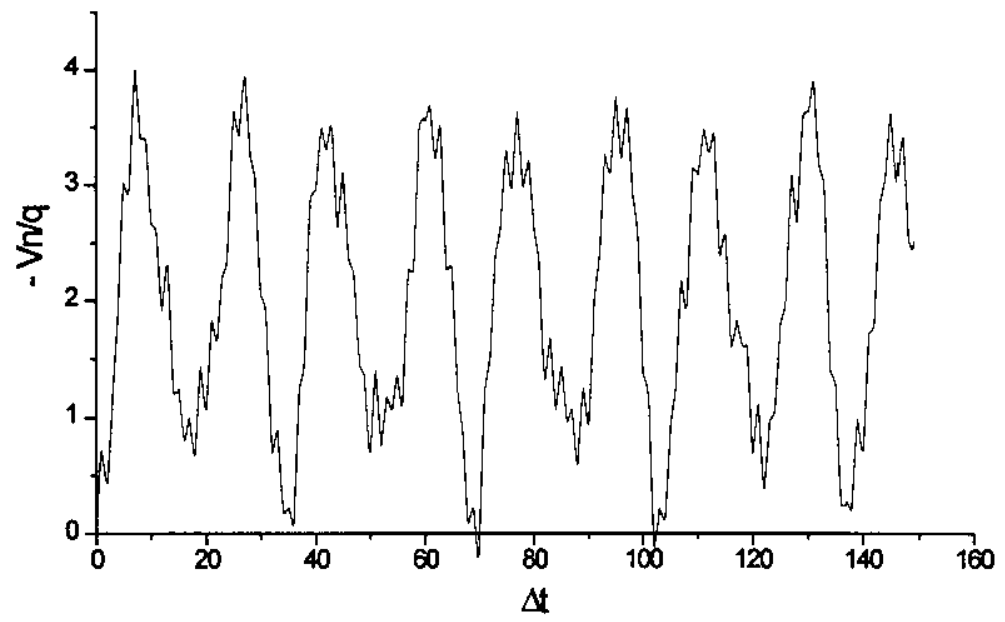

GRÁFICO 16 - variação do reação $V_{n}$ (ponto b)

exemplo $9-11=16 . \mathrm{m}$

$12=4 . m$

$\Delta \mathrm{t}=0.0033 \mathrm{~s}$

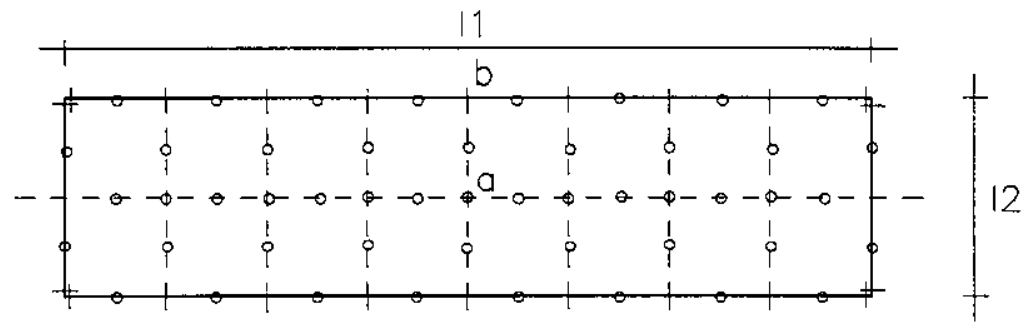

FIGURA 17 


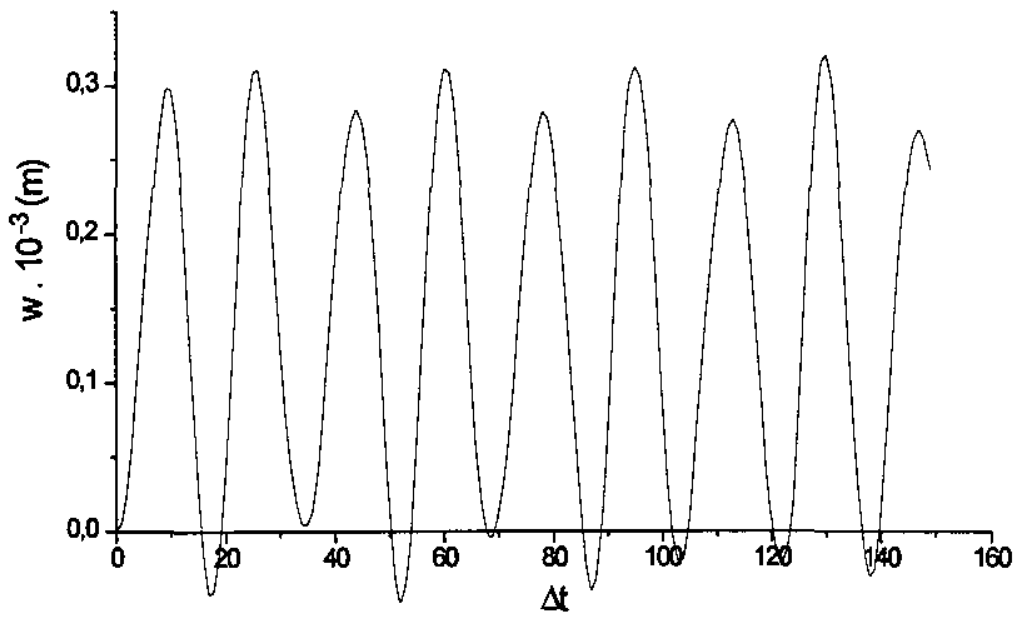

GRÁFICO 17 - variação do deslocamento do nó central (ponto a)

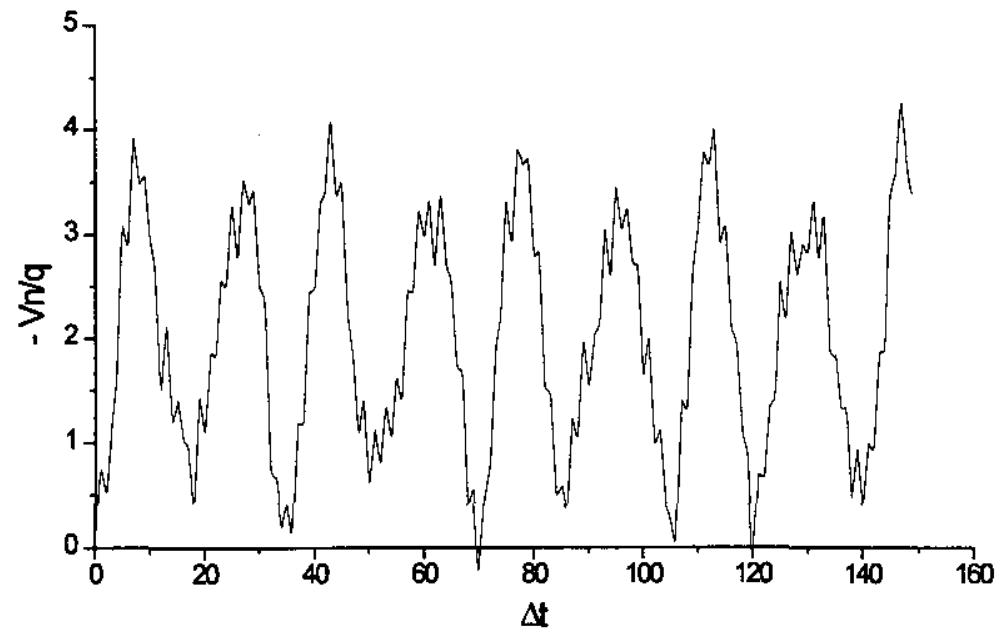

GRÁFICO 18 - variação do reação $V_{n}$ (ponto b) 


\section{CAPÍTULO IV}

\section{ANÁLISE DA ASSOCIAÇÃO PLACA-SOLO ATRAVÉS DAS FORMULAÇÕES DO MEC}

\section{1 - Introdução}

Nos capitulos anteriores foram descritas as formulações para as análises elastostática e elastodinâmica de placas, via BEM. Neste capítulo será apresentada a implementaçāo da formulação descrita nos capítulos anteriores, agora analisando a interação da placa com o solo.

Conforme já foi comentado no capítulo I, a formulação do MEC se adequa convenientemente à análise de meios considerados ilimitados, como é o caso do solo. No caso da análise de placas associadas ao solo, a formulação mais utilizada no estudo de problemas da engenharia é a obtida com o uso do Método dos Elementos Finitos. Contudo se pretende aqui apresentar o comportamento da associação entre os dois meios (placa-solo) utilizando-se apenas o Método dso Elementos de Contorno.

A formulação do MEC adotada para a análise do solo é a apresentada no trabalho de CODA (1993). Para o acoplamento da placa com o solo foi utilizada a implementação numérica resultante do referido trabalho. 
A associação da placa com o solo se dá através de uma adequação dos graus de liberdade das duas estruturas, já que são estruturas tratadas em espaços bi e tridimensionais, respectivamente. Uma vez feita esta adequação, utiliza-se da técnica de sub-regiões [VENTURINI (1984), CODA (1993)] para proceder à análise do sistema acoplado.

Ao final do capítulo alguns exemplos são apresentados, cujos resultados são comparados com os obtidos nos trabalhos de CODA (1993) e PAIVA (1994). No caso do primeiro, a placa é tratada via FEM utilizando-se de um elemento tipo LCCT9 (Linear Curvature Compatible Triangle), desenvolvido por CLOUGH e FELIPPA (1968). No trabalho de PAIVA (1994) a placa é tratada via BEM, levando-se em conta apenas o movimento vertical do sistema acoplado. Neste trabalho, tanto as forças de atrito quanto os efeitos da expansāo do material são desconsiderados.

Inicialmente será feita uma comparação entre os resultados obtidos através do uso de cada uma das três formulações, para o caso da análise estática de uma placa quadrada, submetida a um carregamento uniformemente distribuído. A partir desta comparação inicial, outros exemplos, tanto estáticos como dinâmicos, sāo apresentados, com os resultados obtidos com o uso da formulação apresentada neste trabalho, sempre comparados aos obtidos pela formulação apresentada por CODA (1993).

\section{2 - Tratamento do meio tridimensional (solo)}

Conforme já foi mencionado na introdução, apenas a implementação numérica do trabalho desenvolvido por CODA (1993) foi utilizada. Como o autor se utiliza da notação indicial, e como as expressões 
serão transcritas diretamente do referido trabalho, torna-se conveniente apresentar-se algumas convenções desta notação indicial, bem como as relações entre tensões, deformações e deslocamentos do estado plano.

\section{i - Convenção de somatório}

A repetição de um índice num termo indica a soma de todas as componentes com respeito a este índice, em toda a sua variação, ou seja:

$$
\begin{aligned}
& a_{i i}=a_{11}+a_{22}+a_{33} \\
& a_{i j} b_{i}=a_{1 j} b_{1}+a_{2 j} b_{2}+a_{3 j} b_{3}
\end{aligned}
$$

ii - Convenção para derivadas parciais

A virgula acompanhada por índices representa a derivada parcial com relação às coordenadas referidas a estes, ou seja:

$$
\begin{aligned}
& \frac{\partial f_{i}}{\partial x_{j}}=f_{i, j} \\
& \frac{\partial^{2} f_{k}}{\partial x_{i} \partial x_{j}}=f_{k, i j}
\end{aligned}
$$

iii - Delta de Kronecker $\left(\delta_{i j}\right)$

É um parâmetro auxiliar que assume os seguintes valores:

$$
\begin{array}{lll}
\delta_{i j}=0 & \text { se } & i \neq j \\
\delta_{i j}=1 & \text { se } & i=j
\end{array}
$$


As relações entre tensões, deformações e deslocamentos são as mesmas tanto no caso estático como no dinâmico, e são as seguintes:

$$
\begin{aligned}
& \varepsilon_{i j}=\frac{1}{2}\left(u_{i, j}+u_{j, i}\right) \\
& \sigma_{i j}=\lambda u_{k, k} \delta_{i j}+G\left(u_{j, i}+u_{i, j}\right)
\end{aligned}
$$

onde $\mathrm{G}$ (já definida anteriormente) e $\lambda$ são chamadas constantes de Lamé. Esta última é expressa por:

$$
\lambda=\frac{2 G v}{1-2 v}
$$

\subsection{1 - Soluções fundamentais}

Seja um corpo isotrópico que exibe um comportamento elástico linear. As equações diferenciais que governam o seu equilíbrio estático e dinâmico, respectivamente, são conhecidas como equações de Navier-Cauchy escritas da seguinte forma:

$$
\begin{aligned}
& G u_{i, j j}+(\lambda+G) u_{j, j i}+b_{i}=0 \\
& \left(C_{1}^{2}-C_{2}^{2}\right) u_{j, j i}+C_{2}^{2} u_{i, j j}+b_{i} / \rho=\ddot{u}_{i}
\end{aligned}
$$


onde $b_{i}$ representa as forças de volume, $\rho$ é a densidade do meio, e $C_{1}$ e $C_{2}$ são as velocidades de propagação longitudinal e transversal, respectivamente. Nas duas expressões $i$ e $j$ variam de 1 a 3.

A solução fundamental empregada para o caso elastostático é devido a KELVIN (1962) e representa fisicamente o efeito de uma carga unitária estática atuando em um domínio infinito. Para obtê-la basta substituir as forças de volume $b_{i}$ da equação IV.7 pela função Delta de Dirac $\delta(s, q)$. O resultado é:

$$
u_{k i}^{*}(s, q)=\frac{1}{16 \pi(1-v) G r}\left\{(3-4 v) \delta_{k i}+r_{, k} r_{, i}\right\}
$$

onde $k$ corresponde à direção da aplicação da carga unitária em $s, i$ à direção do deslocamento gerado em $q, r$ é a distância entre os pontos $s$ e $q$, e $\delta_{\mathrm{ki}}$ representa o Delta de Kronecker.

No caso elastodinâmico é usual tomar-se um caso particular do estado de Stokes como solução fundamental. A forma usual para se encontrar a solução fundamental é substituir o termo da força de volume da eq. IV.8 pela carga concentrada, função do tempo, dada por:

$$
b_{k i}^{*}=f(\tau) \delta(q-s) \delta_{k i}
$$

onde $f(\tau)$ é uma função de carregamento dependente do tempo. Uma vez substituido IV.10 em IV.8, e integrando-se a equação resultante, obtém-se a solução fundamental de Stokes, dada por:

$$
u_{k i}^{*}(q, \tau ; s / f)=\frac{1}{4 \pi \rho}\left\{\left(\frac{3 r_{i} r_{k}}{r^{3}}-\frac{\delta_{k t}}{r}\right) \int_{C_{1}^{\prime}}^{C_{2}^{\prime}} \frac{d}{1}(\tau-\alpha r) d \alpha+\frac{r_{i} r_{k}}{r^{3}}\left[\frac{1}{C_{1}^{2}} f\left(\tau-\frac{r}{C_{1}}\right)\right.\right.
$$




$$
\left.\left.-\frac{1}{C_{2}^{2}} f\left(\tau-\frac{r}{C_{2}}\right)\right]+\frac{\delta_{k i}}{r C_{2}^{2}} f\left(\tau-\frac{r}{C_{2}}\right)\right\}
$$

A partir da expressão de u" pode-se obter a expressão para a tração p*, que fica da forma:

$$
\begin{aligned}
p_{k i}^{*}(q, \tau ; s / f) & =\frac{n_{j}}{4 \pi}\left\{-6 C_{2}^{2}\left[5 \frac{r_{i} r_{j} r_{k}}{r^{5}}-\frac{\delta_{i j} r_{k}+\delta_{k i} r_{j}+\delta_{j k} r_{i}}{r^{3}}\right] \int_{C_{1}^{\prime}}^{C_{2}^{-1}} \alpha f(\tau-\alpha r) d \alpha+2\left[6 \frac{r_{i} r_{j} r_{k}}{r^{5}}\right.\right. \\
& \left.-\frac{\delta_{k i} r_{j}+\delta_{i j} r_{k}+\delta_{j k} r_{i}}{r^{3}}\right]\left[f\left(\tau-\frac{r}{C_{2}}\right)-\frac{C_{2}^{2}}{C_{1}^{2}} f\left(\tau-\frac{r}{C_{1}}\right)\right]+2 \frac{r_{i} r_{j} r_{k}}{r^{4} C_{2}}\left[\dot{f}\left(\tau-\frac{r}{C_{2}}\right)\right. \\
& \left.+\frac{C_{2}^{3}}{C_{1}^{3}} \dot{f}\left(\tau-\frac{r}{C_{1}}\right)\right]-\frac{r_{k} \delta_{i j}}{r^{3}}\left(1-2 \frac{C_{2}^{2}}{C_{1}^{2}}\right)\left[f\left(\tau-\frac{r}{C_{1}}\right)+\frac{r}{C_{1}} \dot{f}\left(\tau-\frac{r}{C_{1}}\right)\right] \\
& \left.-\frac{\delta_{k i} r_{j}+\delta_{j k} r_{i}}{r^{3}}\left[f\left(\tau-\frac{r}{C_{2}}\right)+\frac{r}{C_{2}} \dot{f}\left(\tau-\frac{r}{C_{2}}\right)\right]\right\}
\end{aligned}
$$

Essa solução pode ser derivada aplicando nela uma apropriada função $f(\tau)$. Conforme demonstrado em WEELER \& STERNBERG (1999), se essa função tem sua segunda derivada em relação ao tempo contínua, ao longo de um intervalo aberto em torno do tempo " $t$ ", o comportamento limite dos campos fundamentais, quando $q \rightarrow \mathbf{s}$, são representados esquematicamente por:

$$
\begin{array}{ll}
\lim _{q \rightarrow s} u_{k i}^{*}(q, \tau, s / f)=0\left(r^{-1}\right) & \forall \tau \in[t-\varepsilon, t+\varepsilon] \\
\lim _{q \rightarrow s} u_{k i}^{*}(q, \tau, s / f)=0\left(r^{-2}\right) & \forall \tau \in[t-\varepsilon, t+\varepsilon]
\end{array}
$$

Também foi demonstrado que: 


$$
\lim _{r \rightarrow 0} \int_{S_{\alpha}} p_{k i}^{*}(q, \tau ; s / f) d \Omega(q)=f(\tau) \quad \forall \tau \in[t-\varepsilon, t+\varepsilon]
$$

onde $S_{\Omega}$ é uma superfície esférica de raio " $r$ ".

Levando-se em conta essas condições, a função $f(\tau)$ adotada nesse trabalho é:

$$
f(\tau)=(H(\tau)-H(\tau+\Delta \tau)) / \Delta \tau
$$

onde $\mathrm{H}(\tau)$ é a função Heaviside. Essa função $f(\tau)$, escrita em IV.16, representa um impluso unitário quando integrado ao longo de um intervalo de tempo $\Delta \tau$. Substituindo-se IV.16 em IV.10 obtém-se a carga concentrada na direção $k$, agindo em um ponto qualquer $s$ do domínio infinito, expressa por:

$$
b_{k i}^{*}(q, \tau, s, 0)=(H(\tau)-H(\tau+\Delta \tau)) \delta(s-q) \delta_{k i} / \Delta \tau
$$

Uma vez assumida a função $f(\tau)$, obtém-se a solução fundamental substituindo-se $f(\tau)$ em IV.11. Desta forma tém-se que:

$$
u_{k i}^{*}(q, \tau ; s, 0)=\left(A_{k i}^{*}(q, \tau, s, 0)-A_{k i}^{*}(q, \tau+\Delta t, s, 0)\right) / \Delta t
$$

onde:

$$
\begin{aligned}
A_{k i}^{*}(q, \tau ; s, 0)= & \frac{1}{4 \pi \rho \Delta t}\left\{\left(\frac{3 r_{i} r_{k}}{r^{3}}-\frac{\delta_{k i}}{r}\right) \frac{1}{2 r^{2}}\left[\left(\tau^{2}-\frac{r^{2}}{C_{1}^{2}}\right) H\left(\tau-\frac{r}{C_{1}}\right)-\left(\tau^{2}-\frac{r^{2}}{C_{2}^{2}}\right) \cdot H\left(\tau-\frac{r}{C_{2}}\right)\right]\right. \\
& \left.+\frac{r_{i} r_{k}}{r^{3}}\left[\frac{1}{C_{1}^{2}} H\left(\tau-\frac{r}{C_{1}}\right)+\frac{1}{C_{2}^{2}} H\left(\tau-\frac{r}{C_{2}}\right)\right]+\frac{\delta_{k j}}{r C_{2}^{2}} H\left(\tau-\frac{r}{C_{2}}\right)\right\}
\end{aligned}
$$




\subsection{2 - Equações integrais}

O teorema da reciprocidade de Graffi para problemas dinâmicos pode ser escrito para dois estados elastodinâmicos, aqui representados pelos campos de deslocamentos u e u', como segue [MANSUR (1985)]:

$$
\begin{aligned}
& \int_{0}^{t} \int_{\Omega}\left[u_{i}(q, \tau) b_{i}(q, t-\tau)-u_{i}^{\prime}(q, t-\tau) b_{i}(q, \tau)\right] d \Omega d \tau+\rho \int_{\Omega}\left\{\dot{u}_{i}(q, t) u_{i}^{\prime}(q, 0)+u_{i}(q, t) \dot{u}_{i}(q, 0)\right\} d \Omega \\
& =\int_{0}^{1} \int_{\Gamma}\left\{u_{i}(Q, t-\tau) p_{i}(Q, \tau)-u_{i}(Q, \tau) p_{i}^{\prime}(Q, t-\tau)\right\} d \Gamma d \tau+\rho \int_{\Omega}\left\{\dot{u}_{i}(q, t) u_{i}(q, 0)+u_{i}^{\prime}(q, t) \dot{u}(q, 0)\right\} d \Omega
\end{aligned}
$$

onde $\Omega$ é uma região fechado ou aberta, e $\Gamma$ o seu contorno.

Sem perda de generalização, apenas o caso do estado elastodinâmico de "quiescent past" será estudado. Assim a equação IV.20 fica escrita como:

$$
\begin{aligned}
& \int_{0} \int_{\Omega}\left[u_{i}(q, \tau) b_{i}^{\prime}(q, t-\tau)-u_{i}^{\prime}(q, t-\tau) b_{i}(q, \tau)\right] d \Omega d \tau \\
& =\int_{0}^{\tau}\left\{\left\{u_{i}^{\prime}(Q, t-\tau) p_{i}(Q, \tau)-u_{i}(Q, \tau) p_{i}(Q, t-\tau)\right\} d \Gamma d \tau\right.
\end{aligned}
$$

Substituindo a solução geral de Stokes, equações IV.11 e IV.12, na equação anterior tém-se que:

$$
\begin{aligned}
& \int_{0}^{t} \int_{\Omega} u_{i}(q, \tau) f(t-\tau) \delta(q-s) \delta_{k i} d \Omega d \tau=\int_{0}^{t} \int_{\Gamma} u_{k i}^{*}(Q, t-\tau ; s / f) p_{i}(Q, \tau) d \Gamma d \tau \\
& -\int_{0}^{t} \int_{\Gamma} u_{i}(Q, \tau) p_{k i}^{*}(Q, t-\tau ; s / f) d \Gamma d \tau+\int_{0}^{t} \int_{\Omega} u_{k i}^{*}(q, t-\tau ; s / f) b_{i}(q, \tau) d \Omega d \tau
\end{aligned}
$$

Levando-se em conta as propriedades IV.13 a IV.15 obtém-se: 
$C_{k i}(Q, s) \int_{0}^{t} u_{i}(s, \tau) f(t-\tau) d \tau=\int_{0}^{t} \int_{\Gamma} u_{i}^{*}(Q, t-\tau ; s / f) p_{i}(Q, \tau) d \Gamma d \tau$

$-\int_{0}^{t} \int_{\mathrm{r}} u_{i}(Q, \tau) p_{k i}^{\cdot}(Q, t-\tau ; s / f) d \Gamma d \tau+\int_{0}^{t} \int_{\Omega} u_{k i}^{*}(q, t-\tau ; s / f) b_{i}(q, \tau) d \Omega d \tau$

onde $C_{k i}(Q, s)$ é o clássico termo livre da representação integral do deslocamento que pode ser facilmente calculado, como em HARTMANN (1980). O símbolo $f$ indica que a integral é fortemente singular e deve ser calculada no sentido do valor principal de Cauchy.

Para se escrever a representação integral do deslocamento basta substituir a função $f(\tau)$ da equação IV.23 pela dada em IV.16, da mesma forma feita para obter a solução fundamental IV.18. Uma vez feita essa substituição tém-se:

$$
\int_{\delta}^{[}[H(t-\tau)-H(t-(\tau+\Delta t))] u_{i}(q, \tau) d \tau=\int_{t-\Delta t} u_{i}(q, \tau) d \tau
$$

ou seja:

$$
\begin{aligned}
& C_{k i}(Q, s) \int_{t-\Delta t}^{t} \frac{u_{i}(s, \tau)}{\Delta t} d \tau=\int_{0}^{t} \int_{\Omega} u_{k i}^{*}(Q, t ; s, \tau) p_{i}(Q, \tau) d \Gamma d \tau \\
& -\int_{0}^{t} \int_{\Gamma} u_{i}(Q, \tau) p_{k i}^{*}(Q, t ; s, \tau) d \Gamma d \tau+\int_{0}^{1} \int_{\Omega} u_{k i}^{*}(q, t ; s, \tau) b_{i}(q, \tau) d \Omega d \tau
\end{aligned}
$$

Na utilização das equações IV.24 e IV.25 deve-se levar em conta as seguintes propriedades do estado de Stokes com respeito aos valores fundamentais

$$
u_{k i}^{*}(Q, t-\tau ; s, 0)=u_{k i}^{*}(Q, t ; s, \tau)
$$




$$
p_{k i}^{*}(Q, t-\tau ; s, 0)=p_{k i}^{*}(Q, t ; s, \tau)
$$

\subsection{3 - Aspectos numéricos}

Para a aplicação do Método dos Elementos de Contorno a equação integral IV.25 deve ser tratada numericamente, adotando-se apropriadas discretizações de espaço e tempo. Devido às características da solução fundamental proposta, nenhuma restrição é feita com respeito à discretização a ser empregada. Para o caso particular da discretização no tempo, elementos constantes foram adotados por simplicidade, bem como pela acuidade dos resultados numéricos verificados em todas as análises feitas. Reescrevendo-se a equação IV.25, assumindo as discretizações espaciais e temporais, tém-se que:

$$
\begin{aligned}
& C_{k i}(s) U_{i}(s, t)^{N_{t}}=\int_{\mathcal{H}_{(j)}} \int_{\tau}^{(\theta)} u_{k i}^{(\theta)}(Q, t ; s, \tau) \phi_{\alpha}^{j}(Q) \psi^{\theta}(\tau) d \tau d \Gamma(Q) P_{i \theta}^{\alpha j} \\
& -\int_{\{(j)} \int_{i(\theta-1)}^{(\theta)} p_{k i}^{*}(Q, t ; s, \tau) \phi_{\alpha}^{j}(Q) \psi^{\theta}(\tau) d \tau d \Gamma(Q) U_{i \theta}^{a j}
\end{aligned}
$$

onde $\theta$ varia de 1 até o n-ésimo passo de integração $N_{t}$, e a regra do somatório é aplicada.

Como é usual no método, para se encontrar a representação algébrica final, os deslocamentos e esforços são aproximados por:

$$
U_{i \theta}\left(Q^{j}, t\right)=\phi_{\alpha}^{(j)} \psi^{(\theta)} U_{i(\theta)}^{\alpha(j)}
$$




$$
P_{i \theta}\left(Q^{j}, t\right)=\phi_{\alpha}^{(j)} \psi^{(\theta)} P_{i(\theta)}^{\alpha(j)}
$$

onde $j$ significa representação espacial, $\phi_{k}$ é a função de aproximação no espaço, enquanto $\psi$ dá a aproximação ao longo do tempo.

Os elementos triangulares planos e quadrangulares planos, com três e quatro nós cada, respectivamente, foram adotados para a discretização no espaço. Os elementos singulares foram computados com um algoritimo especial onde a quadratura de Kutt foi empregada para uma pequena região em torno do ponto de carregamento e a quadratura de Gauss foi utilizada para o restante do elemento, junto com uma apropriada subdivisão dos elementos. Para as integrais não singulares a quadratura de Gauss foi adotada com a subdivisão de elementos.

Agora, para integrar a parte dependente do tempo da equação IV.18 assume-se $\psi=1$ para cada passo de tempo, dando assim:

$$
\int_{t_{\theta-1}}^{\theta_{0}} \frac{1}{\Delta t}\left[(t-\tau)^{2}-\frac{r^{2}}{C_{\gamma}^{2}}\right] H\left(t-\tau-\frac{r}{C_{\gamma}}\right) d \Gamma
$$




$$
\begin{aligned}
& =\left\{\begin{array}{c}
\frac{1}{\Delta t}\left\{\left(t^{2}-\frac{r^{2}}{C_{\gamma}^{2}}\right) \Delta t-t\left(t_{\theta}^{2}-t_{\theta-1}^{2}\right)+\left(t_{\theta}^{3}-t_{\theta-1}^{3}\right) / 3\right\} \\
\frac{1}{\Delta t}\left\{\left(t^{2}-\frac{r^{2}}{C_{\gamma}^{2}}\right)\left(T A-t_{\theta-1}\right)-t\left(T A^{2}-t_{\theta-1}^{2}\right)+\left(T A^{3}-t_{\theta-1}^{3}\right) / 3\right\} \\
0
\end{array}\right\} \\
& \text { if } t-t_{\theta} \geq \frac{r}{C_{\gamma}} \\
& \text { if } t-t_{\theta-1} \geq \frac{r}{C_{\gamma}} \geq t-t_{\theta} \\
& \text { if } \frac{r}{C_{\gamma}} \geq t-t_{\theta-1}
\end{aligned}
$$

$$
\begin{aligned}
& \int_{t_{\theta-1}}^{f_{\theta}} \frac{1}{\Delta t} H\left(t-\tau-\frac{r}{C_{\gamma}}\right) d \tau \\
& =\left\{\begin{array}{clc}
\frac{\left(T A-t_{\theta-1}\right)}{\Delta t} & \text { if } & t-t_{\theta} \geq \frac{r}{C_{\gamma}} \\
0 & \text { if } & t-t_{\theta} \geq \frac{r}{C_{\gamma}} \geq t-t_{\theta}
\end{array}\right. \\
&
\end{aligned}
$$

$$
\int_{t_{\theta-1}}^{f_{\theta}} \frac{1}{\Delta t} \delta\left(t-\tau-\frac{r}{C_{\gamma}}\right) d \tau
$$


$=\left\{\begin{array}{llc}0 & \text { if } & t-t_{\theta}>\frac{r}{C_{\gamma}} \text { or } \frac{r}{C_{\gamma}}>t-t_{\theta-1} \\ 0 & \text { if } & t-t_{\theta-1} \geq \frac{r}{C_{\gamma}} \geq t-t_{\theta}\end{array}\right.$

onde

$$
T A=t-r / C_{\gamma}
$$

Subtraindo dos valores obtidos pelas equações IV.29, IV.30 e IV.31 os correspondentes computados no próximo passo de tempo, $\left[t_{\theta}, t_{\theta+1}\right]$, as constantes no tempo da solução fundamental IV.18 são obtidas. Uma alternativa para fazer a convolução no tempo da solução fundamental pode ser obtida direto da sua expressão explícita.

$\mathrm{Na}$ formulação do MEC, a representação integral do deslocamento é transformada em uma equação algébrica após efetuadas todas as integrações no espaço e no tempo sobre os elementos. Uma vez obtido o sistema algébrico, pode-se representar o sistema linear de equaçōes da seguinte forma:

$$
\underset{\sim}{H}{ }_{\sim}^{\theta} U^{\theta}=G_{\sim}^{\theta} P^{\theta}
$$

onde $\theta=1,2, \ldots, N_{t}$. 


\section{3 - Acoplamento placa-solo}

Uma vez implementadas as formulaçōes para análise de cada estrutura separadamente, pode-se passar à análise da associação entre as duas. Estas formulações estão escritas em II.79 e IV.11, para o caso elastostático, e em III.14, III.15 e IV.19, para o caso elastodinâmico, e podem ser escritas, genericamente, como sendo:

$$
\underset{\sim}{H} \underset{\sim}{U}=\underset{\sim}{G} \underset{\sim}{P}+\underset{\sim}{F}
$$

Para proceder o acoplamento de diferentes estruturas usando a técnica de subregiões basta aplicar a equação IV.34 separadamente a cada região e, em seguida, interligá-las usando condições de compatibilidade de equilíbrio e deslocamentos. Seguindo o que foi dito, tendo-se um domínio formado por várias subregiões, escreve-se a equação IV.34 para todas as subregiőes, da seguinte forma:

$$
{\underset{\sim}{H}}^{(i)} \underline{\sim}^{(i)}={\underset{\sim}{G}}^{(i)}{\underset{\sim}{(i)}}^{\left(F^{(i)}\right.}
$$

onde $i$ indica a subregião correspondente à equação.

Seja uma interface $\Gamma_{i j}$ entre duas subregiōes $i$ e $j$, as condições de equilíbrio de compatibilidade de deslocamentos são dadas por:

$$
\begin{aligned}
& {\underset{\sim}{U}}^{(i j)}=\underline{\sim}^{(j i)} \\
& P^{(i j)}+P^{(j i)}=0
\end{aligned}
$$


onde o primeiro indice indica a região à qual correspondem os deslocamentos e esforços, enquanto o segundo índice indica a subregião adjacente.

Como pode-se notar, os dois vetores $U$ e $P$ se referem apenas aos valores de contorno que estão contidos na interface entre as subregiões. Podem existir, contudo, valores externos à interface, e a forma completa dos vetores pode ser escrita como sendo formada por duas parcelas, ou seja:

$$
\begin{aligned}
& {\underset{\sim}{U}}^{(i)}=\left[\begin{array}{c}
U^{(i e)} \\
\tilde{U}^{(i j)}
\end{array}\right] \\
& \stackrel{\sim}{P}^{(i)}=\left[\begin{array}{c}
\mathcal{P}^{(i e)} \\
\tilde{P}^{(i j)}
\end{array}\right]
\end{aligned}
$$

onde o indice ie corresponde aos valores externos à interface.

Com esta forma de separar em duas partes, a equação IV.35 pode ser reescrita para uma subregião i como:

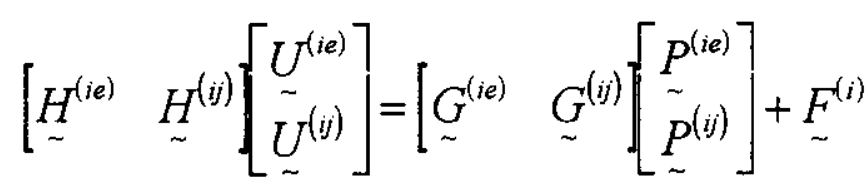

O mesmo pode ser feito para a subregião j,

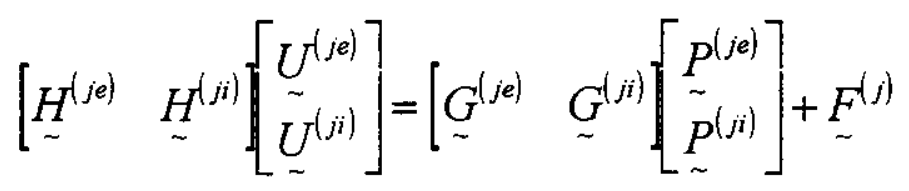


Escritas as equações para as diferentes subregiões, e levando-se em conta as condições IV.36, pode-se acoplar o sistema em uma única expressão matricial:

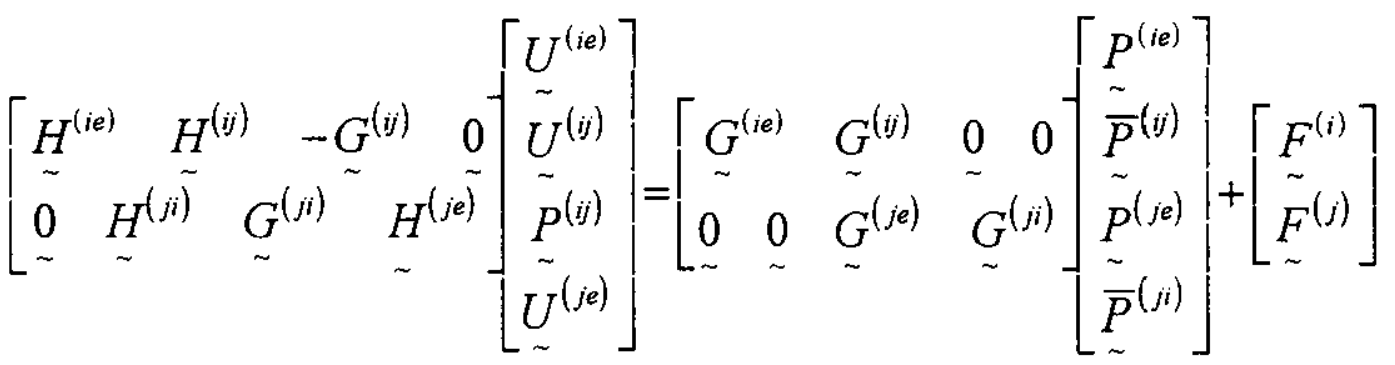

onde a barra indica carregamento prescrito na interface.

Resolvendo-se IV.41 determinam-se os valores incógnitos da interação placa-solo.

\section{4 - Exemplos numéricos}

Para mostrar o comportamento da formulação apresenta-se, nesse ítem, alguns exemplos numéricos. O primeiro exemplo apresentado se trata de um caso elastostático encontrado em PAIVA (1994). A escolha de se iniciar por esse exemplo se justifica pela necessidade de melhor ilustrar a acuidade da formulação bem como de permitir as futuras comparações com a formulação em elementos finitos apresentada por CODA (1993). No caso da formulação via elementos finitos foram utulizados 96 elementos LCCT9, enquanto que os detalhes do exemplo e da discretização empregadas nesse trabalho são apresentados a seguir: 
exemplo 10 (estático): figura 19

$$
\begin{aligned}
& h=0.1 \mathrm{~m} \\
& E_{P}=9.78 \times 10^{10} \mathrm{KN} / \mathrm{m}^{3} \\
& E_{S}=0.26 \times 10^{6} \mathrm{KN} / \mathrm{m}^{3} \\
& v_{S}=v_{P}=0.3
\end{aligned}
$$
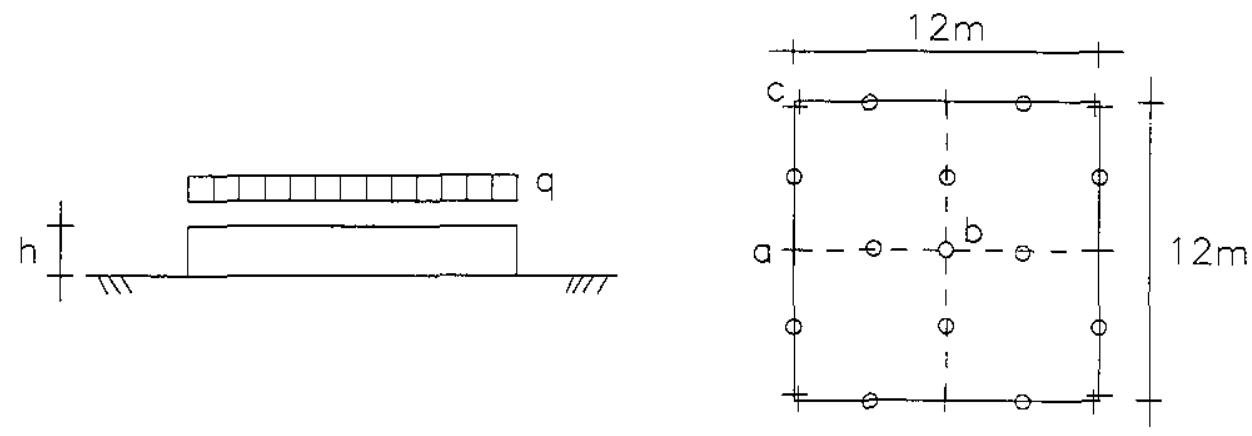

FIGURA 19

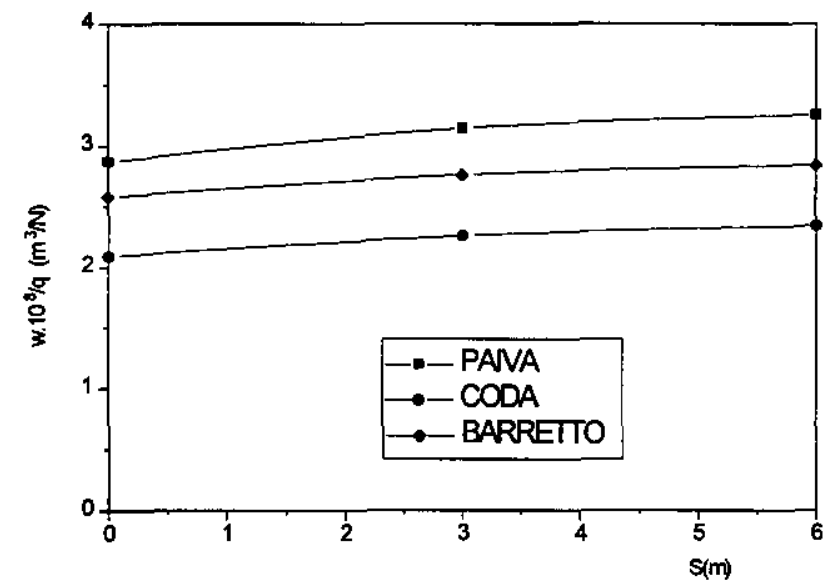

GRÁFICO 19 - deslocamento vertical ao longo de a-b 


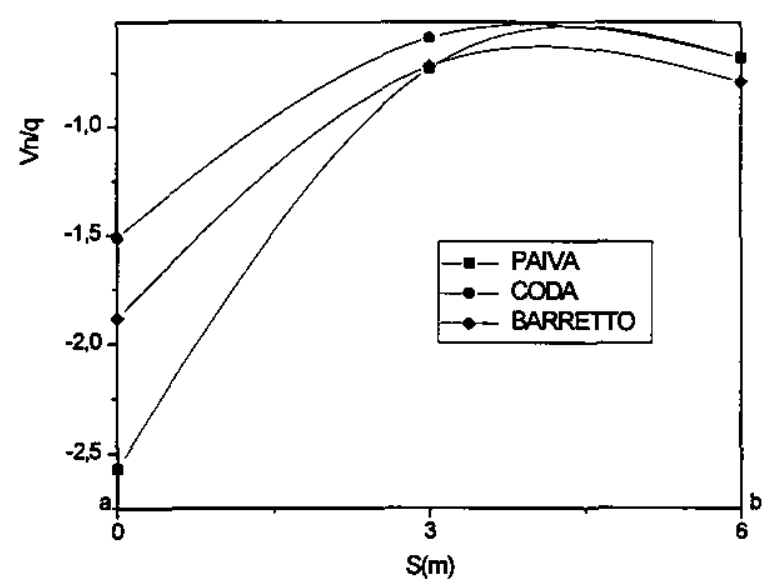

GRÁFICO 20 - reação normal ao longo de a-c

De agora em diante todos os exemplos, estáticos ou dinâmicos, serão apresentados com os resultados comparados com os obtidos pela formulação via elementos finitos. O primeiro exemplo a ser apresentado a seguir se trata de mais um exemplo elastostático, submetido a um carregamento transversal uniformemente distribuído, onde se analisa uma placa com espessura de 50 $\mathrm{cm}$, objetivando ilustrar os problemas que as concentrações de tensão nas bordas do contato podem causar numa análise elastodinâmica. A partir de então, três exemplos dinâmicos e um estático são apresentados, sendo o primeiro dinâmico (ex. 12) com as mesmas características do exemplo estático (ex.11). Em seguida é mostrado o comportamento elastodinâmico de uma placa submetida a uma carga concentrada no seu centro e, finalmente, apresenta-se o caso de uma ligação placa-solo em que ambos os módulos de elasticidade, da placa e do solo, são iguais. Nesse exemplo apenas os resultados obtidos com a formulação desenvolvida nesse trabalho é apresentada e, devido à ausência de concentração de tensão, pode-se notar que se consegue obter uma resposta da estabilizada ao longo do tempo da análise, quando da análise 
elastodinâmica. Em todos os exemplos dinâmicos a carga é considerada agindo constante ao longo do tempo de análise.

Os detalhes de geometria, constantes empregadas e carregamento estão descritos juntos com os exemplos.

exemplo 11 (estático): figura 19

$$
\begin{aligned}
& h=0.5 \mathrm{~m} \\
& E_{P}=7.8 \times 10^{10} \mathrm{Kg} / \mathrm{m} / \mathrm{s}^{3} \\
& E_{S}=7.8 \times 10^{8} \mathrm{Kg} / \mathrm{m} / \mathrm{s}^{3} \\
& v_{S}=v_{P}=0.3
\end{aligned}
$$
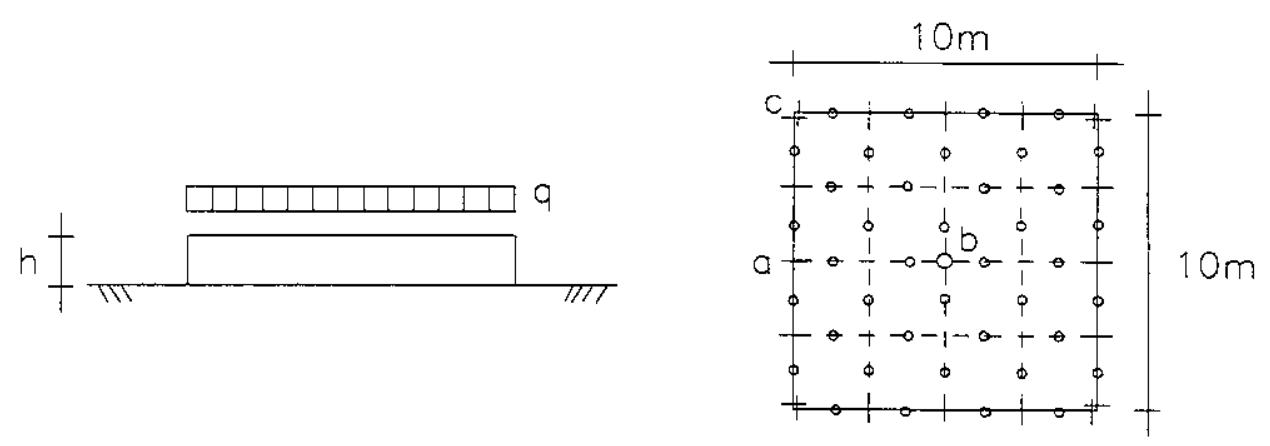

FIGURA 19

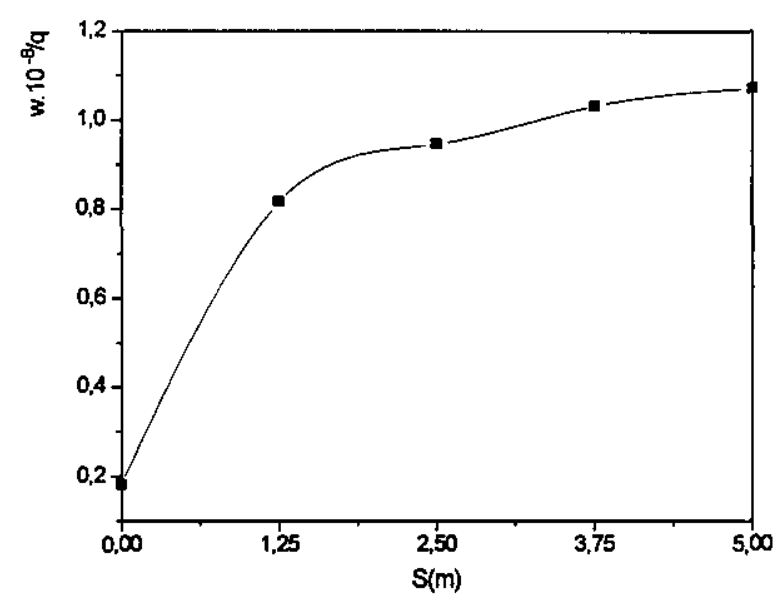

GRÁFICO 21 - deslocamento vertical ao longo de a-b 


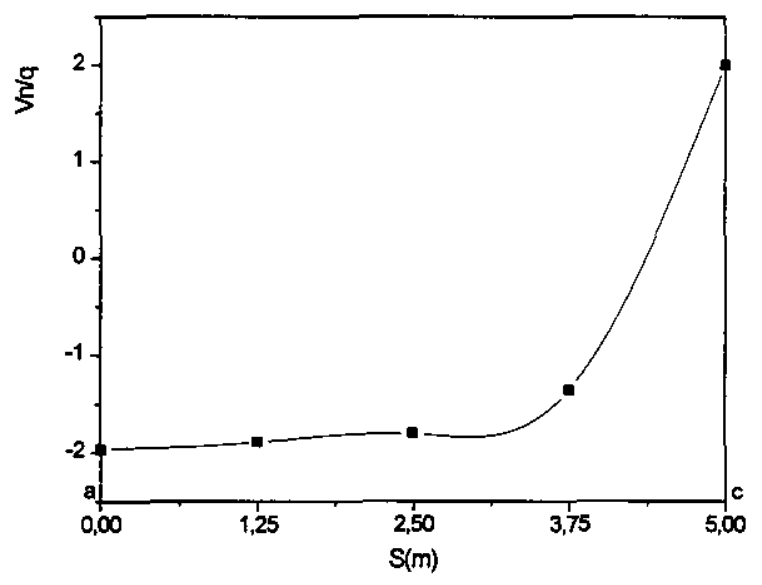

GRÁFICO 22 - reação normal ao longo de a-c

exemplo 12 (dinâmico): figura 19

$$
\begin{aligned}
& h=0.5 \mathrm{~m} \\
& E_{P}=7.8 \times 10^{10} \mathrm{Kg} / \mathrm{m}^{3} \mathrm{~s}^{3} \\
& E_{S}=7.8 \times 10^{8} \mathrm{Kg} / \mathrm{m} / \mathrm{s}^{3} \\
& \rho_{P}=5000 \mathrm{Kg} / \mathrm{m}^{3} \\
& \rho_{S}=3600 \mathrm{Kg} / \mathrm{m}^{3} \\
& v_{S}=v_{P}=0.3
\end{aligned}
$$




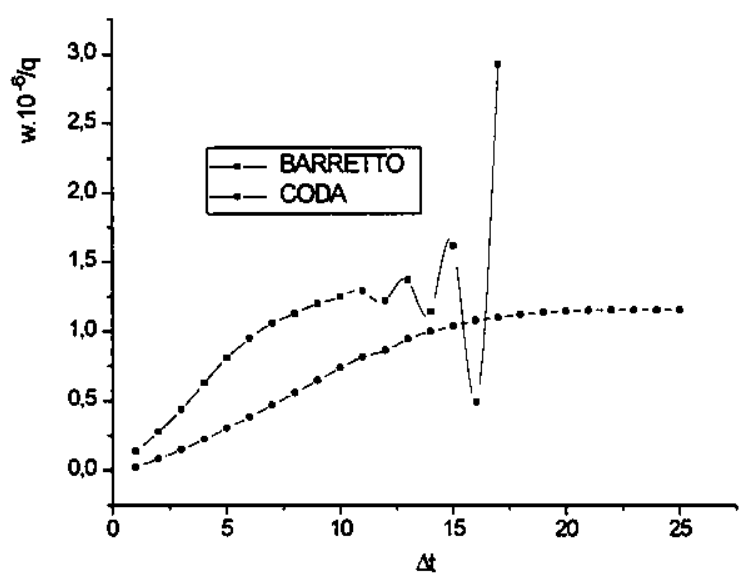

GRÁFICO 23 - deslocamento vertical ao longo de a-b

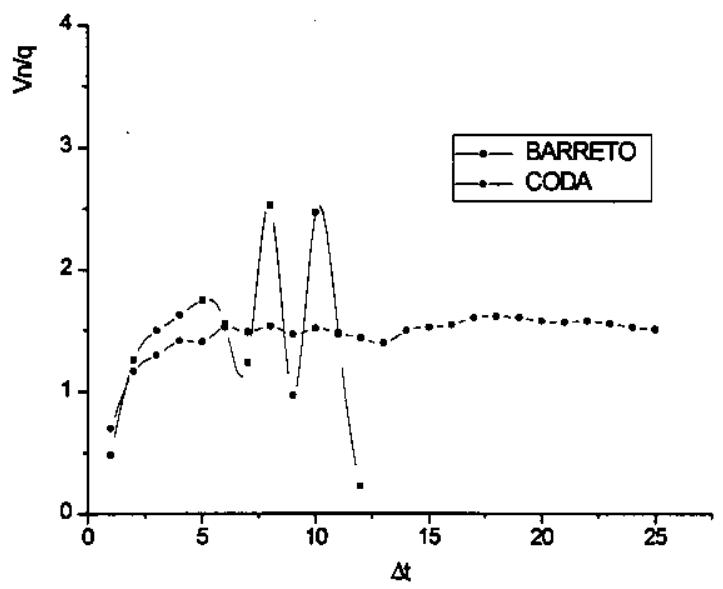

GRAFICO 24 - reação normal ao longo de a-c 
exemplo 13 (dinâmico): figura 20

$\mathrm{h}=0.5 \mathrm{~m}$

$E_{p}=7.8 \times 10^{10} \mathrm{Kg} / \mathrm{m} / \mathrm{s}^{3}$

$E_{S}=7.8 \times 10^{8} \mathrm{Kg} / \mathrm{m} / \mathrm{s}^{3}$

$\rho_{\mathrm{P}}=5000 \mathrm{Kg} / \mathrm{m}^{3}$

$\rho_{\mathrm{S}}=3600 \mathrm{Kg} / \mathrm{m}^{3}$

$v_{S}=v_{P}=0.3$
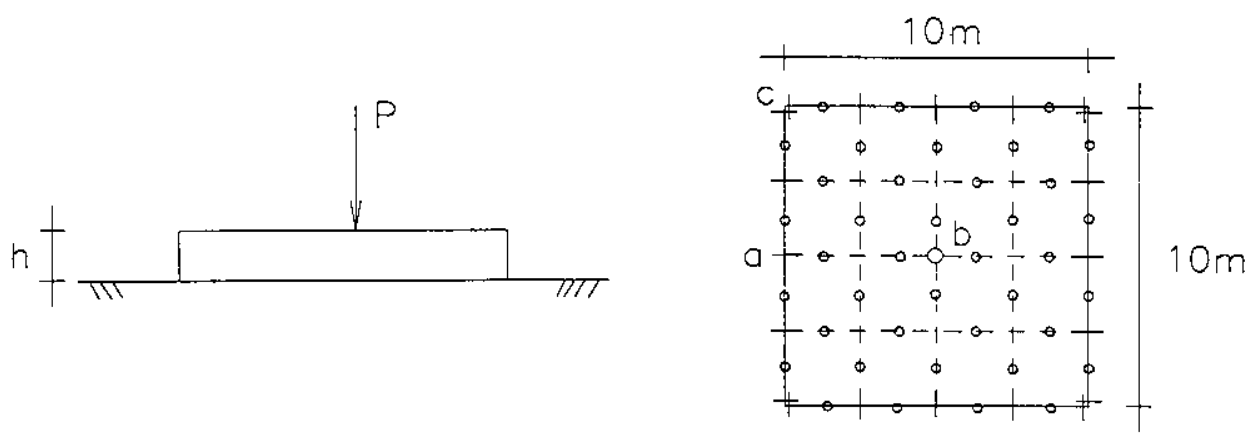

FIGURA 20

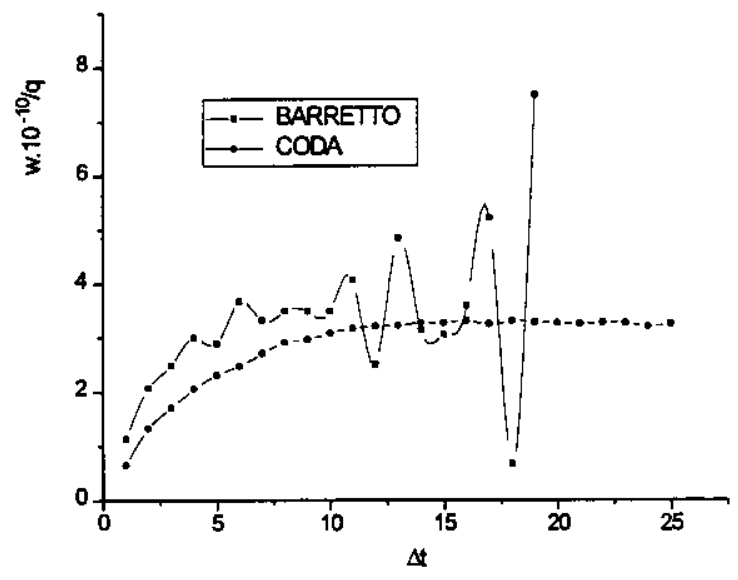

GRÁFICO 25 - deslocamento vertical ao longo de a-b 


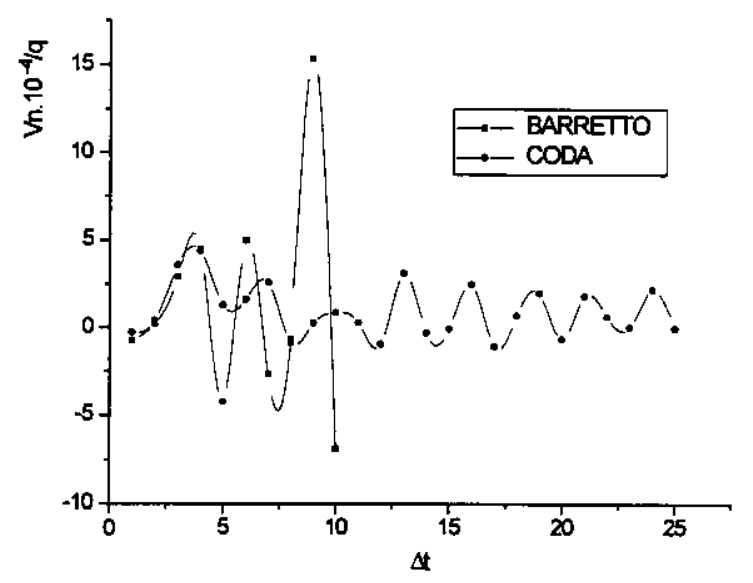

GRÁFICO 26 - reação normal ao longo de a-c

exemplo 14 (estático): figura 19

$$
\begin{aligned}
& h=0.5 \mathrm{~m} \\
& E_{P}=E_{S}=10^{7} \mathrm{Kg} / \mathrm{m} / \mathrm{s}^{3} \\
& v_{S}=v_{P}=0.3
\end{aligned}
$$




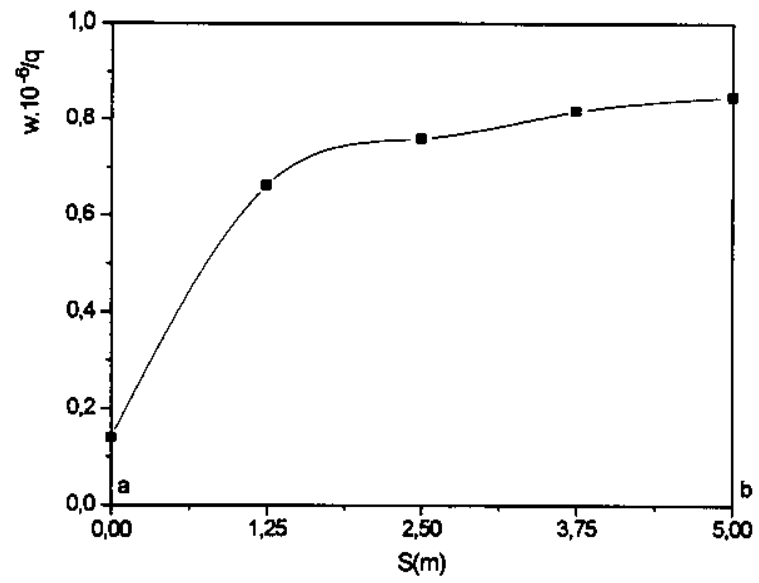

GRÁFICO 27 - deslocamento vertical ao longo de a-b

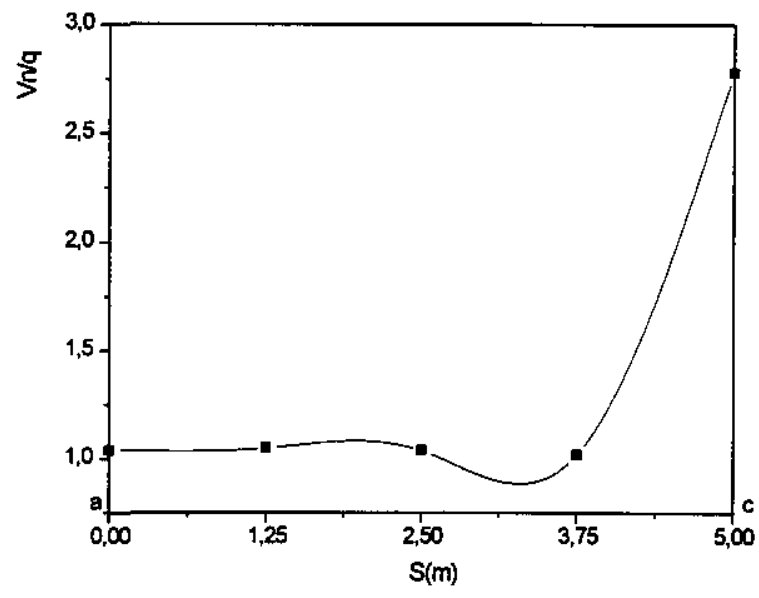

GRÁFICO 28 - reação normal ao longo de a-c 
exemplo 15 (dinâmico): figura 19

$$
\begin{aligned}
& h=0.5 \mathrm{~m} \\
& E_{P}=E_{S}=10^{7} \mathrm{Kg} / \mathrm{m} / \mathrm{s}^{3} \\
& \rho_{P}=0.0002588 \mathrm{Kg} / \mathrm{m}^{3} \\
& v_{S}=v_{P}=0.3
\end{aligned}
$$

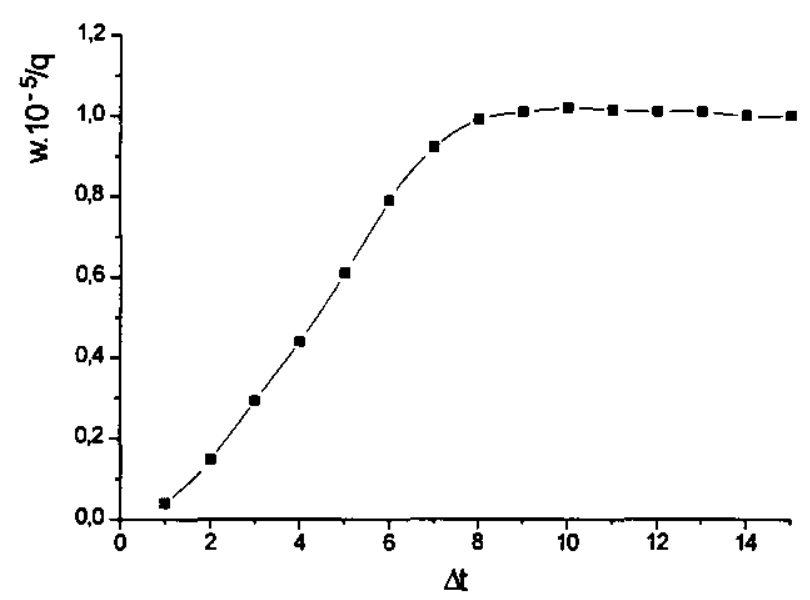

GRÁFICO 29 - deslocamento vertical ao longo de a-b

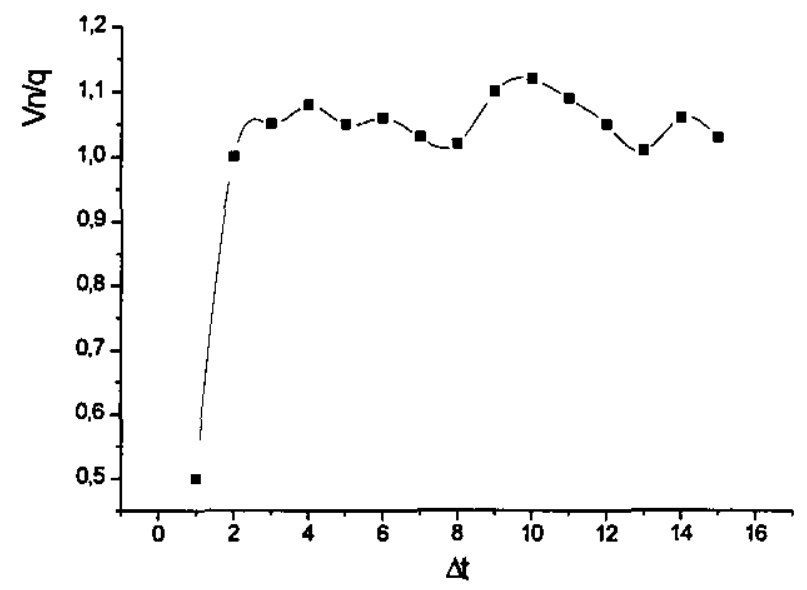

GRÁFICO 30 - reação normal ao longo de a-c 
Nesse último exemplo elastodinâmico (ex.15) foram empregadas constantes diferentes dos outros exemplos. Isso se explica pela necessidade de se dimuinuir o $\Delta t$ da análise para se obter a estabailidade. Na ligação entre o solo e a placa, a matriz resultante acaba sempre apresentando problemas de condicionamento, o que impede que se possa manipular de um modo mais amplo o passo de tempo $(\Delta t)$ utilizado na análise. Com o emprego das constantes do exemplo 15 foi possivel reduzir esse passo de tempo até o necessário para se obter a resposta apresentada. Nos outros exemplos dinâmicos, uma outra forma de acoplar o solo com a placa (sub-estrutura, por exemplo) ou mesmo o emprego de uma resolução iterativa do sistema final podem contribuir para suavizar o comportamento ilustrados nas figuras 23 a 26. 


\section{CAPÍTULO $V$}

\section{ANÁLISE DO COMPORTAMENTO ELASTODINÂMICO DE PLACAS UTILIZANDO-SE SOLUÇÃO FUNDAMENTAL TRANSIENTE, NO DOMÍNIO DO TEMPO}

\section{1 - Introdução}

Neste capítulo será apresentada a formulação para análise elastodinâmica transiente de placas, proposta por BÉZINE (1982).

Inicialmente apresenta-se o desenvolvimento da equação integral para a análise elastodinâmica a partir da Identidade de Green e das propriedades do produto de convolução.

Em seguida demonstra-se a obtenção da solução fundamental apresentada por Bézine. A sua obtenção parte do trabalho de SNEDDON (1944), que obteve uma solução analítica, utilizando-se da Transformada de Hankel, para o deslocamento central de uma placa circular, de raio infinito, com carga concentrada apticada no centro $(r=0)$. 
No final do capítulo a formulação aqui desenvolvida será aplicada numa placa circular, simplesmente apoiada, com um carregamento concentrado no centro, onde se pretende ilustrar o comportamento da solução.

\section{2 - Equação integral transiente da placa}

Sejam $\boldsymbol{w}$ e $\boldsymbol{w}^{*}$, duas funções referidas às variáveis de espaço $x=\left(x_{1}, x_{2}\right)$ e de tempo $t$, que satisfazem as equações de movimento da placa, ou seja:

$$
\begin{aligned}
& b^{2} \Delta \Delta w+\frac{\partial^{2} w}{\partial t}=\frac{f(x, t)}{2 \rho h} \\
& b^{2} \Delta \Delta w^{*}+\frac{\partial^{2} w^{*}}{\partial t}=\frac{\delta(x) H(t)}{2 \rho h}
\end{aligned}
$$

onde $\delta_{(x)}$ é a função Delta de Dirac, cujas propriedades estão escritas em II.23, $\boldsymbol{H}_{(t)}$ é a função Heaviside que é definida por:

$$
\begin{array}{ll}
H(t)=1 \quad \text { para } \mathrm{t} \in \mathrm{T}^{+} \\
H(t)=0 \quad \text { para } \mathrm{t}<0 \\
\text { e } \quad b^{2}=\frac{D}{2 \rho h}
\end{array}
$$

sendo $h$ a espessura da placa e $\rho$ a densidade do material. 
O ponto inicial para a obtenção da equação integral é o teorema da reciprocidade, obtido a partir da segunda Identidade de Green ( BERGMAN (1953) ), que pode escrita por,

$$
\left.\int_{\Omega}\left(w^{*} \Delta \Delta w-\Delta w^{*} \Delta w\right) d \Omega=\int w_{\Gamma}^{*} \frac{\partial \Delta w}{\partial n}-\frac{\partial w^{*}}{\partial n} \Delta w\right) d \Gamma
$$

Pode-se também facilmente demonstrar que:

$$
\int_{\Omega}\left(w^{*} \Delta \Delta w-\Delta w^{*} \Delta w+p\left(w^{*}, w\right)\right) d \Omega=\frac{1}{D} \int_{\Gamma}\left(\frac{\partial w^{*}}{\partial n} M_{n}(w)+\frac{\partial w^{*}}{\partial s} M_{n t}(w)+D w^{*} \frac{\partial \Delta w}{\partial n}\right) d \Gamma
$$

onde:

$$
p\left(w^{*}, w\right)=\int_{\Omega}(1-v)\left(\frac{\partial^{2} w^{*}}{\partial x^{2}} \frac{\partial^{2} w}{\partial y^{2}}-2 \frac{\partial^{2} w^{*}}{\partial x \partial y} \frac{\partial^{2} w}{\partial x \partial y}+\frac{\partial^{2} w^{*}}{\partial y^{2}} \frac{\partial^{2} w}{\partial x^{2}}\right) d \Omega
$$

sendo as expressões de $\boldsymbol{M}_{n}$ e $\boldsymbol{M}_{\boldsymbol{n}}$ obtidas a partir das expressões II.10, II.11, II.4, 11.5 e 11.5 .

A segunda parcela da integral de contorno V.6 pode ser reescrita como sendo:

$$
\frac{\partial w^{*}}{\partial s} M_{n t}(w)=\frac{\partial}{\partial s}\left[w^{*} M_{n t}(w)\right]-w^{*} \frac{\partial M_{n t}(w)}{\partial s}
$$


Conforme já foi explicado no capítulo II,

$$
\int_{\Gamma} \frac{\partial}{\partial s}\left[w^{*} M_{n t}(w)\right] d \Gamma=0
$$

para os casos em que a integração é feita numa placa de contorno fechado, em que não haja cantos. Para o caso de haver cantos, tém-se que:

$$
\int_{\Gamma}^{\partial} \frac{\partial}{\partial s}\left[w^{*} M_{n t}(w)\right] d \Gamma=-\sum_{i=1}^{N_{c}}\left[w^{*} M_{n t}(w)\right]_{i}
$$

onde $\boldsymbol{N}_{c}$ indica o número de cantos da placa.

Com esta transformação pode- se reescrever V.5 da seguinte forma:

$$
\begin{aligned}
\int_{\Omega}\left(w^{*} \Delta \Delta w-\Delta w^{*} \Delta w+p\left(w^{*}, w\right)\right) d \Omega= & \frac{1}{D} \int_{\Gamma}\left(\frac{\partial w^{*}}{\partial n} M_{n}(w)-w^{*} \frac{\partial M_{n t}(w)}{\partial s}+D w^{*} \frac{\partial \Delta w}{\partial n}\right) d \Gamma \\
& -\frac{1}{D} \sum_{i=1}^{N c}\left[w^{*} M_{n t}(w)\right]
\end{aligned}
$$

Fazendo-se o mesmo procedimento para

$$
\int_{\Omega}\left(w \Delta \Delta w^{*}-\Delta w \Delta w^{*}+p\left(w^{*}, w\right)\right) d \Omega
$$

e somando-se os dois resultados tém-se que: 


$$
\begin{aligned}
& \int_{\Omega}\left(w^{*} \Delta \Delta w-w \Delta \Delta w^{*}\right) d \Omega=-\frac{1}{D} \int_{\Gamma}\left[V_{n}(w) w^{*}-M_{n}(w) \frac{\partial w^{*}}{\partial n}+\frac{\partial w}{\partial n} M_{n}\left(w^{*}\right)\right. \\
& \left.-w V_{n}\left(w^{*}\right)\right] d \Gamma+\frac{1}{D} \sum_{i+1}^{N c}\left\{\left[w M_{n t}\left(w^{*}\right)-w^{*} M_{n t}(w)\right]_{i}\right\}
\end{aligned}
$$

Levando-se em conta as equações V.1 e V.2, e as seguintes propriedades do produto de convolução (o sinal indicando produto de convolução entre as funções variáveis no tempo será, por simplicidade, omitido nas equações):

$$
\begin{aligned}
& \frac{\partial^{2} w^{*}}{\partial^{2}} w=\frac{\partial^{2}}{\partial^{2}}\left(w^{*} w\right)-\left(\frac{\partial w^{*}}{\partial t}\right)_{0} w-w_{0} \frac{\partial w^{*}}{\partial t} \\
& \frac{\partial^{2} w}{\partial^{2}} w^{*}=\frac{\partial^{2}}{\partial^{2}}\left(w w^{*}\right)-\left(\frac{\partial w}{\partial t}\right)_{0} w^{*}-w_{0}^{*} \frac{\partial w}{\partial t}
\end{aligned}
$$

onde:

$$
\begin{array}{ccc}
w_{0}^{*}=w^{*}(x, 0) \text { e } & w_{0}=w(x, 0) \\
\left(\frac{\partial w^{*}}{\partial t}\right)_{0}=\frac{\partial w^{*}(x, 0)}{\partial} \text { e } & \left(\frac{\partial w}{\partial t}\right)_{0}=\frac{\partial w(x, 0)}{\partial}
\end{array}
$$

pode-se reescrever V.12, obtendo-se assim a equação integral transiente de placas. 


$$
\begin{aligned}
& \int_{\Omega}\left[w^{*}(x, t) f(x, t)-w(x, t) \delta(x) H(t)\right] d \Omega+\frac{1}{b^{2}} \int_{\Omega}\left[\frac{\partial w(x, 0)}{\partial} w^{*}(x, t)+w(x, 0) \frac{\partial w^{*}(x, t)}{\partial}-\frac{\partial w^{*}(x, 0)}{\partial} w(x, t)\right. \\
& \left.-w^{*}(x, 0) \frac{\partial w(x, t)}{\partial}\right] d \Omega=-\frac{1}{D} \int_{\Gamma}\left[V_{n}(w(x, t)) w^{*}(x, t)-M_{n}(w(x, t)) \frac{\partial w^{*}(x, t)}{\partial}+\frac{\partial w(x, t)}{\partial n} M_{n}\left(w^{*}(x, t)\right)\right. \\
& \left.-w(x, t) V_{n}\left(w^{*}(x, t)\right)\right] d \Gamma+\frac{1}{D} \sum_{i=1}^{N}\left\{\left[w(x, t) M_{n}\left(w^{*}(x, t)\right)-w^{*}(x, t) M_{n}(w(x, t))\right]_{i}\right\}
\end{aligned}
$$

\section{3 - Solução fundamental no domínio do tempo}

\subsection{1 - Equação diferencial transformada}

Sabe-se que a equação de movimento da placa (V.2) pode ser reescrita em coordenadas polares $(\boldsymbol{r}, \theta)$. No caso em que as vibrações da placa são simétricas em relação ao eixo $z$, o deslocamento fundamental $w^{*}$ e 0 carregamento fundamental $g^{*}$ são funçōes apenas de $r$ e $t$. Sendo assim, tém-se que:

$$
\frac{\partial()}{\partial \theta}=0
$$

e a equação V.2 passa a ser escrita como:

$$
b^{2}\left(\frac{\partial^{2}}{\partial r^{2}}+\frac{1}{r} \frac{\partial}{\partial r}\right)^{2} w^{*}+\frac{\partial^{2} w^{*}}{\partial^{2}}=\frac{g^{*}(r, t)}{2 \rho h}
$$

Sejam $w_{h}$ e $g_{h}$ as transformadas de Hankel de $w^{*}$ e $g^{*}$ tal que: 
100

$$
\begin{aligned}
& w_{h}(\xi, t)=\int_{0}^{\infty} r w^{*}(r, t) J_{0}(\xi r) d r \\
& g_{h}(\xi, t)=\int_{0}^{\infty} r g^{*}(r, t) J_{0}(\xi r) d r
\end{aligned}
$$

onde $J_{0}$ é a função de Bessel de ordem zero.

$O$ que se pretende é escrever a equação diferencial $V .16 \mathrm{em}$ função de $w_{h}$ e $g_{h}$. Para tanto considere a seguinte integração por partes:

$$
\begin{aligned}
\int_{0}^{\infty} r\left(\frac{\partial^{2} w^{*}}{\partial r^{2}}+\frac{1}{r}\right. & \left.\frac{\partial w^{*}}{\partial r}\right) J_{0}(\xi r) d r=\left[r \frac{\partial w^{*}}{\partial r} J_{0}(\xi r)\right]_{0}^{\infty}-\xi \int_{0}^{\infty} r \frac{\partial w^{*}}{\partial r} J_{0}^{\prime}(\xi r) d r \\
& +\int_{0}^{\infty} \frac{\partial w^{*}}{\partial r} J_{0}(\xi r) d r=\xi \int_{0}^{\infty} w^{*}\left\{J_{0}^{\prime}(\xi r)+\xi r J_{0}^{\prime \prime}(\xi r)\right\} d r
\end{aligned}
$$

sendo que $J_{0(\xi r)}$ satisfaz a seguinte equação diferencial

$$
\xi r J_{0}^{\prime \prime}(\xi r)+J_{0}^{\prime}(\xi r)+\xi r J_{0}(\xi r)=0
$$

Desta forma pode-se escrever que:

$$
\int_{0}^{\infty} r\left(\frac{\partial^{2} w^{*}}{\partial r^{2}}+\frac{1}{r} \frac{\partial w^{*}}{\partial r}\right) J_{0}(\xi r) d r=-\xi^{2} \bar{w}
$$


Aplicando-se duas vezes o operador, obtém-se:

$$
\int_{0} r\left(\frac{\partial^{2}}{\partial r^{2}}+\frac{1}{r} \frac{\partial}{\partial r}\right)^{2} w^{*} J_{0}(\xi r) d r=-\xi^{4} \bar{w}
$$

Então, multiplicando-se a equação $\mathrm{V} .16$ por $r J_{0(r \xi)}$ e integrandose com respeito a $\boldsymbol{r}$, cujos limites são $(0, \infty)$, pode-se escrevê-la em função de $w_{h}$. Desta forma a equação diferencial da placa passa e ser função apenas de t.

$$
\frac{d^{2} w_{h}}{d t^{2}}+b^{2} \xi^{4} w_{h}=(2 \rho h)^{-1} g_{h}(t \xi)
$$

Uma vez obtida $w_{h}$, através da solução da equação diferencial V.22, pode-se obter $w^{*}$ fazendo-se a inversa da transformada de Hankel V.17.

$$
w^{*}(r, t)=\int_{0}^{\infty} \xi w_{h}(\xi, t) J_{0}(\xi r) d \xi
$$

\subsection{2 - Solução da equação diferencial transformada}

Assumindo-se que o carregamento $g_{h}$ é aplicado sobre uma área circular da placa, de raio a, e que tem a seguinte magnitudade:

$$
g_{h}=16 \rho h b \delta(r) H(t)
$$

a equação diferencial V.22 assume a forma: 


$$
\frac{d^{2} w_{h}}{d t^{2}}+b^{2} \xi^{4} w_{h}=\frac{8 b}{\pi a^{2}} \delta_{h}(\xi) H(t)
$$

onde, no caso da força ser uniformemente distribuida sobre um círculo de raio a, pode-se escrever que:

$$
{ }_{n}(\xi)=\int_{0}^{a} r \delta(r) J_{0}(\xi r) d r=\frac{a J_{1}(a \xi)}{\xi}
$$

no caso da força ser concentrada, tém-se que $a \rightarrow 0$, e:

$$
\frac{2 J_{1}(a \xi)}{\xi}=\xi
$$

Resolvendo-se a equação diferencial V.22, após substituir-se V.27 e V.26, tém-se que:

$$
w_{h}=\frac{4}{\pi b}\left\lfloor\frac{1-\cos \left(b \xi^{2} t\right)}{\xi^{3}}\right]^{3} H(t)
$$

Fazendo-se a transformada inversa de Hankel obtém-se a solução fundamental $\boldsymbol{w}^{*}$.

$$
w^{*}=\frac{16 t}{\pi b}\left[\frac{\pi}{2}-S i(x)-\operatorname{sen} x+x C i(x)\right]
$$


onde $x=\frac{r^{2}}{4 b(t-\tau)}$ as funções Si e Ci são conhecidas como integrais de seno e cosseno, respectivamente, e estão definidas no início do trabalho.

A solução fundamental $w^{*}$ satisfaz a equação de movimento V.16 e é igual a zero para $t=0$, assim como a sua derivada em relação a $t$.

\section{4 - Equação integral escrita para os pontos de carregamento}

A equação V.14 está escrita em função da variável de espaço $x$, definida pelos pontos $\times 1$ e $\times 2$. Reescrevendo-a em funçāo dos pontos q (ponto de carregamento) e p (ponto qualquer do domínio), cuja distância entre si é igual a $r$, tém-se que:

$$
\begin{aligned}
& \int_{\Omega}\left[w^{*}(q, p, t) f(p, t)-w(p, t) \delta(q, p) H(t)\right] d \Omega+\frac{1}{b^{2}} \int_{\Omega}\left[\frac{\partial w(p, 0)}{\partial t} w^{\cdot}(q, p, t)+w(p, 0) \frac{\partial w^{*}(q, p, t)}{\partial t}\right] d \Omega \\
& =-\frac{1}{D} \int_{\Gamma}\left[V_{n}(p, t) w^{*}(q, p, t)-m_{n}(p, t) \frac{\partial w^{*}(q, p, t)}{\partial n}+\frac{\partial w(p, t)}{\partial n} m_{n} \cdot(q, p, t)-w(p, t) V_{n}^{*}(q, p, t)\right] d_{\Gamma} \\
& +\frac{1}{D} \sum_{i=1}^{N c}\left\{w(p, t) m_{n t} \cdot(q, p, t)-w^{*}(q, p, t) m_{n t}(p, t)\right]
\end{aligned}
$$

Para que a equação V.30, acima, seja escrita para os pontos de carregamento, basta resolver o segundo termo da primeira integral de domínio, correspondente ao carregamento fundamental, ou seja: 
$\int_{\Omega} w(p, t) \delta(q, p) H(t) d \Omega$

Já foi visto, no capítulo II, que para um ponto situado no domínio a integral V.31 se escreve como sendo:

$$
\int_{\Omega} w(p, t) \delta(q, p) H(t) d \Omega=w(q, t) H(t)
$$

No caso dos pontos fora do contorno sabe-se que a integral V.31 se anula. Uma vez feita a integral de domínio do carregamento fundamental, e levando-se em conta as propriedades da solução fundamental para $t=0$,

$$
\begin{aligned}
& w^{*}(q, p, 0)=0 \\
& \frac{\partial w^{*}(q, p, 0)}{\partial t}=0
\end{aligned}
$$

pode-se escrever a equação integral V.30 para um ponto $q$, do domínio.

$$
\begin{aligned}
& w(q, t) H(t)=\int_{\Omega} w^{*}(q, p, t) f(p, t) d \Omega+\frac{1}{b^{2}} \int_{\Omega}\left[\frac{\partial w(p, 0)}{\partial t} w^{\cdot}(q, p, t)+w(p, 0) \frac{\partial w^{*}(q, p, t)}{\partial t}\right] d \Omega \\
& \left.+\frac{1}{D} \int_{\Gamma}^{[} V_{n}(p, t) w^{\cdot}(q, p, t)-m_{n}(p, t) \frac{\partial w^{*}(q, p, t)}{\partial n}+\frac{\partial w(p, t)}{\partial n} m_{n} \cdot(q, p, t)-w(p, t) V_{n}^{*}(q, p, t)\right] d \Gamma \\
& -\frac{1}{D} \sum_{i=1}^{x_{c}}\left[w(p, t) m_{m} \cdot(q, p, t)-w^{\cdot}(q, p, t) m_{m t}(p, t)\right]
\end{aligned}
$$

No caso dos pontos fora do contorno o termo da esquerda da igualdade, na equação V.33, se anula. Nesse caso deve-se levar em conta uma 
translação temporal referente à distância que o load point guarda do contorno, e a velocidade da onda, conforme demonstrado em CODA \& VENTURINI (1995). Desta forma é possivel também aqui evitar os problemas de singularidade.

\section{5 - Integração no tempo}

\subsection{1 - Convolução das integrais}

Antes de fazer a integração espacial da equação V.33 e montar as matrizes que compoem o sistema, deve-se fazer a integração no tempo da mesma. Na integração no tempo, o periodo de tempo t é subdividido em intervalos de duração $\Delta t$.

Sejam duas funções $\psi$ e $\varphi$, funções de $w^{*}$ e $w$, respectivamente.:

$$
I(q, t)=\int_{\Gamma}\left[\varphi(w(p, t)) \psi\left(w^{\cdot}(q, p, t)\right)\right] \Gamma
$$

explicitando-se o produto de convolução destas funções, obtém-se:

$$
I(q, t)=\int_{\Gamma}\left[\int_{q_{1}}^{2} \psi\left(w^{*}(q, p, t-\tau)\right) p(w(p, t)) d \tau\right] d \Gamma
$$

Como apenas a função $\psi$ é integrável, em $V .35$, o produto de convolução pode ser escrito como: 


$$
I(q, t)=\int_{\Gamma} \varphi(w(p, t))\left[\int_{t 1}^{12} \psi\left(w^{*}(q, p, t-\tau)\right) d \tau\right] \Gamma
$$

Assim sendo, para a integração no tempo da equação integral V.33 apenas o que for função da solução fundamental deve ser integrado. A única exceção se dá para o caso do termo que contém a função Heaviside, nas equações integrais escritas para pontos do domínio. Neste caso tém-se que:

$$
\int_{t 1}^{12} w(q, t) H(t-\tau) d \tau=(t 2-t 1) w(q, t) H(t-\tau)=\Delta t . w(q, t) H(t-\tau)
$$

Para as outras integrações, das funções da solução fundamental, basta proceder à integração no tempo. Como exemplo, tém-se 0 resultado da integração no tempo de w'.

$$
\begin{aligned}
& \int_{t 1}^{12} w^{\cdot}(q, p, t-\tau) d \tau=\frac{1}{4 \pi}\left[\frac{b \pi(t-\tau)^{2}}{4}-\frac{b(t-\tau)^{2}}{2} \operatorname{Si}(x)+\frac{(t-\tau) r^{2}}{4} C i(x)\right. \\
& \left.-\frac{3 b(t-\tau)^{2}}{4} \operatorname{sen}(x)+\frac{(t-\tau) r^{2}}{16} \cos (x)+\frac{r^{4}}{64 b} \operatorname{Si}(x)\right]_{t 1}^{7^{2}} \\
& \text { onde: } \quad x=\frac{r^{2}}{4 b(t-\tau)}
\end{aligned}
$$

\subsection{2 - Intervalos de integração}

De acordo com as propriedades da função Heaviside $\mathrm{H}_{(t)}$ torna-se necessário definir os intervalos de integração que tornam a função diferente de 
zero. Sendo $\mathrm{C}_{1}$ a velocidade de propagação da onda na placa, determinada numericamente, tém-se que a distância temporal entre o ponto de carregamento e o ponto que está sendo integrado é igual a $r / C_{1}$. Assim sendo, a função Heaviside fica definida pelo intervalo:

$$
t-\tau-\frac{r}{C_{1}}
$$

Chamando-se de t1 e t2 os intervalos inferior e superior de integração, respectivamente, e de TA a diferença entre $t$ e $r / C_{1}$, definem-se três intervalos de integração:

$$
\begin{aligned}
& \text { para } t-t 2 \geq \frac{r}{C_{1}} \rightarrow \int_{1_{1}}^{2} \psi\left(w^{*}(q, p, t-\tau)\right) d \tau \\
& \text { para } t-t 1 \geq \frac{r}{C_{1}} \geq t-t 2 \rightarrow \int_{11}^{2 A} \psi\left(w^{*}(q, p, t-\tau)\right) d \tau \\
& \text { para } \frac{r}{C_{1}} \geq t-t 1 \rightarrow 0
\end{aligned}
$$

\section{6 - Montagem e resolução do sistema}

Uma vez efetuada a convolução no tempo da equação integral da placa, procede-se à integração do contorno e a determinação das matrizes que compoem o sistema. A forma de proceder à integraçāo e à montagem do sistema para cada passo de tempo é análoga à empregada no capítulo II. Isto 
significa que, para cada intervalo de tempo, será sempre escrito um sistema matricial do tipo:

$$
\underset{\sim}{H} \underset{\sim}{U}=\underset{\sim}{G} \underset{\sim}{P}+\underset{\sim}{F}
$$

Existe, contudo, uma diferença no comportamento do sistema V.40, que caracteriza o comportamento dinâmico. Para que se compreenda melhor este comportamento, apresenta-se a seguir o procedimento feito na resolução dos 2 primeiros passos de tempo.

Para o primeiro passo o sistema é calculado para o intervalo de tempo variando de 0 a $t_{1}$, sendo o tempo total de integração igual a $t_{1}$, e pode ser escrito da seguinte forma:

$$
\underset{\sim 11}{H} \underset{\sim 1}{U}=\underset{\sim 11}{G} \underset{\sim 1}{P}+\underset{\sim 1}{F}
$$

onde o primeiro índice, escrito nas matrizes $\boldsymbol{H}$ e $\mathbf{G}$, indica o passo em que está sendo gerado o sistema, e o segundo índice indica o número do intervalo de tempo correspondente. $O$ indice escrito nos vetores $\boldsymbol{U}, \boldsymbol{P}$ e $\boldsymbol{F}$ indica o passo em que está sendo gerado o sistema. Uma vez determinado os valores incógnitos para este primeiro passo, pode-se passar para a montagem do segundo.

No segundo passo de tempo o sistema é calculado em dois intervalos de tempo, de 0 a $t_{1}$ e de $t_{1}$ a $t_{2}$. Assim sendo, o sistema V.40 passa a ser escrito como se segue:

$$
\underset{\sim 21}{H} \underset{\sim 1}{U}+\underset{\sim}{H} \underset{22}{U} \sim_{2}=\underset{\sim 21}{G} \underset{\sim 1}{P}+\underset{\sim 22}{G} \underset{\sim 2}{P}+\underset{\sim 1}{F}+\underset{\sim 2}{F}
$$


Neste segundo passo o tempo total de integraçāo passa a ser $t_{2} e$ pode-se afirmar então que:

$$
\underset{\sim 22}{H}=\underset{\sim 11}{H} \quad \text { e } \quad \underset{\sim 22}{G}=\underset{\sim 11}{G}
$$

como $U_{1}, P_{1}$ e $F_{1}$ já são também conhecidos do passo anterior, pode-se resolver o sistema $V .42$ e obter os novos valores de $U_{2}$ e $P_{2}$. O mesmo raciocínio é feito para os passos que se seguem e assim os valores incógnitos são determinados em cada passo de tempo.

\section{7 - Exemplo numérico}

Para exemplificar o comportamento da solução fundamental apresentada, analisa-se uma placa circular simplesmente apoiada cujas constantes geométricas sào:

$$
\begin{aligned}
& E=10^{7} \\
& \nu=0.3 \\
& h=0.50 \\
& \rho=0.0002588 \\
& \text { raio }=5.0 \\
& q=300
\end{aligned}
$$

onde uma carga concentrada é aplicada no centro, constante no tempo. O comportamento do deslocamento transversal no ponto central está representado na figura 21. 


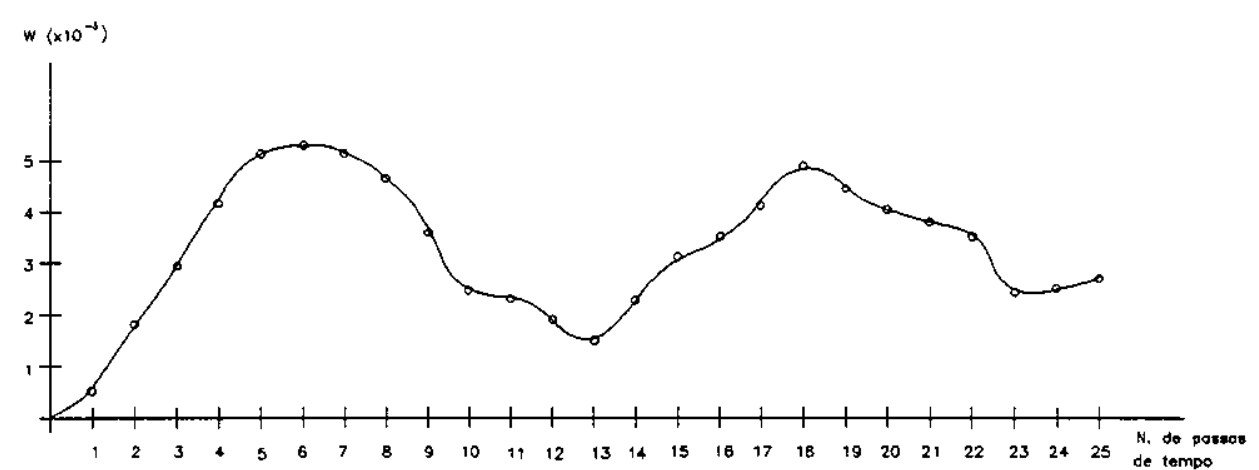

FIGURA 21

Como se pode observar, a partir da figura 21, a solução fundamental V.29 não apresenta comportamento estável ao longo da análise. Isso, provavelmente, se deve à imprecisão que ocorre na transformação da equação integral em equação algébrica. A onda que atravessa a placa é uma onda dissipativa e não se conhece a sua velocidade. Desta forma, a integração numérica que gera o sistema algébrico fica comprometida. $O$ fato dos pontos de carregamento terem sido colocados fora do contorno contribui para que esse problema de imprecisão aumente. 


\section{CAPÍTULO VI}

\section{CONCLUSÕES E CONSIDERAÇÕES FINAIS}

\section{1 - Formulação elastostática e elastodinâmica de placas}

Com relação à análise elastostática, a formulação adotada é a usualmente empregada quando se pretende tratar da análise de placas via BEM. Desta parte do trabalho dois aspectos merecem comentários, pelas vantagens que apresentaram. O primeiro aspecto é a alternativa de se adotar dois pontos fora do contorno para representar as equações dos nós que subdividem o contorno. Os resultados obtidos mostraram que esta forma de representar as equaçōes do contorno não acarreta em perda de acuidade e tem a grande vantagem de eliminar problemas de singularidade na integração dos elementos. $O$ outro aspecto diz respeito ao tratamento dado aos cantos da placa, onde escreveu-se uma equação independente para cada canto. Desta forma, a representação do canto deixa de influir na representação dos nós adjacentes ao mesmo.

Com relação à análise elastodinâmica o principal aspecto a ser enfatizado é o da versatilidade da formulação, que pode ser comprovada pela mínima perda da acuidade dos resultados quando se reduz a discretização do domínio. Tanto o contorno quanto o domínio podem ter uma discretização pobre que ainda assim obtém-se resultados satisfatórios, o que é uma grande vantagem 
quando se pensa em análises que envolvem um grande número de graus de liberdade, como é o caso da interação placa-solo. Neste aspecto, convém salientar também as vantagens do uso de células para calcular a integral de domínio do termo inercial. O uso da Quadratura de Gauss para resolver numericamente a integral de domínio mostrou-se muito eficiente e simplifica enormemente o cálculo. Por fim, um último aspeto a ser salientado diz respeito ao intervalo de tempo $(\Delta t)$ empregado. Este tipo de formulação elastodinâmica requer um $\Delta t$ grande para representar o problema (no caso do exemplo 00, utilizou-se um intervalo de tempo em torno de 3 vezes o comprimento do elemento).

\section{2 - Acoplamento placa-solo}

A formulação empregada na análise da interação placa-solo apresenta-se muito vantajosa em alguns aspectos. Um primeiro aspecto a ser resultado é a economia no número de variáveis necessárias para representar o problema. Uma outra vantagem está no fato da formulação representar de uma forma mais real as concentraçōes de tensão que ocorrem no contorno e, principalmente, nos cantos da placa, quando se trata de uma análise estática.

No caso da elastodinâmica, estas concentrações de tensão também ocorrem mas acabam por provocar perda de estabilidade da solução. Isto se deve ao fato da formulação conter um erro de aproximação na integração dos pontos onde existem essas concentrações, o que acarreta uma perda de representatividade quando de uma análise incremental. No caso do Método dos Elementos Finitos a aproximação empregada para integrar o contorno já amortece os efeitos das concentrações de tensão. Para se resolver este tipo de probelma poderia-se empregar um amortecimento que suavizasse os efeitos deste erro da integração, mas esta forma tem o incoviniente de amortecer todo o problema. 
Uma outra forma seria a de se adotar um modelo não linear para representar a região com concentrações de tensão, aliviando assim o erro da aproximação

Observando-se os resultados apresentados no primeiro exemplo, notam-se os efeitos provocados pelas diferentes formas de se analisar o acoplamento, principalmente entre os resultados obtidos por PAIVA quando comparados aos obtidos por CODA e por este trabalho. Neste caso os dois últimos, por terem o mesmo tipo de tratamento do solo, acabam apresentando resultados semelhantes, mostrando apenas as diferenças esperadas ao se analisar uma placa via FEM ou BEM. Porém, o tratamento que PAIVA dá ao solo e à placa, desconsiderando as forças de atrito e as outras componentes de deslocamento, acabam por provocar um deslocamento central maior.

A diferença na forma de tratar a placa, neste trabalho e em CODA, se torna mais visível e passa a representar um fator limitante ou não, quando se trata de uma análise dinâmica onde a rigidez do solo é bem diferente da rigidez da placa.

\section{3 - Solução fundamental transiente de placa, no domínio do tempo}

Uma outra forma adotada para se analisar a elastodinâmica de placas foi com o uso de uma solução fundamental transiente, no domínio do tempo. Contudo, como problemas de instabilidade surgiram e os esforços para superá-los não foram suficientes, acabou-se optando pela formulação alternativa, via matriz de massa, na análise da placa. Entretanto não se pretende abandonar a possibilidade de superar os problemas de instabilidade da solução. Um dos problemas detectados durante o trabalho com a solução foi com relação à imprecisão numérica que ela apresenta, devido às características trigonométricas da mesma, principalmente com a presença dos termos de integrais de seno e cosseno. Este tipo de problema em análise dinâmica acaba acarretando perda de 
estabilidade, no decorrer da análise, já que as diferenças acabam sendo acumuladas e levadas para o passo posterior.

Uma outra possibilidade de resolver os problemas de instabilidade da solução está em aplicar uma função que suavise o carregamento aplicado. Esta função deverá ter uma continuidade de ordem superior à empregada inicialmente, e assim as equações algébricas serão representações mais fiéis das equaçōes integrais. 


\section{BIBLIOGRAFIA}

ABRAMOWITZ, M. (1964). Handbook of mathematical functions with formulas, graphs and mathematical tables. Washington, National Bureau of Standards.

ANTES, H. (1988). Anwendung der Methode der Randelemente in der Elastodynamic und der Fluiddynamic. Inc: Mathematische Methoden in der Technik n. 9. Stuttgart, B. G. Teubner

ATLURI, S. N. ; GRANNEL, J. J. (1978). Boundary element methods (BEM) and combinations of BEM-FEM. Report n. GIT-ESM-SA-78-16.

BERGMAN, S. ; SCHIFFER, M. (1953). Functions and elliptic Differential equations in Mathematical Physics. Academic Press Inc, New York.

BESKOS, D. E. (1987). Mechanics and mathematical methods (v. 3). Amsterdam, North-Holland.

BETTI, E. (1872). Teoria dell elasticita. /l Nuovo Cimento, ser. 3, v. 610. 
BÉZINE, G. (1978). Boundary integral formulation for plate flexure with arbitrary boundary conditions. Mechanics Research Communications, v. 5, p. 197-206.

BÉZINE, G. (1981). A boundary integral equation method for plate flexure with conditions inside the domain. Int. J. for Numerical Methods in Engineering , v. 17, p. 1647-1657.

BÉZINE, G. (1981). Application of similarity to research of new boundary integral equations for plate flexure problems. Applied Mathematical Modelling, v. 5 , p. $66-70$.

BÉZINE, G. ; CIMETIERE,. A. ; GELBERT, J. P. (1985). Unilateral buckling of thin elastic plates by the boundary integral equation method. Int. J. for Numerical Methods in Engineering , v. 21 , p. 2189-2199.

BÉZINE, G. ; GAMBY, D. (1982). Étude des mouvements transitoires de flexion d'une plaque par la méthode des équations intégrales de frontière. Journal de Mécanique Appliquée, v.1, n.3, p.451-466.

BRADY, B. H. G. ; WASSYNG, A. (1981). A coupled finite element boundary element method of stress analysis. Int. J. Rock Mech. Min. Sciences, v. 18 , p. $475-485$.

BREBBIA, C. A. ; TELLES, J. C. F. ; WROBEL, L. C. (1984). Boundary element techniques: Theory and applications engineering. Berlin, Springer Veralg. 
BREBBIA, C. A. ; FERRANTE, A. J. (1975). The finite element technique. Porto Alegre, Ediçōes URGS.

BRONSTEI, I. ; SEMENDJAJEW, K. (1979). Taschenbuch der Mathematik (neubearbeitung). Harri Deutsch Verlag.

BOUSSINESQ, M. J. (1885). Application des potentiels : a l'etude de l'equilibre et du mouvement des solides elastiques. Paris, Gauthier-Villars.

CARRER, J. A. M. (1991). Técnicas implicitas para análise elastoplástica estática e dinâmica com o método dos elementos de contorno. Rio de Janeiro. Tese (doutorado) - COPPE, UFRJ.

CAUCHY, A. L. (1828). Exercices de mathématique, sur l'équilibre et le mouvement d'un systemé de points matériels par des forces d'attraction ou de répulsion mutuelle. apud: LOVE, A. E. H. (1944). A treatise on the mathematical theory of elasticity. New York, Dover.

CHOI, C. K. ; KIM, H. S. (1993). Variable node plate bending element for mat foundation analysis. Computers and Structures, v. 47, n. 3, p. $371-$ 381.

CLOUGH, R. W. ; FELIPPA, C. A. (1968). A refined quadrilateral element for analysis of plate bending. In: CONFERENCE ON MATRIX METHODS IN STRUCTURAL MECHANICS, 2 ..

CODA, H. B. (1993). Análise tridimensional transiente de estruturas pela combinação entre o método dos elementos de contorno e o método dos 
elementos finitos. São Carlos. Dissertação (mestrado) - Escola de Engenharia de São Carlos, Universidade de São Carlos.

CODA, H. B. et VENTURINI, W. S. (1990). Alternative boundary element formulation for elastodynamics. In: INTERNATIONAL CONFERENCE ON BOUNDARY ELEMENTS IN ENGINEERING, 12., Sapporo, Japan, 24-27 Sept., 1990. Proceedings. Southampton, CML; Berlin, Springer-Verlag, v. 1 , p. $517-534$.

CODA, H. B. ; VENTURINI, W. S. (1995). Non-singular time-stepping BEM for transient elastodynamic analysis. Engineering Analysis with Boundary Elements, v. 15, p. 11-18.

CODA, H. B. ; VENTURINI, W. S. (1995). Three-dimensional transient BEM analysis. Computers \& Structures, v. 56, n. 5, p. 751-768.

CRUSE, T. A. et RIZZO, F. J. (1968). A direct formulation and numerical solution of the general transient elastodynamic problem. Int. J. Mathematical Analytical Applied, v. 22, p. 244-259.

CRUSE, T. A. (1968). A direct formulation and numerical solution of the general transient elastodynamic problem, II. int. J. Mathematical Analytical Applied, v. 22, p. 341-355.

DALE, J. B. (1903). Five-figure tables of mathematical functions. London, E. Arnold. 
DANSON, D. J. (1979). Analysis of plate bending problems by direct boundary element method. Southampton. M. Sc. Dissertation University of Southampton.

DAVIS, P. J. ; RABINOWITZ, P. (1984). Methods of numerical integration. San Diego, Academic Press.

ERINGEN, A. C. ; SUHUBI, E. S. (1974). Elastodynamics: Linear Theory, vol. 2. Academic Press, New York.

ESTORFF, O. von (1992). Coupling of BEM and FEM in the time domain: some remarks on its applicability and efficiency. Computers and Structures , v. 44 , n. $1 / 2$, p. $325-337$.

GIL RODRIGUES, J. C. (1986). Sobre o emprego do método dos elementos de contorno em problemas elásticos bidimensionais. São Carlos. Dissertação (mestrado) - Escola de Engenharia de São Carlos, Universidade de São Paulo.

GANGMING, L. (1989). A new boundary element method coupled with FEM packages. Communications in Applied Numerical Methods, v.5, p. 365371.

GROBNER, W. ; HOFREITER, N. (1961). Integraltafel (erster Teil) Unbestimmte Integrale. Berlin, Springer Verlag.

HELL, M. E. (1986). Linguagem de programação estruturada FORTRAN 77. São Paulo, McGraw-Hill. 
HINTON, E. (1988). Numerical methods and software for dynamic analysis of plates and shells. Swansea, Pineridge Press.

JASWON, M. A. et alii (1967). Numerical biharmonic analysis and some applications. Int. J. Solids Structures, v. 3, p.309-332.

KIRCHHOFF, G. (1850). Über das Gleichgewicht und die Bewegung einer elastichen Scheibe. J. Mathematics. v. 40, p. 51-58.

KITAHARA, M. (1985). Boundary integral equation methods in eigenvalue problems of elastodynamics and thin plates. Amsterdan, Elsevier.

KUPRADZE, V. D. (1965). Potencial methods in theory of elasticity. Jerusalem, Israel Program for Scientific Translation.

KUTT, H. R. (1975). WISK 178: quadrature formulae for finite-part integrals. Pretoria, National Research Institute for Mathematical Sciences. CSIR Special Report.

LACHAT, J. C. (1975a). A further development of the boundary integral technique for elastostatics. Ph.D. Thesis, University of Southampton.

LACHAT, J. C. ; WATSON, J. O. A. (1975a). A second generation boundary integral equation program for three dimensional elastic analysis. In: CRUSE, T. A. ; RIZZO, F. J., eds. Boundary integral equation method: computational applications in applied mechanics. ASME, p.85100. 
LEOFFLER NETO, C. F. (1988). Uma formulação alternativa do método dos elementos de contorno aplicada a problemas de campo escalar. Rio de Janeiro. Tese (doutorado) - Universidade Federal do Rio de Janeiro.

LOVE, A. E. H. (1944). A treatise on the mathematical theory of elasticity. New York, Dover.

LOWAN, A. N. (1940). Tables of sine, cosine and exponential integrals. New York, National Bureau of Standards.

MANSUR, W. J. ; BREBBIA, C. A. (1985). Transient Elastodynamics. In: Topics in Boundary Element Research - 2. Berlin, Springer Verlag.

MESSAFER, T. ; COATES, L. E. (1989). An Application of FEM/BEM coupling to foundation analysis. Inc: International Conference on Boundary Element Methods , 11., Cambridge, USA. Proceedings . v. 3, p. 211-223.

MITSUI, Y. et alii. (1985). A coupling scheme for boundary and finite elements using a joint element, Int. J. for Numerical and Analytical Methods in Geomechanics, v. 9, p. 161-172.

NARDINI, D. ; BREBBIA, C. A. (1982). A new approach to free vibration analysis using boundary elements. Boundary Element Method in Engineering. Berlin, Springer-Verlag.

NARDINI, D. ; BREBBIA, C. A. (1983). Dynamic analysis in solid mechanics by alternative boundary element procedure. Int. J. Soil Mechanics and Earthquake Engineering , v. 2, p. 228-233. 
NAVIER (1827). Mém. Acad. Sciences. Paris, v. 7. apud LOVE, A. E. H. (1944). A treatise on the mathematical theory of elasticity. New York, Dover.

OLIVEIRA NETO, L. (1991). Análise de placas de borda curva pelo método dos elementos de contorno. São Carlos. Dissertação (mestrado) Escola de Engenharia de São Carlos, Universidade de São Paulo.

PAIVA, J. B. (1990). Formulação do método dos elementos de contorno para flexão de placas e suas aplicações em engenharia de estruturas. São Carlos. Tese (doutorado) - Escola de Engenharia de São Carlos, Universidade de São Paulo.

PAIVA, J. B. ; BUTTERFIELD, R. (1994). Numerical analysis of plate-soil interaction. In: PAPADRAKAKIS, $M$. et TOPPING, B. H. V., eds. Advances in computational mechanics. (Proc. 2nd international Conference on Computational Structures Technology, Athens, Greece, 30th Aug. - 1st Sept.). Edinburgh, Civil-Comp Press. p. 275-281.

REDDY, J. N. (1993). An introduction to the finite element method. New York, McGraw-Hill.

REY PASTOR, J. ; BRZEZICKI, A. C. (1958). Funciones de Bessel. Madrid, Editorial Dossat, S.A.

RIZZO, F. J. (1967). An integral equation approach to boundary value problems of classical elastostatics. Quarterly Applied Mathematic , v. 25 , p. 83-95. 
RUNGE, C. (1908). Zeit. Math. Phys. , v. 56, p. 225.

SNEDDON, I. N. (1945). The symmetrical vibration of a thin elastic plate. Proceeding Cambridge Philosophical Society, v. 41, n. 1, p.27-43.

SOUTHWELL, R. V. (1946). Relaxation methods in theoretical physics. London, Oxford University Press.

SPIEGEL, M. R. (1979). Manual de fórmulas e tabelas matemáticas. São Paulo, McGraw-Hill.

STERN, M. (1979). A general boundary integral formulation for the numerical solution of plate bending problems. Int. J. Solids Structures, v. 15 , p. $769-782$.

STROUD, A. H. ; SECREST, D. (1966). Gaussian quadrature formulas. New York, Prentice-Hall.

SZILARD, R. (1974). Theory and analysis of plates: classical and numerical methods. New Jersey, Prentice-Hall.

TEJERINA CALDERÓN, E. (1991). Uma formulação alternativa para o estudo de placas sobre fundação elástica pelo método dos elementos de contorno. São Carlos. Dissertação (mestrado) - Escola de Engenharia de São Carios, Universidade de São Paulo.

THOMSON, W. ; TAIT, P. G. (1962). Treatise on Natural Philosophy (reprinted as Principles of Mechanics and Dynamics). Dover, New York. 
TIMOSHENKO, S. P. ; WOINOWSKY-KRIEGER, S. (1970). Teoria de placas y laminas. Madrid, Ediciones Urmo.

TIMOSHENKO, S. P. ; GOODIER, J. N. (1980). Teoria da Elasticidade. Rio de Janeiro, Guanabara Dois.

VENTURINI, W. S. (1983). Boundary element method in geomechanics. Berlin, Springer-Verlag.

WARBURTON, G. B. (1964). The dynamical behavior of structures. New York, Pergamon Press.

WHEELER, L. T. ; STERNBERG, E. (1968). Some theorems in classical elastodynamics. Archive for Rational Mechanics and Analysis, v. 31, n. 1, p.51-90.

ZIENKIEWICZ, O. C. ; CHEUNG, Y. K. (1967). The finite element method in structural and continuum mechanics. London, McGraw-Hill.

ZIENKIEWICZ, O. C. ; CHEUNG, Y. K. (1965). Plates and tanks on elastic foundations - an application of finite element method. Int. J. Solids Structures, v. 1, p. 451-461.

ZIENKIEWICZ, O. C. et alii (1977). The coupling of the finite element method and boundary solution procedures. Int. J. Numerical Methods in Engineering, v. 11, p. 355-375. 


\section{APÊNDICE A}

\section{A.1 Integração numérica via Quadratura de Gauss}

A integração numérica é uma forma de se resolver uma integral quando a solução analítica desta se mostra difícil. Ela consiste em transformar um problema contínuo, que seria o cálculo da integral, em um problema discreto. $O$ incoveniente desta transformação é que ela resulta, na maior parte dos casos, num resultado aproximado, diferente do resultado exato. É possível forçar a que o resultado aproximado convirja em direção do valor exato, mas isto, em geral, causa um aumento no trabalho numérico. A precisão a ser obtida vai depender de cada problema a ser analisado.

Existem diversas maneiras de se calcular numericamente uma integral. No presente trabalho optou-se pelo método da Quadratura de Gauss. Neste método a transformação da integral se dá da seguinte forma:

$$
\int_{-1}^{1} f_{(\xi)} d \xi \cong \sum_{i=1}^{n} A_{i} f_{\left(\xi_{l}\right)}
$$

onde $\boldsymbol{n}$ é o número de pontos de Gauss a serem empregados na integração, $\boldsymbol{x}_{\boldsymbol{i}}$ são os pontos onde a função é calculada, e $\boldsymbol{A}_{i}$ são os pesos correspondentes aos pontos de Gauss.

Para se reduzir o erro, relativo ao valor exato, a primeira opção é aumentar o número de pontos de Gauss. Entretanto, para certas funçōes, é mais conveniente subdividir 0 intervalo de integração em subintervalos, ou subelementos. 


\section{A.2 - Integração sobre o elemento de contorno}

Generalizando a integração A.1, para quando a integração se dá em um intervalo genérico [a,b],

$$
\int_{a}^{b} g_{(x)} d x
$$

deve-se proceder da seguinte maneira: primeiramente associa-se a cada ponto ponto $x_{i}$, do intervalo $[\mathbf{a}, \mathbf{b}]$, um ponto $\xi_{i}$ do intervalo $[-1,1]$, através da relaçäo linear

$$
x_{i}=\frac{b+a}{2}+\frac{b-a}{2} \xi_{i}
$$

Em seguida deve-se relacionar os diferenciais $\boldsymbol{d}_{\boldsymbol{x}}$ e $\boldsymbol{d}_{\xi}$. Essa relação entre os diferenciais é chamada de Jacobiano e indicada por $J$. No caso:

$$
J=\frac{d x}{d \xi}=\frac{b-a}{2}
$$

Assim sendo, a integral A.2 pode ser resolvida numericamente como se segue:

$$
\int_{a}^{\beta} g_{(x)} d x=\frac{b-a}{2} \sum_{i=1}^{n} A_{i} f_{\left(x_{t}\right)}
$$

No caso das integrais sobre os elementos, observa-se que o ponto a ser integrado, e que pertence ao contorno, é o ponto $\boldsymbol{p}$. Para facilitar o acompanhamento das relações a serem feitas, deve-se observar a figura (22), onde está representado um elemento quadrilátero, de nós 1, 2 e 3. 


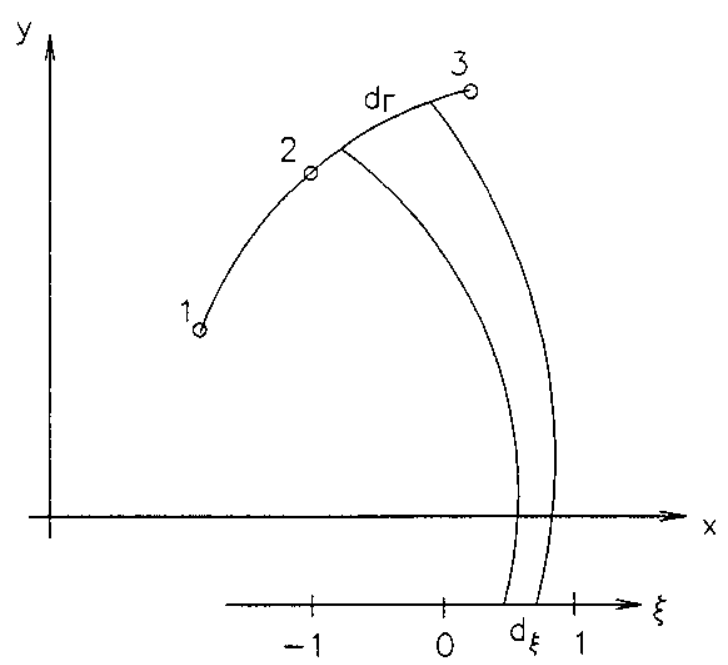

FIGURA 22

O ponto $\boldsymbol{p}$ pertence a este elemento e tem as coordenadas $x_{p}$ e $\boldsymbol{y}_{\boldsymbol{p}}$ definidas pelas relações

$$
\begin{aligned}
& x_{p_{t}}=\frac{x_{3}+x_{1}}{2}+\frac{x_{3}-x_{1}}{2} \xi_{i} \\
& y_{p_{t}}=\frac{y_{3}+y_{1}}{2}+\frac{y_{3}-y_{1}}{2} \xi_{i}
\end{aligned}
$$

O jacobiano desta integral é definido como sendo:

$$
J=\sqrt{\left(\frac{d x}{d \xi}\right)^{2}+\left(\frac{d y}{d \xi}\right)^{2}}
$$

Definidas as relações e tomando-se como exemplo a primeira das integrais da equação 11.69 , pode-se calculá-la numericamente com: 


$$
\int_{\Gamma} \phi_{1(p)} V_{n(q, p)}^{*} d_{\Gamma(p)}=\int_{-1}^{\Gamma} J_{1_{(p)}} \phi_{1(p)} V_{n(q, p)}^{*} d_{\xi_{(p)}}
$$

\section{A.3 - Integração no domínio}

Observando a figura (23) abaixo,

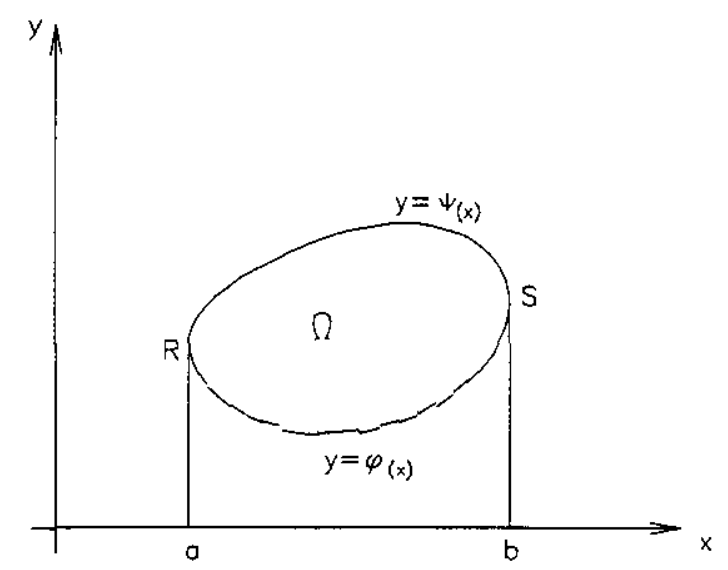

FIGURA 23

vê-se claramente que a integral da função $f_{(x, y)}$ no domínio $\Omega$ nada mais é que uma integral dupla, ou seja:

$$
\int_{\Omega} f_{(x, y)} d_{\Omega}=\int_{a}^{\beta}\left(\int_{\varphi_{(x)}}^{\psi_{(x)}} f_{(x, y)} d y\right) d x
$$

A integral interna de A.9 é uma função de $x$, e pode ser chamada de $g_{(x)}$. Assim A.9 fica escrita como se segue: 


$$
\int_{\Omega} f(x, y) d_{\Omega}=\int_{a}^{b} g_{(x)} d x
$$

A intgeral fica em função de uma única variável e sua solução numérica já se conhece do ítem anterior e é da forma:

$$
\int_{a}^{b} g_{(x)} d x=\frac{b-a}{2} \sum_{i=1}^{n} A_{i} g_{\left(x_{i}\right)}
$$

Por sua vez, a integral interna de A.9 passa também a ser função de uma única variável e pode ser resolvida da mesma forma.

$$
g_{\left(x_{1}\right)}=\int_{\varphi_{\left(x_{i}\right)}}^{\psi_{\left(x_{i}\right)}} f_{\left(x_{i}, y\right)} d y=\frac{\psi_{\left(x_{i}\right)}-\varphi_{\left(x_{i}\right)}}{2} \sum_{j=1}^{m} A_{j} f_{\left(x_{1}, y_{j}\right)}
$$

A combinação das duas integrações leva, finalmente à solução numérica da integral no domínio A.9

$$
\int_{\Omega} f_{(x, y)} d_{\Omega}=\frac{b-a}{2} \frac{\psi_{\left(x_{i}\right)}-\varphi_{\left(x_{i}\right)}}{2} \sum_{i=1}^{n} \sum_{j=1}^{m} A_{i} A_{j} f_{\left(x_{i}, y_{j}\right)}
$$

\section{A.4 - Jacobiano da célula quadrilátera}

As funções de interpolação da célula quadrilátera adotada neste trabalho estão escritas no capítulo III. Como se sabe, pode-se determinar as coordenadas de um ponto qualquer da célula a partir das funções interpoladoras, através das relações: 
$x=\sum_{i=1}^{8} \phi_{i} x^{i} ; y=\sum_{i=1}^{8} \phi_{i} y^{i} ; z=\sum_{i=1}^{8} \phi_{i} z^{i}$

O jacobiano é calculado através da expressão:

$$
|J|=\sqrt{g_{1}^{2}+g_{2}^{2}+g_{3}^{2}}
$$

onde:

$$
\begin{aligned}
& g_{1}=\frac{\partial y}{\partial \xi} \frac{\partial z}{\partial \eta}-\frac{\partial y}{\partial \eta} \frac{\partial z}{\partial \xi} \\
& g_{2}=\frac{\partial z}{\partial \xi} \frac{\partial x}{\partial \eta}-\frac{\partial x}{\partial \xi} \frac{\partial z}{\partial \eta} \\
& g_{3}=\frac{\partial x}{\partial \xi} \frac{\partial y}{\partial \eta}-\frac{\partial y}{\partial \xi} \frac{\partial x}{\partial \eta}
\end{aligned}
$$

